\title{
MOTIONS OF IONS AND ELECTRONS
}

W. P. ALLIS

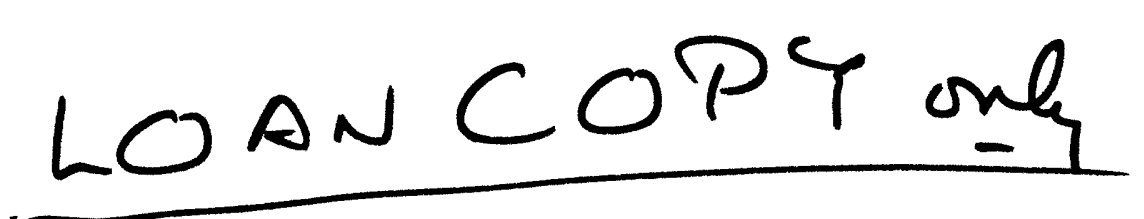

TECHNICAL REPORT 299

JUNE 13, 1956

RESEARCH LABORATORY OF ELECTRONICS MASSACHUSETTS INSTITUTE OF TECHNOLOGY

CAMBRIDGE, MASSACHUSETTS 
The Research Laboratory of Electronics is an interdepartmental laboratory of the Department of Electrical Engineering and the Department of Physics.

The research reported in this document was made possible in part by support extended the Massachusetts Institute of Technology, Research Laboratory of Electronics, jointly by the U. S. Army (Signal Corps), the U. S. Navy (Office of Naval Research), and the U. S. Air Force (Office of Scientific Research, Air Research and Development Command), under Signal Corps Contract DA36-039-sc-64637, Project 102B; Department of the Army Projects 3-99-10-022 and DA3-99-10-000. 


\title{
MASSACHUSETTS INSTITUTE OF TECHNOLOGY \\ RESEARCH LABORATORY OF ELECTRONICS
}

Technical Report 299

June 13, 1956

\section{MOTIONS OF IONS AND ELECTRONS}

W. P. Allis

Most of this report is identical with material prepared for Handbuch der Physik, Volume XXI, 1956.

\begin{abstract}
This report reviews the mathematical methods applicable to ionized gases. In Part I the motion of an individual electron or ion under a Lorentz force, including the effects of magnetic gradients, is studied. In Part II, with the introduction of collisions, this is no longer possible, but we can still follow the motion of an average particle. For high values of $\mathrm{E} / \mathrm{p}$, the behavior of a swarm of particles is remarkably close to the motion of the average particle, but for lower values of $\mathrm{E} / \mathrm{p}$ other methods must be used. In Part III the Boltzmann equation is applied to a "Lorentzian gas," that is, to free electrons in a gas. In Part IV the equation is transformed to an integral form, the Boltzmann trans port equation, so that it will be applicable to ions. The transport equation is solved exactly for the case of particles with a constant mean free time, which corresponds to the polarization force between an ion and a molecule. The consideration of Coulomb interactions in Part $\mathrm{V}$ introduces a force of so much longer range that an electron interacts simultaneously with a large number $\mathrm{N}$ of electrons and ions. The basic assumption of Boltzmann theory is then violated, and one must use the Fokker-Planck equation, the relation of which to the Boltzmann transport equation is shown. The coefficients of the Fokker-Planck equation are derived by the method of the Rosenbluth potentials.
\end{abstract}



Introduction 1

I. Electron and Ion Orbits 2

a. Orbits in uniform fields 2

1. Orbits in a uniform magnetic field only 2

2. Orbits in uniform electric and magnetic fields 3

3. Orbits in a uniform magnetic field and alternating electric field 4

4. Orbits in a changing magnetic field 6

b. Orbits in inhomogeneous magnetic fields $r$

5. Longitudinal magnetic gradient $\quad 7$

6. Transverse gradients $\quad 8$

7. The drift velocity $r$

c. Pressure gradients 11

8. Equation for the drift velocity 11

9. Equilibrium 12

10. Stationary distributions 13

a. Straight parallel magnetic field lines 13

$\beta$. Circular magnetic field lines $\quad 13$

II. The Langevin Equation $\quad 15$

a. Mobility 15

11. Relaxation times 15

12. Mobility 16

b. Diffusion 19

13. The random flight 19

14. The Einstein relation $\quad 21$

15. Ambipolar diffusion $\quad 22$

c. Energy and gain $\quad 26$

16. Elastic collisions only 26

17. Energy gain and inelastic collisions $\quad 28$

III. The Boltzmann Equation $\quad 32$

a. The derivative terms

18. Continuity in phase space 32

19. Expansion in spherical harmonics $\quad 32$

20. The gradient term 34

21. Electric terms $\quad 35$

22. Magnetic terms $\quad 35$

23. Expansion in Fourier series $\quad 36$

24. The component equations $\quad 36$

b. The collision integral $\quad 38$

25. Geometry of a collision 38

26. Collision integrals $\quad 40$

27. Expansion in spherical harmonics 41

28. Recoil $\quad 42$

29. Inelastic collisions $\quad \mathbf{4 4}$

c. The drift motion $\quad 45$

30. Diffusion $\quad 45$

31. Mobility 46 
d. The energy distribution

32. Plasma balance

33. The second-order equation 49

34. The gain equation 51

35. The distribution function

e. Direct current $\quad 57$

36. The first Townsend coefficient $\quad 57$

IV. Boltzmann Transport Equation $\quad 60$

a. General theory $\quad 60$

37. Transport equation $\quad 60$

38. Recursion equations $\quad 62$

b. Constant mean free time $\quad 64$

39. Drift velocity $\quad 64$

40. Energy $\quad 65$

$\begin{array}{ll}\text { c. Constant mean free path } & 67\end{array}$

41. Heavy ions $\quad 67$

42. Light ions $\quad 68$

d. Polarizable molecules $\quad 70$

43. Force between ion and molecule $\quad 70$

44. The Langevin theory 12

V. The Fokker -Planck Equation $\quad 75$

a. Flow in velocity space $\quad 75$

45. Relation of the Fokker-Planck equation to the transport equation $\quad 75$

$\begin{array}{ll}\text { 46. Conservation theorems } & 77\end{array}$

b. Rutherford scattering $\quad 77$

47. Mean deflections $\quad 77$

48. Shielded Rutherford scattering $\quad 79$

49. Rosenbluth potentials for a single scattering $\quad 82$

50. Coulomb's law in velocity space $\quad 83$

51. The potentials of a scattering field $\quad 85$

52. Flow in velocity space $\quad 87$

c. Maxwellian distribution of scatterers $\quad 88$

53. Reduction of the flow vector $\quad 88$

54. Electron interactions 9

55. Electron-ion interactions 92

56. Conductivity of a partially ionized plasma 93

d. Stochastic processes $\quad 94$

57. The Fokker-Planck equation $\quad 94$

58. The Langevin equation 95

$\begin{array}{ll}\text { References } & 98\end{array}$

$\begin{array}{lr}\text { Bibliography } & 100\end{array}$ 


\section{Introduction}

This report is divided into five parts according to the mathematical method used, rather than according to the physical situation. In Part I we attempt to follow the motion of an individual particle under a Lorentz force, including the effects of gradients of the magnetic field. This is no longer possible with the introduction of collisions in Part II, but one may yet follow the motion of an average particle. One is then tempted to expect that a swarm of particles will have the behavior of the average particle, and this is remarkably close to the truth for high values of $E / p$, the ratio of the continuous to the stochastic force, and has the advantage of being more intuitive than the distribution function methods which follow.

The method of orbits can be extended to give dispersion about the mean but this is unprofitable. If one cannot follow an individual particle experimentally it is best to let it lose its identity in the theory as well, and calculate the distribution function $f(\vec{r}, \vec{v}, t)$ of an assembly. The Boltzmann equation then replaces the Newtonian equations.

In Part III the Boltzmann equation is applied to a "Lorentzian gas," a gas composed of light particles which do not collide with each other, but do collide with heavier particles; that is to free electrons in a gas.

The Boltzmann equation is difficult to solve when the restriction to light particles is removed, in Part IV, so that it will be applicable to ions. The equation is then transformed to an integral form, the Boltzmann transport equation, which gives directly the average value of any quantity $X(v)$ depending on the velocity of the ions. The transport equation contains the unknown $f(v)$, but as it is under an integral sign an approximation to $f$ results in a higher approximation for $\bar{X}$. This property of the transport equation was shown by Maxwell to be particularly prominent if the law of force between particles varied inversely as the fifth power of their distance. In that case the equation for $\overline{\mathrm{X}}$ is completely independent of $f$. Fortunately the inverse fifth power law is exactly right for a slow ion and a molecule, aside from the property of charge transfer.

The consideration of Coulomb interactions in Part V introduces a force of so much longer range that an electron is interacting simultaneously with many others, and the number $\mathrm{N}$ with which it interacts simultaneously enters explicitly into the equations. The basic assumption of Boltzmann theory is that the interaction time $\Delta t$ is very small. With Coulomb interactions the fluctuation time $\boldsymbol{T}$ is smaller than $\Delta t$, and the appropriate equation for this situation is that of Fokker-Planck. 
When the interactions between electrons are large compared with their interactions with the gas, a new type of phenomenon appears: collective oscillations superposed on the random motions. These will not be considered here, partly because the subject is new and not well understood.

MKS rationalized units will be used throughout this report. In this system the permeability of free space is $\mu_{0}=4 \pi \times 10^{-7}$ henry/meter and the permittivity is $\epsilon_{\mathrm{o}}=1 / \mu_{\mathrm{o}} \mathrm{c}^{2}$ farad/meter. To convert to cgs units, substitute $4 \pi \epsilon_{\mathrm{o}}=1 . \mathrm{q}= \pm Z$ e is the charge on the ion, and $\mathrm{e}=1.602 \times 10^{-19}$ coulombs is always positive. The word "ion" is generally used to distinguish the heavy particle from an electron, but it is also occasionally used to mean either ion or electron, as in the preceding sentence, care being taken in the context so that it is not misunderstood.

\section{ELECTRON AND ION ORBITS}

\section{a. Orbits in Uniform Fields}

1. Orbits in a uniform magnetic field only. In the absence of collisions, a particle of charge $q$ moving in a magnetic field $\vec{B}$ is subject to a force

$$
\overrightarrow{\mathrm{F}}=\mathrm{q} \overrightarrow{\mathrm{v}} \times \overrightarrow{\mathrm{B}}=\mathrm{mv}
$$

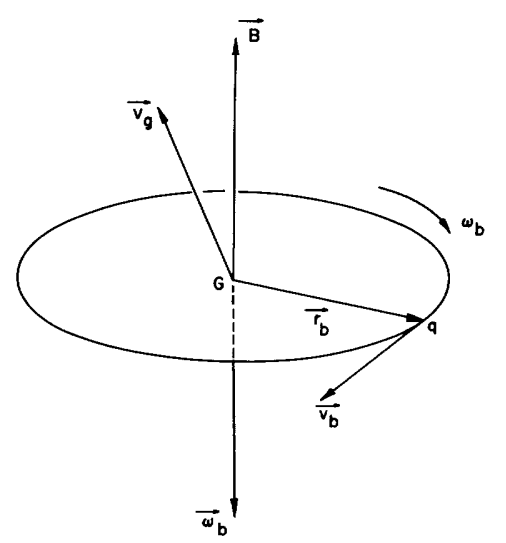

Fig. 1. Vector relations in the motion of an ion in a magnetic field.
We shall assume that the motion of the particle is the resultant of a circular motion $\vec{v}_{b}$ about a guiding center $G$, which itself has a uniform motion $\vec{v}_{g}$

$$
\vec{v}=\vec{v}_{g}+\vec{v}_{b}=\vec{v}_{g}+\vec{\omega}_{b} \times \vec{r}_{b}
$$

The acceleration for this motion is

$$
\dot{\vec{v}}=\vec{\omega}_{b} \times \vec{v}_{b}=-\omega_{b}^{2} \vec{r}_{b}
$$

Substituting in Eq. 1.1 gives

$$
q \vec{v}_{g} \times \vec{B}+q \vec{v}_{b} \times \vec{B}=m \vec{\omega}_{b} \times \vec{v}_{b}
$$

whence

$$
\begin{aligned}
& \vec{\omega}_{b}=-q \vec{B} / m \\
& \vec{v}_{g} \times \vec{B}=0
\end{aligned}
$$


Thus the motion is circular around $\overrightarrow{\mathrm{B}}$ with the "cyclotron" frequency $\nu_{\mathrm{b}}$ given by

$$
\begin{aligned}
\frac{\nu_{\mathrm{b} 1}}{\mathrm{~B}}=\frac{\mathrm{q}}{2 \pi \mathrm{m}} & =2.799 \mathrm{mc} / \text { gauss for electrons } \\
& =1.525 \mathrm{kc} / \text { gauss for protons }
\end{aligned}
$$

combined with an arbitrary uniform translation along $\vec{B}$. Equation 1.4 will be kept as the definition of the cyclotron vector $\vec{\omega}_{\mathrm{b}}$. Equation 1.5 will be generalized in the following sections.

2. Orbits in uniform electric and magnetic fields. A particle in the presence of both an electric and a magnetic field experiences the Lorentz force

$$
\vec{F}=q(\vec{E}+\vec{v} \times \vec{B})=m \dot{\vec{v}}
$$

If we assume a solution of this equation of the form Eq. 1.2, we find that

$$
\vec{E}+\vec{v}_{g} \times \vec{B}=0
$$

which yields

$$
\vec{v}_{g}=\vec{E} \times \vec{B} / B^{2}+\vec{v}_{\|}
$$

where the velocity $\vec{v}_{\|}$parallel to the magnetic field is not determined by Eq. 2.2 but satisfies the usual scalar equation

$$
\mathrm{m} \dot{\mathrm{v}}_{\|}=\mathrm{qE} \|
$$

hence the conservation of energy principle

$$
\frac{1}{2} m v_{\|}^{2}+q V=\text { constant }
$$

The motion of the guiding center is therefore normally accelerated along the magnetic field and has a constant drift of magnitude $E_{\perp} / B$ across the magnetic field. If the medium is neutral, containing equal amounts of positive and negative charges, an electric field produces motion along $\vec{B}$ in the opposite direction for the two signs of charge; hence a net electric current along the field. But the cross drift is the same for both kinds of charge; hence in this direction $\vec{E}$ produces a mass motion with no electric current. 
3. Orbits in a uniform magnetic field and alternating electric field. If an alternating electric field

$$
\vec{E}=\vec{E}_{p} e^{j \omega t}
$$

is superposed on a magnetic field $\vec{B}$, the resultant velocity will have a random component with three arbitrary constants

$$
\vec{v}_{r}=\vec{v}_{\| l}+\vec{w}_{b} \times \vec{r}_{b}
$$

in which $\vec{v}_{\|}$is constant (zero frequency) and $\vec{\omega}_{b} \times \vec{r}_{b}$ has the cyclotron frequency, plus a forced motion, $v_{g} e^{j \omega t}$, at the applied frequency, which has no arbitrary constants. Substituting Eqs. 3.1 and 3.2 and the forced motion in Eq. 2.1 gives

$$
\left(j \omega-\vec{w}_{b} x\right) \vec{v}_{g}=q \vec{E} / m=\vec{a}
$$

whose solution is

$$
\vec{v}_{g}=\frac{\left(j \omega+\vec{\omega}_{b} \times\right) \vec{a}_{\perp}}{\omega_{b}^{2}-\omega^{2}}-\frac{j \vec{a}_{\mid 1}}{\omega}
$$

This represents a three-dimensional elliptical motion, which is, of course, superposed on the helical random motion. At frequencies above the cyclotron frequency the component in a plane perpendicular to $\vec{B}$ has its major axis along $\vec{E}$, and is out of phase with $\vec{E}$. At lower frequencies the reverse is true.

At the cyclotron frequency Eq. 3.4 is singular and there is no steady state. The solution of the equation of motion is

$$
\vec{v}_{g}=\left[\left(\omega t-\frac{j}{2}\right) \vec{E}_{\perp}+\left(j t-\frac{1}{2 \omega}\right) \vec{E} \times \vec{\omega}-j \vec{E}_{\|}\right] \frac{e^{j \omega t}}{B}
$$

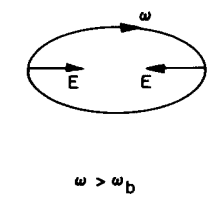

(a)

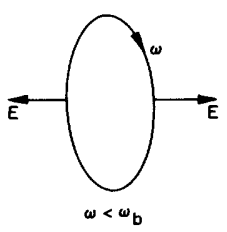

(b)

The vector solution 3.4 is somewhat easier to visualize as a tensor written with the $\mathrm{z}$-axis along $\overrightarrow{\mathrm{B}}$

Fig. 2. Elliptical motion in an alternating electric field. $\vec{B}$ is normal to the paper. (a) Displacement out of phase. (b) Displacement in phase. 


$$
\left\|\mathscr{K}_{\mathrm{k} \ell}\right\|=\left\|\begin{array}{ccc}
\frac{\omega^{2}}{\omega^{2}-\omega_{\mathrm{b}}^{2}} & \frac{j \omega \omega_{\mathrm{b}}}{\omega^{2}-\omega_{\mathrm{b}}^{2}} & 0 \\
\frac{-j \omega_{\mathrm{b}}}{\omega^{2}-\omega_{\mathrm{b}}^{2}} & \frac{\omega^{2}}{\omega^{2}-\omega_{\mathrm{b}}^{2}} & 0 \\
0 & 0 & 1
\end{array}\right\|
$$

Then

$$
\mathrm{v}_{\mathrm{g}, \mathrm{k}}=\frac{-\mathrm{jq}}{\mathrm{m} \omega} \mathscr{K}_{\mathrm{k} \ell} \mathrm{E}_{\ell}
$$

where the usual summation convention over the repeated index is implied.

The velocities of the charged particles determine the permittivity of a plasma; hence its ability to propagate electromagnetic waves, in the following way. From the velocity of the guiding center one obtains the real current at the frequency $\omega$

$$
\mathrm{J}_{\mathrm{k}}=\mathrm{nq} \mathrm{v}_{\mathrm{g}, \mathrm{k}}=\frac{\mathrm{nq}}{\mathrm{m}} \frac{-\mathrm{j}}{\omega} \mathscr{K}_{\mathrm{k} \ell} \mathrm{E}_{\ell}
$$

and the total current is

$$
J+\dot{D}=J+j \omega \epsilon_{o} E
$$

which leads to the permittivity tensor

$$
\epsilon_{\mathrm{k} \ell}=\epsilon_{\mathrm{o}}\left(\delta_{\mathrm{k} \ell}-\frac{\omega_{\mathrm{p}}^{2}}{\omega^{2}} \mathscr{K}_{\mathrm{k} \ell}\right)
$$

where the symbol $\omega_{\mathrm{p}}^{2}=\mathrm{nq}^{2} / \epsilon_{\mathrm{o}} \mathrm{m}$ defines the plasma frequency

$$
\nu_{\mathrm{p}}=\frac{\omega \mathrm{p}}{2 \pi}=8.984 \sqrt{\mathrm{n}} \text { in mks units for electrons }
$$

The ratio of plasma to cyclotron frequency has the interesting property

$$
\frac{\omega_{\mathrm{p}}^{2}}{\omega_{\mathrm{b}}^{2}}=\frac{\mathrm{nm} \mathrm{c}^{2}}{\mathrm{~B} \cdot \mathrm{H}}=\frac{\text { material energy density }}{2 \times \text { magnetic energy density }}
$$


In general, we are not interested in the off-diagonal terms of the permittivity. For electric fields parallel to $\vec{B}$, the dielectric coefficient $\epsilon / \epsilon_{0}$ is less than 1 and is negative for frequencies below the plasma frequency. Electromagnetic waves of these frequencies, therefore, cannot propagate and the plasma frequency represents a lower frequency limit for propagation. For waves whose electric field is perpendicular to the magnetic field $\vec{B}$, there is a forbidden band (1)

$$
\omega_{\mathrm{b}}^{2}<\omega^{2}<\omega_{\mathrm{b}}^{2}+\omega_{\mathrm{p}}^{2}
$$

Waves of frequency above this band propagate at a phase velocity faster than in free space while waves of frequency below the band propagate slower than light.

4. Orbits in a changing magnetic field. In a magnetic field changing at the rate $\vec{B}$ there is an induced electric field $\overrightarrow{\mathrm{E}}_{i}$ and applying the induction law to the circular orbit of the charged particle we have

$$
2 \pi r_{b} E_{i}=\oint \vec{E}_{i} \cdot d \vec{s}=-\dot{\Phi}=-\pi r_{b}^{2} \dot{B}
$$

Consequently, the motion perpendicular to the magnetic field will be accelerated and we have

$$
\dot{\mathrm{v}}_{\perp}=\mathrm{q} \mathrm{E}_{\mathrm{i}} / \mathrm{m}=\mathrm{r}_{\mathrm{b}} \dot{\omega}_{\mathrm{b}} / 2
$$

From Eq. 1.2

$$
\dot{\mathrm{v}}_{\perp}=\mathrm{r}_{\mathrm{b}} \dot{\omega}_{\mathrm{b}}+\dot{\mathrm{r}}_{\mathrm{b}} \omega_{\mathrm{b}}
$$

whence

$$
\omega_{b} \dot{r}_{b}=-r_{b} \dot{\omega}_{b} / 2=-\dot{v}_{\perp}
$$

and

$$
\frac{d}{d t}\left(\omega_{b} r_{b}^{2}\right)=\frac{d}{d t}\left(r_{b} v_{\perp}\right)=r_{b}\left(\omega_{b} \dot{r}_{b}+\dot{v}_{\perp}\right)=0
$$

This equation expresses the conservation of angular momentum of the ion about the guiding center, or, alternatively, the conservation of magnetic flux linked by the circular motion. As the magnetic field $B$ is increased, the orbits decrease in size and also move closer together, each guiding center remaining on a line of force and each orbit on a tube of force. The density of charged particles, as well as their energy, therefore increases proportionately to the magnetic field $B$.

$$
\frac{d}{d t}\left(\frac{u_{\perp}}{B}\right)=\frac{d}{d t}\left(\frac{n}{B}\right)=0
$$


where $u_{\perp}=\frac{1}{2} m v_{\perp}^{2}$ is the kinetic energy of the circular motion.

\section{b. Orbits in Inhomogeneous Magnetic Fields}

We shall now consider certain peculiarities of ionic orbits which appear in inhomogeneous magnetic fields that have been of particular interest to astronomers. They have been treated by H. Alfvén (2). We shall always assume small gradients, grad B $\ll \mathrm{B} / \mathrm{r}_{\mathrm{b}}$, so that the circular motion is not sufficiently deformed to prevent the treatment in terms of the motion of a guiding center.

5. Longitudinal magnetic gradient. Consider first the effect of a gradient $(\vec{B} \cdot \operatorname{grad} B)$ such that the magnitude of $B$ changes along a straight line of force. The particle then observes a uniform magnetic field but one which changes with time as the guiding center moves along a line of force. Accordingly, the conditions of the last section hold in a coordinate system moving with the guiding center and the orbit of the particle will link a constant amount of magnetic flux (Busch's

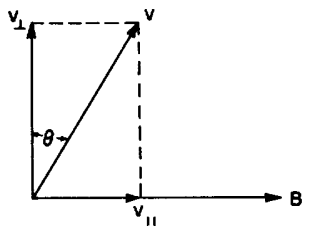
theorem). The orbit is then a spiral described on the surface of a tube of force. The pitch of the spiral, $2 \pi r_{b} v_{\|} / v_{\perp}=2 \pi r_{b}$ $\tan \theta$, changes, however, as the conservation of energy requires $\mathrm{u}=$ constant, and conservation of angular momentum requires $u_{\perp} / B=$ constant. Combining these two gives

Fig. 3. The pitch angle $\theta$.

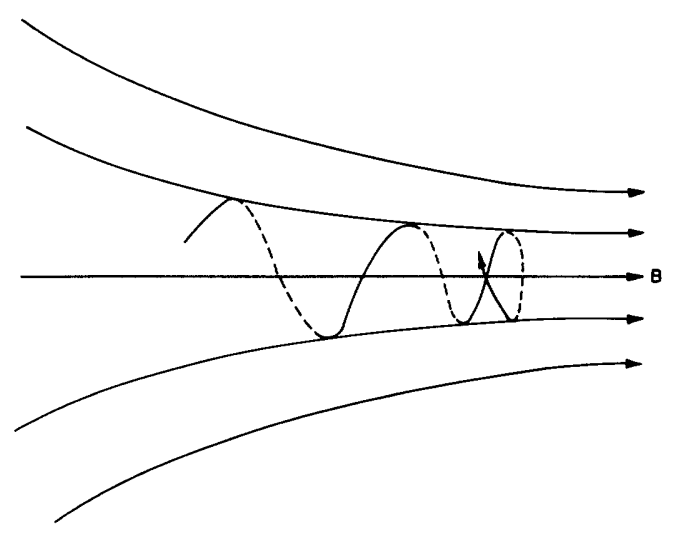

Fig. 4. Reflection of a spiral path in a converging magnetic field.

$$
\frac{B u}{u_{\perp}}=\frac{B v^{2}}{v_{\perp}^{2}}=B \sec ^{2} \theta=B_{0}
$$

where $\mathrm{B}_{\mathrm{O}}$ is a constant. An ion spiraling along a tube of force in the direction of increasing field has a decreasing pitch angle $\theta$. $B_{0}$ is the value of $B$ at which the angle $\theta$ goes through zero and therefore at which the motion of the particle is reflected. Reciprocally, if there is a distribution of particles at B with various pitch angles, only particles for which

$$
\cos ^{2} \theta<\frac{\mathrm{B}}{\mathrm{B}_{\mathrm{O}}}
$$

will penetrate to fields greater than $B_{O}$. 
From (5.1) it readily follows that

$$
v_{\|}^{2}=v^{2} \frac{B_{0}-B}{B_{0}}
$$

whence the equation of motion of the guiding center along the line of force is

$$
v t=\int \sqrt{\frac{B_{0}}{B_{0}-B}} d z
$$

6. Transverse gradients. We must now consider two effects of magnetic gradients which produce motion of the guiding center at right angles to the magnetic field. If there is a magnetic gradient at right angles to the magnetic field, the erstwhile circular orbit will no longer be circular as the curvature will now be greater on one side than on the other and this will produce a crosswise motion, illustrated in Fig. 5. Similarly, if the lines of force are curved, the vector curvature is given by

$$
\left(\frac{1}{\vec{R}}\right)=\frac{(\vec{B} \cdot \text { grad }) \vec{B}_{\perp}}{\vec{B} \cdot \vec{B}}=\frac{1}{B} \frac{\partial \vec{B}_{\perp}}{\partial z}
$$

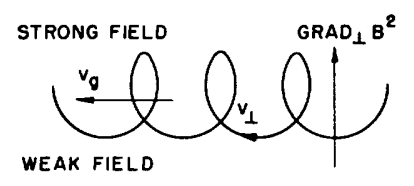

Fig. 5. Cross-drift in a magnetic gradient. $\vec{B}$ is perpendicular to the paper.
(Note that, although $\mathrm{B}_{\perp}$, the component of $\mathrm{B}$ perpendicular to itself, is zero, its gradient is not zero when the lines of $\vec{B}$ are curved.) The centrifugal force caused by the motion of the guiding center along the line will produce an additional motion of the guiding center at right angles to the magnetic field and to the curvature.

We shall assume that the gradients are small so that we can expand the magnetic field about the center of the almost circular orbit

$$
\vec{\omega}_{b}=\vec{\omega}_{o}+\left(\vec{r}_{b} \cdot \operatorname{grad}\right) \vec{\omega}_{o}
$$

and we shall assume

$$
\vec{v}=\vec{v}_{g}+\vec{\omega}_{o} \times \vec{r}_{b}
$$

as before. Substituting in the equation of motion gives 


$$
\begin{aligned}
\vec{\omega}_{\mathrm{b}} \times \overrightarrow{\mathrm{v}}= & \vec{\omega}_{\mathrm{O}} \times \overrightarrow{\mathrm{v}}_{\mathrm{g}}+\vec{\omega}_{\mathrm{O}} \times\left(\vec{\omega}_{\mathrm{o}} \times \overrightarrow{\mathrm{r}}_{\mathrm{b}}\right)+\left(\overrightarrow{\mathrm{r}}_{\mathrm{b}} \cdot \operatorname{grad}\right)\left(\vec{\omega}_{\mathrm{o}} \times \overrightarrow{\mathrm{v}}_{\mathrm{g}}\right) \\
& -\left(\vec{\omega}_{\mathrm{O}} \times \overrightarrow{\mathrm{r}}_{\mathrm{b}}\right) \times\left(\overrightarrow{\mathrm{r}}_{\mathrm{b}} \cdot \operatorname{grad}\right) \vec{\omega}_{\mathrm{O}} \\
= & \dot{\overrightarrow{\mathrm{v}}}=-\omega_{\mathrm{O}}^{2} \overrightarrow{\mathrm{r}}_{\mathrm{b}}+\left(\frac{\mathrm{v}_{\mathrm{II}}}{\mathrm{B}}\right)^{2}(\overrightarrow{\mathrm{B}} \cdot \operatorname{grad}) \overrightarrow{\mathrm{B}}_{\perp}
\end{aligned}
$$

Expanding the triple cross products and remembering that $\overrightarrow{\mathrm{r}}_{\mathrm{b}} \cdot \vec{\omega}_{\mathrm{O}}=0$, we find

$$
\vec{\omega}_{\mathrm{o}} \times \vec{v}_{\mathrm{g}}+\left(\overrightarrow{\mathrm{r}}_{\mathrm{b}} \cdot \operatorname{grad}\right)\left(\vec{\omega}_{\mathrm{o}} \times \vec{v}_{\mathrm{g}}\right)-\frac{1}{2} \overrightarrow{\mathrm{r}}_{\mathrm{b}}\left(\mathrm{r}_{\mathrm{b}} \cdot \operatorname{grad} \omega_{\mathrm{o}}^{2}\right)=\left(\frac{\mathrm{v}_{\| 1}}{\mathrm{~B}}\right)^{2}(\overrightarrow{\mathrm{B}} \cdot \operatorname{grad}) \overrightarrow{\mathrm{B}}_{\perp}
$$

This equation contains terms of frequency $\omega_{0}$ because that part of the motion which has been assumed circular in Eq. 6.3 is not truly circular. These periodic terms are eliminated by averaging over a cycle which gives

$$
\overline{\vec{r}}_{b}=0
$$

and

$$
\overline{\vec{r}_{\mathrm{b}}\left(\overrightarrow{\mathrm{r}}_{\mathrm{b}} \cdot \operatorname{grad} \omega_{\mathrm{o}}^{2}\right)}=\left(\frac{\mathrm{r}_{\mathrm{b}}^{2}}{2}\right) \operatorname{grad}_{\perp} \omega_{\mathrm{o}}^{2}
$$

When these are substituted in Eq. 6.5, one obtains

$$
\left.\begin{array}{rl}
\vec{\omega}_{\mathrm{O}} \times \overrightarrow{\mathrm{v}}_{\mathrm{g}} & =\left(\frac{\mathrm{r}_{\mathrm{b}}^{2}}{4}\right)\left(\operatorname{grad}_{\perp} \omega_{\mathrm{O}}^{2}\right)+\left(\frac{\mathrm{v}_{\|}}{\mathrm{B}}\right)^{2}(\overrightarrow{\mathrm{B}} \cdot \operatorname{grad}) \overrightarrow{\mathrm{B}}_{\perp} \\
& =\left(\frac{\mathrm{v}_{\perp}}{2 \mathrm{~B}}\right)^{2}\left(\operatorname{grad}_{\perp} \mathrm{B}^{2}\right)+\left(\frac{\mathrm{v}_{\Perp l}}{\mathrm{~B}}\right)^{2}(\overrightarrow{\mathrm{B}} \cdot \operatorname{grad}) \overrightarrow{\mathrm{B}}_{\perp}
\end{array}\right\}
$$

The two terms on the right represent a magnetic pressure and a centrifugal force. As the force is independent of the sign of $q$, the velocity of the guiding center $\mathrm{v}_{\mathrm{g}}$ does depend on the sign of $\mathrm{q}$; that is, in a neutral medium these forces will produce a current or a polarization of the medium.

If we introduce the current density $\vec{J}$ in the medium by the relation

$$
\vec{J} \times \vec{B}=(\vec{B} \cdot \operatorname{grad}) \vec{H}_{\perp}-\frac{1}{2} \operatorname{grad}_{\perp} \vec{B} \cdot \vec{H}
$$


Equation 6.8 can be transformed into

$$
\vec{\omega}_{\mathrm{O}} \times \vec{v}_{\mathrm{g}}=\frac{v_{\perp}^{2}+2 \text { vोl }}{4 \mathrm{~B}^{2}} \operatorname{grad}_{\perp} \mathrm{B}^{2}+\frac{v^{2}}{\overrightarrow{\mathrm{B}} \cdot \overrightarrow{\mathrm{H}}} \overrightarrow{\mathrm{J}} \times \overrightarrow{\mathrm{B}}
$$

which relates the force on a particle $m \vec{\omega}_{\mathrm{o}} \times \vec{v}_{\mathrm{g}}$ to the force per unit volume $\overrightarrow{\mathrm{J}} \times \overrightarrow{\mathrm{B}}$ and in addition to the normal gradient of $\mathrm{B}^{2}$. Solving for the velocity of the guiding center gives

$$
\vec{v}_{g}=\frac{u_{\perp}+2 u_{\|}}{2 q} \frac{\vec{B} \times \operatorname{grad~B}}{B^{4}}+\frac{2 u_{\|}}{q} \frac{\vec{B} \times(\vec{J} \times \vec{B})}{B^{2} \vec{H} \cdot \vec{B}}+\vec{v}_{\|}
$$

This equation simplifies considerably if there is no current $(J=0)$ and if it is applied to an isotropic distribution in such a way that

$$
u_{\perp}=2 u_{\|}=k T
$$

where the factor 2 comes from the two directions perpendicular to $\overrightarrow{\mathrm{B}}$. The expression then reduces to

$$
\vec{v}_{g}=\frac{k T}{q} \frac{\vec{B} \times \operatorname{grad} B^{2}}{B^{4}}+\vec{v}_{\|}
$$

These relations have been given by Spitzer (3).

The velocity of the guiding center at right angles to the magnetic field $\vec{B}$ is in such a direction that the magnetic field of the current carried by $\vec{v}_{g}$ reduces the inhomogeneity in $\vec{B}$. If the charges carried by $\vec{v}_{g}$ are allowed to accumulate, they will produce an electric field which in turn will produce mass motion in the direction of lower magnetic fields.

7. The drift velocity. The drift velocity $\vec{v}_{d}$ is defined as the average velocity of the particles contained in a small volume V. More generally, it is the limit of this as the volume $\mathrm{V}$ becomes small, but not smaller than the radius $r_{b}$. The drift velocity $\vec{v}_{d}$ is to be distinguished very carefully from the velocity $\vec{v}_{g}$, which is the time average of the velocity of a single particle. These two velocity averages differ because the walls of the volume $\mathrm{V}$ cut through some orbits so that $\mathrm{V}$ contains only parts of the orbits of certain particles and when there are magnetic gradients these fractional orbits correspond to velocities greater than $\vec{v}_{g}$ on one side of $V$ and smaller than, or even opposed to, $\vec{v}_{g}$ on the other side. For a uniform magnetic field these fractional orbits on the two sides may 


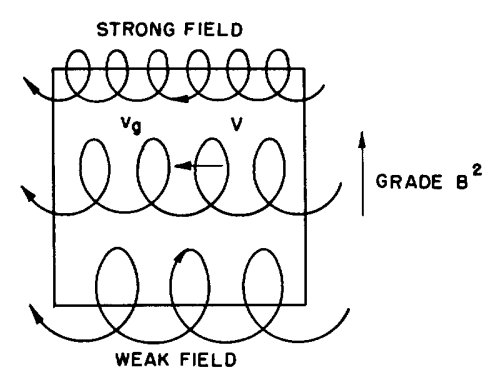

Fig. 6. A volume $\mathrm{V}$ contains fractional orbits for which the motion is opposed to the guiding center. be added to give complete orbits so that $\vec{v}_{d}=\vec{v}_{g}$, but in this case $\vec{v}_{g}=0$ and both aver ages vanish in directions normal to B. For an inhomogeneous field there are more fractional orbits on the weak side, assuming uniform particle density, because these orbits are larger. But these fractional orbits correspond to motion against $\vec{v}_{\mathrm{g}}$, and the refore $\overrightarrow{\mathrm{v}}_{\mathrm{d}}<\overrightarrow{\mathrm{v}}_{\mathrm{g}}$. A careful computation shows (3) that $\vec{v}_{d}=0$ and we shall not repeat the argument, but merely point out that this result must be so, by considering a system in

thermodynamic equilibrium. In such a system even when there are magnetic gradients present, provided there are no other forces, the density is uniform. Any drift velocities would disturb this uniformity and therefore must be zero. This cancellation of $\vec{v}_{g}$ by the intercepted orbits is exactly the same as the cancellation of the diamagnetism of free electrons arising from surface effects (4).

In a physical enclosure with real walls, the situation may be different and it depends on the nature of the walls. If they are perfectly reflecting, so that equilibrium is possible, $\vec{v}_{d}=0$ even though $\vec{v}_{g}$ is different from zero as above. The particles intercepted by the wall hop along the wall in the reverse direction to $\vec{v}_{g}$ and this produces the cancellation. If charged particles striking the walls are absorbed by it, this eliminates the surface currents discussed above and then $\vec{v}_{d} \approx \vec{v}_{g}$, but the distribution of these velocities is different: the drift velocity is confined to the region near the walls until density gradients are generated, and then the problem becomes more complicated.

In the case of electric fields studied in Sec. 2, $\vec{v}_{d}$ and $\vec{v}_{g}$ are equal. Henceforth we shall deal with assembly averages, and therefore with $\vec{v}_{d}$ and not with $\vec{v}_{g}$.

\section{c. Pressure Gradients}

8. Equation for the drift velocity. Pressure gradients are exactly the opposite of magnetic gradients, as they produce no motion of the individual particles $\left(\vec{v}_{\mathrm{g}}=0\right)$, unless the particles collide with each other, but they do produce a drift velocity $\vec{v}_{d}$. Thus we have the following scheme: 


$$
\begin{aligned}
& \text { Type of Force Velocity Average } \\
& \overrightarrow{\mathrm{E}} \quad \overrightarrow{\mathrm{v}}_{\mathrm{g}}=\overrightarrow{\mathrm{v}}_{\mathrm{d}} \\
& \operatorname{grad} \mathrm{B}^{2} \quad \overrightarrow{\mathrm{v}}_{\mathrm{g}} \neq 0, \overrightarrow{\mathrm{v}}_{\mathrm{d}}=0 \\
& \operatorname{grad} \mathrm{p} \quad \overrightarrow{\mathrm{v}}_{\mathrm{g}}=0, \quad \overrightarrow{\mathrm{v}}_{\mathrm{d}} \neq 0
\end{aligned}
$$

In dealing with an assembly whose velocity distribution is isotropic, a pressure gradient is equivalent to a force, so that one can write the equation for the drift velocity

$$
\mathrm{m} \dot{\vec{v}}_{d}=q\left(\vec{E}+\vec{v}_{d} \times \vec{B}\right)-\frac{\operatorname{grad} p}{n}
$$

where

$$
\mathrm{p}=\frac{1}{3} \mathrm{~nm} \overline{\mathrm{v}^{2}}=\mathrm{nkT}
$$

Thus $\vec{v}_{d}$ is obtained from a force equation exactly like that for a single particle. $\vec{v}_{d}$ determines an orbit which shall be called the orbit of an average ion, to distinguish it from the average orbit of a given ion which is determined by $\vec{v}_{\mathrm{g}}$. Equation 8.1 can be solved for $\overrightarrow{\mathrm{v}}_{\mathrm{d}}$ and it gives

$$
\vec{v}_{d}=\left(\vec{E}-\frac{\operatorname{grad} p}{n q}\right) \times \frac{\vec{B}}{B^{2}}+\vec{v}_{\|}
$$

and

$$
\mathrm{m} \dot{\vec{v}}_{\|}=\mathrm{q} \overrightarrow{\mathrm{E}}_{\|}-\frac{1}{\mathrm{n}} \frac{\partial \mathrm{p}}{\partial \mathrm{z}}
$$

We have neglected here a term in $\overrightarrow{\mathrm{B}} \times \dot{\vec{v}}_{\mathrm{d}}$. This term would give the circular motion of Sec. 1 and would be applicable if all the ions of the assembly had the same starting conditions. If the starting conditions are random, the phases of the circular motions will be random, and the $\vec{B} \times \dot{\vec{v}}_{d}$ term averages out.

9. Equilibrium. In the case of thermodynamic equilibrium, $\overrightarrow{\mathrm{v}}_{\mathrm{d}}=0$ and Eqs. 8.3 and 8.4 lead to

$$
\mathrm{qE}=\frac{\operatorname{grad} \mathrm{p}}{\mathrm{n}}=\mathrm{k} \mathrm{T} \frac{\operatorname{grad} \mathrm{n}}{\mathrm{n}}
$$

or

$$
\mathrm{n}=\mathrm{n}_{\mathrm{o}} \exp (-\mathrm{qV} / \mathrm{k} \mathrm{T})
$$


Equations 8.3 and 8.4 are therefore consistent with a Boltzmann distribution that is entirely independent of the magnetic field.

10. Stationary distributions. We shall now assume a stationary state in which $\overrightarrow{\mathrm{v}}_{\mathrm{d}}$ is constant, and

$$
\vec{J}=n q \vec{v}_{d}=\operatorname{curl} \vec{H}
$$

that is, there are no currents other than those arising from the particles in question. This is somewhat different from the assumption of Sec. 6 where the magnetic field was given and assumed to arise from external sources. Equation 8.1 then gives

$$
\operatorname{grad} p=n q \vec{E}-\vec{B} \times \operatorname{curl} \vec{H}
$$

or

$$
\operatorname{grad}\left(p+\frac{1}{2} \vec{B} \cdot \vec{H}\right)=n q \vec{E}+(\vec{B} \cdot \operatorname{grad}) \vec{H}
$$

Two special cases with $\mathrm{E}=0$ will be considered.

(a) Straight parallel magnetic field lines: In this case, the right-hand side of Eq. 10.3 vanishes and we have (5)

$$
\mathrm{p}+\frac{1}{2} \overrightarrow{\mathrm{B}} \cdot \overrightarrow{\mathrm{H}}=\text { constant }
$$

The charged particles responsible for the current concentrate in the regions of weak field, the total material plus magnetic pressure being a constant. The process by which this concentration is reached is that described in Sec. 6. Perhaps the important thing about the rather logical result 10.4 is that it is not correct unless $(\vec{B} \cdot \operatorname{grad}) \overrightarrow{\mathrm{H}}$ vanishes. This is illustrated by the following example.

$(\beta)$ Circular magnetic field lines: W. H. Bennett (6) first studied the dynamic equilibrium of an electrically neutral double stream of cylindrical symmetry. We assume equal numbers of positive and negative charges, $n_{+}=n_{-}=n$, with the relative velocity $\vec{v}_{d}$, so that the current density is $J=n \mathrm{qv}_{\mathrm{d}}$. Bennett finds that the equilibrium current distribution is

$$
J=\frac{J_{0}}{\left(1+b r^{2}\right)^{2}}
$$

where $J_{0}$ is the current density on the axis, and $b$ is a parameter determining the size of the stream. The field of this stream is 


$$
\mathrm{H}=\frac{\mathrm{J}_{\mathrm{o}} \mathrm{r} / 2}{1+\mathrm{b} \mathrm{r}^{2}}
$$

and the force equation 10.2 is readily integrated to give

$$
\mathrm{p}=\frac{\mu_{\mathrm{o}} \mathrm{J}_{\mathrm{O}}^{2} / 8 \mathrm{~b}}{(1+\mathrm{br})^{2}}=\frac{\mu_{\mathrm{O}} \mathrm{J}_{\mathrm{o}}}{8 \mathrm{~b}} \mathrm{~J}
$$

with no constant of integration if the pressure is to vanish at infinity. It is noted that $p+\frac{1}{2} \vec{B} \cdot \vec{H}$ is not constant. The proportionality between $p=2 n k T$ and $J$ allows the relative drift velocity

$$
\mathrm{v}_{\mathrm{d}}=16 \mathrm{bk \textrm {T } / \mu _ { \mathrm { o } }} \mathrm{q} \mathrm{J}_{\mathrm{o}}
$$

to be constant over the cross section of the stream, which is essential if particles are to move freely.

The total current in the stream is found by integrating Eq. 10.5

$$
\mathrm{I}=\frac{\pi \mathrm{J}}{\mathrm{b}}
$$

and by integrating the pressure Eq. 10.7, one finds

$$
\int_{0}^{\infty} \mathrm{p} 2 \pi \mathrm{rdr}=\mathrm{Nk \textrm {T }}=\frac{\mu_{\mathrm{O}} \mathrm{I}^{2}}{8 \pi}
$$

where $\mathrm{N}$ is the total number of particles, positive and negative, per unit length of stream. Given $\mathrm{N}$ and T, Eq. 10.10 fixes a critical current $\mathrm{I}$ for equilibrium. If the current is less than this, the magnetic force is insufficient to contain the thermal pressure and the stream expands. Conversely, a larger current is focused. 


\section{THE LANGEVIN EQUATION}

\section{a. Mobility}

11. Relaxation times. Langevin (7) added to the steady forces acting on an ion a stochastic force $\mathrm{m} \overrightarrow{\mathrm{A}}(\mathrm{t})$, caused by collisions with the molecules of a gas, so that

$$
\left.\begin{array}{l}
\dot{\vec{v}}=\vec{a}+\vec{\omega}_{b} \times \vec{v}+\vec{A}(t), \\
\vec{a}=q \vec{E} / m, \quad \vec{\omega}_{b}=-q \vec{B} / m
\end{array}\right\}
$$

where $\vec{A}(t)$ cannot be specified because it is fluctuating randomly. However, certain average properties of $\vec{A}(t)$ may be known and we shall assume that these are

$$
\begin{aligned}
\overrightarrow{\vec{A}} & =-\nu_{\mathrm{c}} \overrightarrow{\mathrm{v}}_{\mathrm{g}}, \\
\mathrm{m} \overrightarrow{\mathrm{v}} \cdot \overrightarrow{\mathrm{A}} & =\lambda \nu_{\mathrm{c}}(\overline{\mathrm{U}}-\mathrm{u})
\end{aligned}
$$

In Eq. $27.6 \nu_{\mathrm{c}}$ will be shown to be the collision frequency for momentum transfer and $\lambda$ (Eqs. 25.2 and 41.9) an energy loss parameter which, for elastic collisions, is

$$
\lambda=\frac{2 \mathrm{~m}}{(\mathrm{M}+\mathrm{m})}
$$

where $m$ is the mass of the ion and $M$ that of a molecule of the gas. Therefore, $\lambda$ is very small for an electron but it is of the order 1 for an ion; $u$ is the energy of the ion; and $\bar{U}$ is the average energy of the gas molecules. The bar over $\vec{A}$ and $\overrightarrow{\vec{v}} \cdot \vec{A}$ represents a time average during the motion of a single particle, over a time long compared with $1 / \nu_{c}$ but during which $\nu_{c}$, and therefore $u$, does not change appreciably (8). 'This is an impossible condition for ions, but it may be satisfied for electrons.

The results of this section, using the Langevin equation, will be functions of $u$, the instantaneous energy of the electron. They will not be directly applicable to an assembly having a distribution of energy, but relate to a "microcanonical" assembly with a known energy. These results will fit into the Boltzmann theory before integration over energies.

Omitting for the moment all external forces, the solution of Eqs. 11.1 is found by first multiplying both sides by $\mathrm{m} \overrightarrow{\mathrm{v}}$ and averaging. This yields, making use of 11.3 , 


$$
\frac{d u}{u-\bar{U}}=-\lambda \nu_{c} d t
$$

$1 /\left(\lambda \nu_{c}\right)$ is the relaxation time for energy and, when the energy dependence of $\nu_{c}(u)$ is known, Eq. 11.5 can be integrated for $u(t)$.

Averaging Eq. 11.1 directly yields

$$
\frac{d v_{g}}{v_{g}}=-\nu_{c} d t
$$

so that $1 / \nu_{c}$ is the relaxation time for momentum. Since $\nu_{c}(t)$ is known, it can be integrated for $v_{g}(t)$.

Since we shall not consider magnetic gradients, the subscripts g or $d$ are rather arbitrary, so that the energy associated with $v_{g}$ will be denoted $u_{d}=\frac{1}{2} \operatorname{mv}_{g}^{2}$ for convenience later. The total energy $u$ is then conveniently divided into "drift" and "random" energy

$$
\mathrm{u}=\mathrm{u}_{\mathrm{d}}+\mathrm{u}_{\mathrm{r}}
$$

The drift energy disappears, on the average, at each collision, most of it being converted into random energy and a small fraction $\lambda$ going to the gas.

12. Mobility. Reintroducing the electric and magnetic fields and averaging Eq. 11.1 over the time gives

$$
\dot{\vec{v}}_{\mathrm{g}}=\overrightarrow{\mathrm{a}}+\vec{\omega}_{\mathrm{b}} \times \overrightarrow{\mathrm{v}}_{\mathrm{g}}-\nu_{\mathrm{c}} \overrightarrow{\mathrm{v}}_{\mathrm{g}}
$$

We shall consider the case of an alternating electric field $\vec{E} \sim e^{j \omega t}$ as the direct current case is readily derived from the ac solution. The velocity $\vec{v}_{g}$ then also varies as $\mathrm{e}^{\mathrm{j \omega t}}$ and Eq. 12.1 becomes

$$
\left(\nu_{c}+j \omega-\vec{\omega}_{b} x\right) \vec{v}_{g}=\vec{a}
$$

whence

$$
\left(\nu_{c}+j \omega\right) v_{g}^{2}=\vec{a} \cdot \vec{v}_{g}
$$

The component of the field $\vec{E}$ in the direction of the drift is independent of the magnetic field, even though the drift may be partially across the magnetic field. This is Tonks' theorem (9). Solving Eq. 12.2 for the velocity $\mathrm{v}_{\mathrm{g}}$ gives 


$$
\left[\nu_{c}+j\left(\omega-\omega_{b}\right)\right]\left[\nu_{c}+j\left(\omega+\omega_{b}\right)\right] \vec{v}_{g}-\left(\vec{\omega}_{b} \cdot \vec{v}_{g}\right) \vec{\omega}_{b}=\left(\nu_{c}+j \omega+\vec{\omega}_{b} \times\right) \vec{a}
$$

This vector equation is somewhat better written in tensor notation

$$
\mathrm{v}_{\mathrm{g}, \mathrm{i}}=\mu_{\mathrm{ik}} \mathrm{E}_{\mathrm{k}}
$$

If we introduce the symbols*

$$
\ell=\frac{1}{\nu_{c}+j\left(\omega+\omega_{b}\right)}, \quad r=\frac{1}{\nu_{c}+j\left(\omega-\omega_{b}\right)}, \quad p=\frac{1}{\nu_{c}+j \omega}
$$

to correspond to left and right circular polarizations of the field normal to $\vec{B}$, and $\vec{E}$ parallel to $\vec{B}$, the mobility tensor $\mu_{i k}$ is

$$
\mu_{i k}=\frac{q}{2 m}\left\|\begin{array}{ccc}
\ell+r & -j(\ell-r) & 0 \\
(\ell-r) & \ell+r & 0 \\
0 & 0 & 2 p
\end{array}\right\|
$$

It is convenient to refer to the three tensor components as the

and

$$
\begin{array}{ll}
\text { parallel } & \mu_{\|}=\frac{\mathrm{pq}}{\mathrm{m},} \\
\text { transverse } & \mu_{T}=\frac{\boldsymbol{\ell}+\mathrm{r}}{2} \frac{\mathrm{q}}{\mathrm{m}}
\end{array}
$$

$$
\text { perpendicular } \quad \mu_{\perp}=j \frac{\ell-r}{2} \frac{q}{m}
$$

components.

The energy of the drift motion when it is at right angles to the magnetic field is obtained by replacing $\mathrm{e}^{j \omega t}$ by $\cos \omega t$ and then squaring Eq. 12.5. It contains, of course, terms of double frequency.

$$
u_{d}(t)=\frac{m a^{2}}{4} \frac{\left(\nu_{c}^{2}+\omega^{2}+\omega_{b}^{2}\right)+\left(\nu_{c}^{2}-\omega^{2}+\omega_{b}^{2}\right) \cos 2 \omega t+2 \nu_{c} \omega \sin 2 \omega t}{\left[\nu_{c}^{2}+\left(\omega-\omega_{b}\right)^{2}\right]\left[\nu_{c}^{2}+\left(\omega+\omega_{b}\right)^{2}\right]}
$$

*Note that $\omega_{b}$ is negative for an ion but positive for an electron. Resonance occurs for a field rotating in the right-hand direction around the magnetic field when electrons are responsible. 
Averaging over the time eliminates the periodic terms and gives for the average kinetic energy of the drift motion the simpler expression

$$
u_{d}=\frac{m a^{2}}{8}\left[\frac{1}{\nu_{c}^{2}+\left(\omega-\omega_{b}\right)^{2}}+\frac{1}{\nu_{c}^{2}+\left(\omega+\omega_{b}\right)^{2}}\right]
$$

Simpler expressions are obtained in the special case of a constant field $(\omega=0)$ when the mobility tensor is

$$
\mu_{i k}=\frac{q}{m}\left\|\begin{array}{ccc}
\frac{\nu_{c}}{\nu_{c}^{2}+\omega_{b}^{2}} & \frac{\omega_{b}}{\nu_{c}^{2}+\omega_{b}^{2}} & 0 \\
\frac{-\omega_{b}}{\nu_{c}^{2}+\omega_{b}^{2}} & \frac{\nu_{c}}{\nu_{c}^{2}+\omega_{b}^{2}} & 0 \\
0 & 0 & \frac{1}{\nu_{c}}
\end{array}\right\|
$$

and the drift energy is

$$
u_{d}=\frac{m a^{2}}{2\left(\nu_{c}^{2}+\omega_{b}^{2}\right)}
$$

Equation 12.11 seems to differ from Eq. 12.9 by a factor 2, but this comes about because the acceleration a in Eq. 12.9 is defined in terms of the peak instead of the root-meansquare field.

Similarly, in the absence of a magnetic field, the mobility becomes a scalar

$$
\mu=\frac{q / m}{\nu_{c}+j \omega}
$$

and the drift energy is

$$
u_{d}=\frac{q^{2} E_{p}^{2} / 4 m}{\nu_{c}^{2}+\omega^{2}}=\frac{q^{2} E_{e f f}^{2}}{2 m \nu_{c}^{2}}
$$

This last equation serves as the definition of an "effective" field $\mathbf{E}_{\text {eff }}$ which incorporates both the factor $\frac{1}{2}$ of an average cosine squared and the factor $\nu_{c}^{2} /\left(\nu_{c}^{2}+\omega^{2}\right)$ by which the drift energy in an alternating field is reduced from that in a constant field. 


\section{b. Diffusion}

13. The random flight. Diffusion currents are not obtained directly from the Langevin equation because this equation treats the average motion of a single particle, and it has been shown that $\vec{v}_{g}=0$ under a concentration gradient. A diffusion coefficient may, however, be defined in terms of the mean square displacement of a single particle through the theory of the Random Flight (10). The procedure is as follows:

The motion of a free ion or electron in the absence of an electric field is along a helix, so that the displacement $\vec{r}$ is the chord of a helix. That is, $\vec{r}_{\perp}$ is the chord of a circle and $z=v_{\|}{ }^{t}$. In moving along the helix, collisions occur with the frequency $\nu_{c}$ so that the probability of having escaped a collision after a time $t$ is $e^{-\nu_{c} t}$. One can then define a vector mean free path

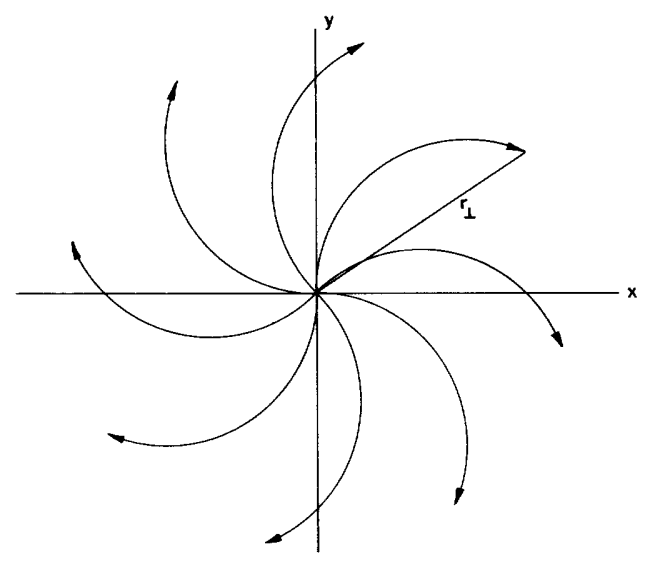

Fig. 7. Orbits considered in defining the mean-square free path.

$$
\ell_{\mathrm{c}}=\frac{\mathrm{v}}{\nu_{\mathrm{c}}}
$$

$$
\vec{l}_{c}=\int_{0}^{\infty} \overrightarrow{\mathrm{r}} \mathrm{e}^{-\nu_{c} \mathrm{t}} \nu_{c} \mathrm{dt}
$$

and a particular mean-square free path

$$
\ell_{i k}^{2}=\int_{0}^{\infty} r_{i} r_{k} e^{-\nu_{c} t} \nu_{c} d t
$$

The scalar mean free path $\ell_{c}$ will be defined in terms of particles moving along the magnetic field,

and we also define a tensor mean-square free path $\overline{\ell_{i k}^{2}}$ by averaging Eq. 13.2 over initial orientations that are assumed to be isotropic:

$$
\overline{l_{\mathrm{zz}}^{2}}=2 \frac{\overline{\mathrm{v}_{\|}^{2}}}{\nu_{\mathrm{c}}^{2}}=\frac{2}{3} \frac{\mathrm{v}^{2}}{\nu_{\mathrm{c}}^{2}}=\frac{2}{3} \ell_{\mathrm{c}}^{2}
$$




$$
\begin{aligned}
& \overline{\ell_{\mathrm{x} x}^{2}}+\overline{\ell_{\mathrm{y} y}^{2}}=\frac{\overline{2 \mathrm{v}_{\perp}^{2}}}{\nu_{\mathrm{c}}^{2}+\omega_{\mathrm{b}}^{2}}=\frac{4}{3} \frac{\mathrm{v}^{2}}{\nu_{\mathrm{c}}^{2}+\omega_{\mathrm{b}}^{2}}=\frac{4}{3} \frac{\nu_{\mathrm{c}}^{2}}{\nu_{\mathrm{c}}^{2}+\omega_{\mathrm{b}}^{2}} \ell_{\mathrm{c}}^{2} \\
& \overline{l_{\mathrm{x} y}^{2}}=\overline{l_{\mathrm{yx}}^{2}}=\overline{l_{\mathrm{zx}}^{2}}=0
\end{aligned}
$$

The tensor is, by its definition, symmetric and the components at right angles to the magnetic field are reduced in the same ratio as the effective field, $\nu_{c}^{2} /\left(\nu_{c}^{2}+\omega_{b}^{2}\right)$, because of the curvature of the orbit. In the limit of high magnetic fields (or low pressures) $\overline{\ell_{\mathrm{x} x}^{2}}+\overline{\ell_{\mathrm{y} y}^{2}}=2 \mathrm{r}_{\mathrm{b}}^{2}$. The mean-square free path at right angles to the field is at most twice the orbit radius squared.

The mean-square displacement tensor at the end of $\mathrm{N}_{\mathrm{C}}=\nu_{\mathrm{c}} \mathrm{t}$ collisions is

$$
\overline{r_{i} r_{k}}=\nu_{c} t \overline{l_{i k}^{2}}
$$

and the symmetric diffusion tensor may be defined (11) by

$$
\mathrm{D}_{\mathrm{i} \mathrm{k}}=\frac{\overline{\mathrm{r}_{\mathrm{i}} \mathrm{r}_{\mathrm{k}}}}{2 \mathrm{t}}=\frac{v^{2}}{3} \| \begin{array}{ccc}
\frac{\nu_{\mathrm{c}}}{\nu_{\mathrm{c}}^{2}+\omega_{\mathrm{b}}^{2}} & 0 & 0 \\
0 & \frac{\nu_{\mathrm{c}}}{\nu_{\mathrm{c}}^{2}+\omega_{\mathrm{b}}^{2}} & 0 \\
0 & 0 & \frac{1}{\nu_{\mathrm{c}}}
\end{array} \mid
$$

It is frequently convenient to think of a magnetic field as increasing the effective size of the container in direction perpendicular to the field. Thus a cavity whose normal diffusion length $\Lambda$ is given by

$$
\frac{1}{\Lambda^{2}}=\frac{1}{\Lambda_{x}^{2}}+\frac{1}{\Lambda_{y}^{2}}+\frac{1}{\Lambda_{z}^{2}}
$$

will, in the presence of a magnetic field along the $z$-axis, have an effective diffusion length $\Lambda_{\text {eff }}$ given by

$$
\frac{1}{\Lambda_{b}^{2}}=\frac{\nu_{c}^{2}}{\nu_{c}^{2}+\omega_{b}^{2}}\left(\frac{1}{\Lambda_{x}^{2}}+\frac{1}{\Lambda_{y}^{2}}\right)+\frac{1}{\Lambda_{z}^{2}}
$$


The diffusion equation in such a cavity is then

$$
\frac{\partial n}{\partial t}=\sum_{1}^{3} \frac{\partial^{2}}{\partial x_{i}^{2}}\left(D_{i i} n\right)=-\frac{D n}{\Lambda_{b}^{2}}
$$

where $\mathrm{D}=\mathrm{v}^{2} / 3 \nu_{\mathrm{c}}$ is the field-free diffusion coefficient and it is assumed that $\mathrm{n}$ is distributed in the mode appropriate to $\Lambda$.

Equation 13.11 leads to the solution

$$
\mathrm{n}=\mathrm{n}_{\mathrm{o}} \mathrm{e}^{-\mathrm{Dt} / \Lambda_{\mathrm{b}}^{2}}
$$

whence one obtains the average number $\mathrm{N}_{\mathrm{c}}$ of collisions suffered by an ion in diffusing out of the cavity

$$
\mathrm{N}_{\mathrm{c}}=\frac{1}{\mathrm{n}_{\mathrm{o}}} \int_{0}^{\infty} \mathrm{n} \nu_{\mathrm{c}} \mathrm{dt}=\frac{\nu_{\mathrm{c}} \Delta_{\mathrm{b}}^{2}}{\mathrm{D}}=\frac{3 \Lambda_{\mathrm{b}}^{2}}{\ell_{\mathrm{c}}^{2}}
$$

This provides a useful random-flight definition of the diffusion length $\Lambda_{\mathrm{b}}$.

14. The Einstein relation. A better definition of the diffusion coefficient can be obtained by considering a pressure gradient as a force. Equation 8.1 shows that $\mathrm{n} \mathrm{q} \overrightarrow{\mathrm{E}}$ and -grad $\mathrm{p}$ are equivalent in producing a drift velocity $\overrightarrow{\mathrm{v}}_{\mathrm{d}}$. Using the results of Sec. 12, it then follows that

$$
v_{d, i}=-\frac{\mu_{i k} \partial p}{n q} \frac{\partial}{\partial r_{k}}=-\frac{1}{n} \frac{\partial\left(D_{i k} n\right)}{\partial r_{k}}
$$

whence

$$
\mathrm{D}_{\mathrm{ik}}=\frac{\mathrm{p}}{\mathrm{nq}} \mu_{\mathrm{ik}}=\frac{\mathrm{m}}{\mathrm{q}} \frac{\mathrm{v}^{2}}{3} \mu_{\mathrm{ik}}
$$

provided $\mu_{i k}$ is not a function of position, that is, provided $\nu_{c}$, and B do not depend on position. This is frequently a good assumption in electrical discharges; then Eq. 14.1, with $\mathrm{D}$ under the derivative sign, is a suitable definition of the diffusion coefficient. In the kinetic theory of gases the gas pressure is frequently variable and then it is more suitable to put $\mathrm{D}$ outside the derivative sign. But it must be remembered that grad $p$, and not $\operatorname{grad} \mathrm{n}$, is the driving force, so that the factor $\mathrm{v}^{2} / 3$ must be retained under the derivative. 
Equation 14.2 is known as the Einstein relation, and by comparison with Eq. 12.10 it gives the diffusion tensor

$$
\mathrm{D}=\frac{v^{2}}{3}\left\|\begin{array}{ccc}
\frac{\nu_{\mathrm{c}}}{\nu_{\mathrm{c}}^{2}+\omega_{\mathrm{b}}^{2}} & \frac{\omega_{\mathrm{b}}}{\nu_{\mathrm{c}}^{2}+\omega_{\mathrm{b}}^{2}} & 0 \\
\frac{-\omega_{\mathrm{b}}}{\nu_{\mathrm{c}}^{2}+\omega_{\mathrm{b}}^{2}} & \frac{\nu_{\mathrm{c}}}{\nu_{\mathrm{c}}^{2}+\omega_{\mathrm{b}}^{2}} & 0 \\
0 & 0 & \frac{1}{\nu_{\mathrm{c}}}
\end{array}\right\|
$$

which differs from Eq. 13.8 by the addition of antisymmetric terms. These terms are lost in the random flight definition of $\mathrm{D}_{\mathrm{ik}}$ which is inherently symmetric.

Both this expression and Eq. 13.8 predict that diffusion transverse to a magnetic field decreases as $1 / \mathrm{B}^{2}$ at high fields. As far as the author is aware, this result is verified experimentally. Claims to the contrary (12) refer to conditions in which space charge has a large effect. This situation must now be considered.

15. Ambipolar diffusion. In a plasma in which there are ions of both signs, the faster ions, generally electrons, tend to diffuse out of the plasma and leave an excess of positive charge, whose space charge field $\overrightarrow{\mathrm{E}}_{\mathrm{S}}$ then retards the electrons and accelerates the slower ions, thus linking their diffusion.

Debye and Hückel (13) showed that the field of an ion in an electrolyte was reduced by a factor exp $\left(-r / \ell_{D}\right)$ as a result of shielding by the ions of opposite sign, and the theory applies equally well to the positive ions in a plasma, where

$$
l_{\mathrm{D}}^{2}=\frac{\epsilon_{\mathrm{O}} \mathrm{D}-}{\mathrm{n}_{-} \mathrm{e} \mu}
$$

If the plasma is contained in a vessel of dimensions greater than $\ell$, the ions will be able to hold their shielding electrons and the diffusion will be called ambipolar, whereas if the dimensions are much less than $\ell_{D}$, the electrons will diffuse independently of the ions, and the diffusion will be called free. These limits correspond to high and low ion concentrations, respectively. One can also consider the two limits of high and low gas concentrations in which the vessel is large or small compared with the mean free path. Allis and Rose (14) have considered the first situation, Bernstein and Holstein 
the latter (15). We shall consider the high-pressure case, since it is more closely related to the diffusion process. In this case, the flow vectors $\vec{\Gamma}_{+}$and $\vec{\Gamma}_{-}$of the positive and negative ions are given by

$$
\begin{aligned}
& \vec{\Gamma}_{+}=-\operatorname{grad} \mathrm{D}_{+} \mathrm{n}_{+}+\overrightarrow{\mathrm{E}}_{\mathrm{s}_{+}{ }_{+} \mathrm{n}_{+}} \\
& \vec{\Gamma}_{-}=-\operatorname{grad} \mathrm{D}_{-} \mathrm{n}_{-}-\overrightarrow{\mathrm{E}}_{\mathrm{s}} \mu_{-} \mathrm{n}_{-}
\end{aligned}
$$

and the space-charge field $\overrightarrow{\mathrm{E}}_{\mathrm{S}}$ is given by Poisson's equation

$$
\operatorname{div} \epsilon_{\mathrm{o}} \overrightarrow{\mathrm{E}}_{\mathrm{s}}=\left(\mathrm{n}_{+}-\mathrm{n}_{-}\right) \mathrm{e}
$$

These equations are completed by the continuity equations

$$
\begin{aligned}
& \frac{\partial \mathrm{n}_{t}}{\partial \mathrm{t}}=\mathrm{I}-\operatorname{div} \vec{\Gamma}_{+} \\
& \frac{\partial \mathrm{n}}{\partial \mathrm{t}}=\mathrm{I}-\operatorname{div} \vec{\Gamma}_{-}
\end{aligned}
$$

where $I$ is the ionization rate, and by boundary conditions. We shall consider, in particular, the stationary state in which

$$
\operatorname{div} \vec{\Gamma}_{+}=\operatorname{div} \vec{\Gamma}_{-}
$$

Equations 15.2 and 15.3 are quadratic on account of the products $\overrightarrow{\mathrm{E}}_{\mathrm{S}} \mathrm{n}$; therefore methods of solution applicable to linear equations do not apply and machine computation is necessary to obtain good solutions. However, useful results can be obtained by making two simple, though inexact, assumptions: the assumption of congruence

$$
\vec{\Gamma}_{+}=\vec{\Gamma}_{-}
$$

which is suggested, but not required, by Eq. 15.5, and the assumption of proportionality

$$
\frac{\operatorname{grad} n_{+}}{n_{+}}=\frac{\operatorname{grad} n_{-}}{n_{-}}
$$

which is true in the free limit, because then the positive and negative ions satisfy independently the same diffusion equation, and is also true in the ambipolar limit, because then the difference between $n_{+}$and $n_{-}$is very small compared with either one of these. We shall assume that $D_{+}$and $D_{-}$are constants so that they can be taken outside of the 
gradient operator. Equations 15.2 and 15.3 are then solved by first eliminating the space-charge field $\mathrm{E}_{\mathrm{S}}$ between them, obtaining

$$
\begin{aligned}
\left(\mu_{+} \mathrm{n}_{+}+\mu_{-} \mathrm{n}_{-}\right) \vec{\Gamma} & =-\mu_{+} \mathrm{n}_{+} \operatorname{grad}\left(\mathrm{D}_{-} \mathrm{n}_{-}\right)-\mu_{-} \mathrm{n}_{-} \operatorname{grad}\left(\mathrm{D}_{+} \mathrm{n}_{+}\right) \\
& =-\left(\mu_{+} \mathrm{D}_{-}+\mu_{-} \mathrm{D}_{+}\right) \mathrm{n}_{+} \operatorname{grad} \mathrm{n}-
\end{aligned}
$$

Following Schottky (16), we define the ambipolar diffusion coefficient

$$
\mathrm{D}_{\mathrm{a}}=\frac{\mu_{+} \mathrm{D}_{-}+\mu_{-} \mathrm{D}_{t}}{\mu_{+}+\mu_{-}}
$$

and setting

$$
\begin{aligned}
& \rho=\left(\mathrm{n}_{+}-\mathrm{n}_{-}\right) \mathrm{e} \\
& \sigma=\left(\mu_{+} \mathrm{n}_{+}+\mu_{-} \mathrm{n}_{-}\right) \mathrm{e}
\end{aligned}
$$

for the space-charge density and plasma conductivity, we obtain

$$
\vec{\Gamma}=-D_{a}\left(1+\mu_{-} \rho / \sigma\right) \operatorname{grad} n_{-}=-D_{s} \operatorname{grad} n_{-}
$$

where

$$
D_{S}=D_{a}\left(1+\mu_{-} \rho / \sigma\right)
$$

is an effective diffusion coefficient for electrons, and by Eq. 15.7 is not a function of position. $D_{a}$ is obviously the minimum of $D_{S}$. The space-charge field is obtained by substituting Eq. 15.11 in Eq. 15.3

$$
E_{S}=\frac{D_{S}-D_{-}}{\mu_{-}} \frac{\operatorname{grad} n_{-}}{n_{-}}
$$

and then Poisson's equation gives the charge density

$$
\rho=\epsilon_{0} \frac{D_{-}-D_{S}}{\mu_{-}}\left[\left(\frac{\operatorname{grad} n}{n}\right)^{2}-\frac{\nabla^{2} n}{n}\right]
$$

Defining the diffusion length $\Lambda$ by

$$
\Lambda^{2}=-\frac{\mathrm{n}}{\nabla^{2} \mathrm{n}}
$$


and calling "center" that point in the plasma where grad $n=0$, we find for the space charge at the center

$$
\rho_{0}=\frac{D_{-}-D_{S}}{\mu_{-}} \frac{\epsilon_{O}}{\Lambda^{2}}
$$

Obviously $D_{S}=D_{\text {- when }} \rho_{0}=0 . D_{S}$ decreases as the central space charge increases, but the space charge must saturate when $\mathrm{D}_{\mathbf{S}}=\mathrm{D}_{\mathrm{a}}$. The relation between space charge and Debye length is found by substituting from Eq. 15.1 which gives, at the center,

$$
\frac{\mathrm{n}_{+}-\mathrm{n}-}{\mathrm{n}-}=\frac{\mathrm{D}_{-}-\mathrm{D}_{\mathrm{S}}}{\mathrm{D}_{-}} \frac{\ell_{\mathrm{D}}^{2}}{\Lambda^{2}}
$$

When the Debye length equals the diffusion length, the central ion density is close to twice the electron density. As the plasma conductivity increases, the Debye length decreases, and the central ratio $n_{+} / n_{-}$tends to one. The effective diffusion coefficient $D_{s}$ can be expressed in terms of the central conductivity $\sigma_{0}$ by substituting from Eq. 15.16 in Eq. 15.12. This gives

$$
D_{S}=D_{a} \frac{D_{-}+\Lambda^{2} \sigma_{0} / \epsilon_{o}}{D_{a}+\Lambda^{2} \sigma_{0} / \epsilon_{o}}
$$

which shows clearly the two limits, $D_{-}$and $D_{a}$, for the effective diffusion coefficient as the conductivity goes from 0 to $\infty$.

We must now discuss the errors of this theory. If the proportionality assumption held rigorously, the charge density would be proportional to $\mathrm{n}$ and have a maximum at the center. However, Eq. 15.14 indicates a minimum at the center. At low conductivities the error was made in obtaining the field $\overrightarrow{\mathrm{E}}_{\mathrm{S}}$ from a small difference of large quantities $\vec{\Gamma}_{-}$and - grad D_n ; so Eq. 15.14 is false. At high conductivities one cannot conclude that $\rho$ is proportional to $n$ in taking the small difference $n_{+}-n_{-}$; so Eq. 15.14 is correct. In neither limit is the assumption of proportionality Eq. 15.7 very bad. Only in the transition is it bad, and the degree of error is shown in Fig. 8. Agreement is much better if the conductivity used in Eq. 15.18 is less than the central conductivity by a factor 5 .

The assumption of congruence follows from Eq. 15.5 when the flow is irrotational, which is true in the absence of a magnetic field. But in a magnetic field this is surely false; hence the whole derivation, including the ambipolar coefficient Eq. 15.8 must be false. Observations of diffusion in high magnetic fields indicate coefficients of the order 


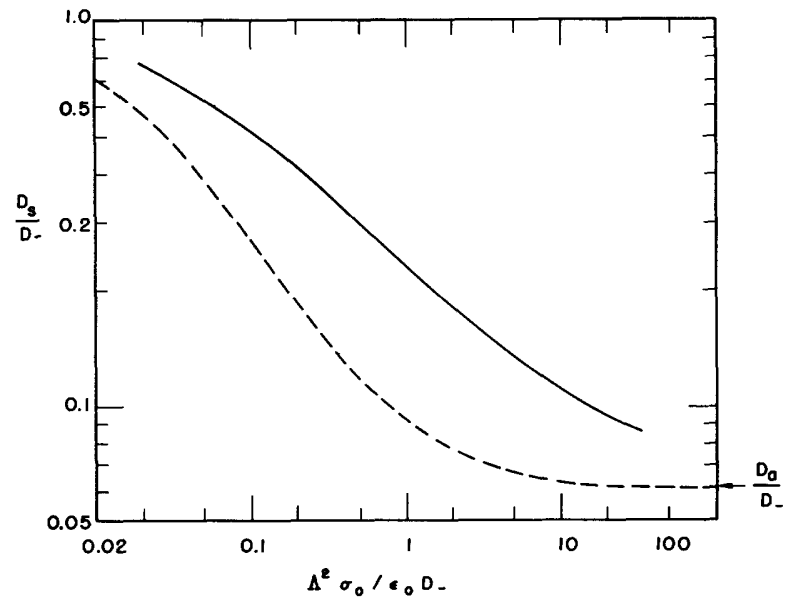

Fig. 8. Comparison of a machine solution of the combined diffusion equations with the approximate solution 15.18. The approximate solution is shown dotted. of the off-diagonal components of the diffusion tensor 14.3, and these must certainly enter an appropriate theory (17).

In a manner similar to the case of a magnetic field, one can define an effective diffusion length to take account of space charge

$$
\Lambda_{S}^{2}=\Lambda^{2} \frac{1+\Lambda^{2} \sigma_{o} / \epsilon_{o} D_{a}}{1+\Lambda^{2} \sigma_{o} / \epsilon_{o} D_{-}}
$$

and say that, to the approximation treated here, the effect of space charge on the electrons is to increase the effective size of the volume occupied by the plasma.

\section{c. Energy and Gain}

16. Elastic collisions only. In order to use the expressions derived in the preceding section, it is necessary to know the average energy of the ions. This is particularly true of the expression for the diffusion coefficient which contains $v^{2}$ explicitly, but may be true for the others when $\nu_{c}$ is a function of energy. We shall assume, however, in this section that $\nu_{c}$ and $\lambda$ are not functions of energy in order to make the integrations easier. Nothing essential is changed if they are functions of energy and the corresponding integrals can generally be performed.

The power input to the ions is obtained by multiplying the Langevin equation 11.1 by $\mathrm{m}$ v, which yields

$$
m \vec{v} \cdot \dot{\vec{v}}=m \vec{v} \cdot \vec{a}+m \vec{v} \cdot \vec{A}
$$

The term in $\vec{\omega}_{\mathrm{b}}$ has dropped out because magnetic fields do no work. Averaging over many collision times $1 / \nu_{\mathrm{c}}$, one has

$$
\overrightarrow{\mathrm{m} \overrightarrow{\mathrm{v}} \cdot \overrightarrow{\mathrm{a}}}=\overrightarrow{\mathrm{v}}_{\mathrm{g}} \cdot \mathrm{e} \overrightarrow{\mathrm{E}}=\mathrm{e} \overrightarrow{\mathrm{v}}_{\mathrm{g}} \cdot \mu^{-1} \overrightarrow{\mathrm{v}}_{\mathrm{g}}=\nu_{\mathrm{c}} \mathrm{m} \mathrm{v}_{\mathrm{g}}^{2}=2 \nu_{\mathrm{c}} \mathrm{u}_{\mathrm{d}}
$$


and making use of Eq. 11.3,

$$
\frac{d u}{d t}=\nu_{c}\left[2 u_{d}+\lambda(\bar{U}-u)\right]
$$

The first term on the right is the gain of energy from the field and we note that the ions gain twice the drift energy per collision. The second term is the loss of energy to the gas, where $\lambda$ is the fractional loss per collision. The drift energy $u_{d}$ is the rather complicated expression obtained in Sec. 12, Eq. 12.8. It can be written

$$
u_{d}=\bar{u}_{d}[1+\alpha \cos (2 \omega t+\phi)]
$$

where

$$
\begin{aligned}
\overline{\mathrm{u}}_{\mathrm{d}} & =\frac{\mathrm{e}^{2} \mathrm{E}_{\mathrm{p}}^{2}}{4 \mathrm{~m}\left[\nu_{\mathrm{c}}^{2}+\left(\omega-\omega_{\mathrm{b}}\right)^{2}\right]\left[\nu_{\mathrm{c}}^{2}+\left(\omega+\omega_{\mathrm{b}}\right)^{2}\right]} \\
\alpha & =\frac{\sqrt{\left[\nu_{\mathrm{c}}^{2}+\left(\omega-\omega_{\mathrm{b}}\right)^{2}\right]\left[\nu_{\mathrm{c}}^{2}+\left(\omega+\omega_{\mathrm{b}}\right)^{2}\right]}}{\nu_{\mathrm{c}}^{2}+\omega^{2}+\omega_{\mathrm{b}}^{2}} \\
\tan \phi & =\frac{2 \nu_{\mathrm{c}}^{2} \omega}{\nu_{\mathrm{c}}^{2}-\omega^{2}+\omega_{\mathrm{b}}^{2}}
\end{aligned}
$$

$\alpha$ is always less than one except when there is no magnetic field, or in the dc case. Substituting in Eq. 16.2, and assuming that $\lambda$ and $\nu_{c}$ are not functions of the energy, we obtain, for the random energy

$$
\mathrm{u}=\overline{\mathrm{U}}+\frac{2 \overline{\mathrm{u}}_{\mathrm{d}}}{\lambda}\left[1+\frac{\alpha \cos \left(2 \omega \mathrm{t}+\phi^{\prime}\right)}{\sqrt{1+\left(2 \omega / \lambda \nu_{\mathrm{c}}\right)^{2}}}\right]+\mathrm{A} \mathrm{e}^{-\lambda \nu_{\mathrm{c}} \mathrm{t}}
$$

The ion energy consists of three terms. The first is the mean gas energy required for thermal equilibrium. The second depends on the field. At high frequencies $\omega / \nu_{\mathrm{c}} \gg \lambda / 2$, it is substantially constant with a slight alternating ripple. At low frequencies $\omega / \nu_{\mathrm{c}} \ll \lambda / 2$, it follows $\mathrm{E}^{2}$ and is completely modulated. The third term is a transient and gives the time constant $1 /\left(\lambda \nu_{c}\right)$ at which the energy approaches its equilibrium value.

A difficulty might have been expected at low pressures when there are many oscillations per collision, since the average taken in the Langevin equation then covers many 
oscillations. However, in this case $2 \omega / \lambda \nu_{c}$ is very large and the energy is not modulated so that it makes no difference.

In either the high frequency $\left(\omega \gg \lambda v_{c} / 2\right)$ or the dc case, the energy eventually reaches an equilibrium value

$$
u_{e}=\bar{U}+2 \bar{u}_{d} / \lambda
$$

17. Energy gain and inelastic collisions. Whereas the energy transfers by an electron to recoil in an elastic collision, or to rotation and vibration of a molecule, are always small compared with the electron's energy $u$, and, therefore, can be treated, as they are in Eq. 16.2, as continuous, the losses to electronic excitation are quite otherwise. The probability of excitation of any level has a maximum not far above the excitation potential of that level, so that, no matter what the energy $u$ of an electron may be, provided that it is above the lowest excitation energy $\mathrm{eV}_{\mathrm{x}}$, the chances are it will excite a level not far below $u$ and therefore be left with a small energy $u_{q}$. The life of an electron, therefore, consists of a succession of periods in which the energy rises from $u_{q}$ to $u_{x}$, followed by sudden drops back to $u_{q}$. After $N_{x}$ such rises, the electron may ionize an atom, producing a new electron, or may disappear through diffusion to the walls or attachment to a molecule, the probabilities of ionization and disappearance being equal in the steady state.

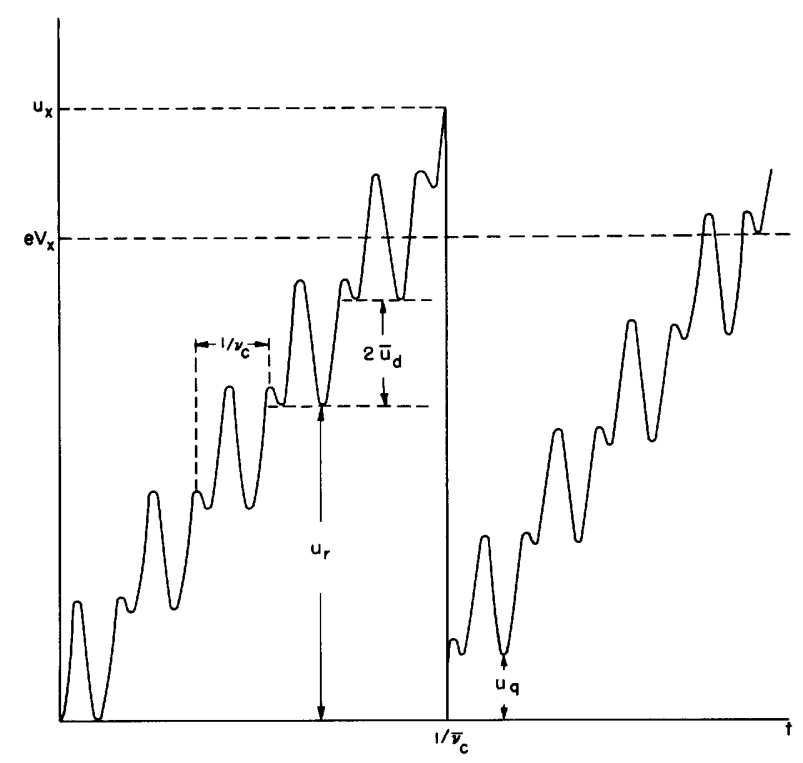

Fig. 9. Energy of the average electron as a function of the time.
We shall consider here the case of disappearance by diffusion, and shall neglect the small energy losses to recoil, rotation, and vibration.

To render these ideas quantitative, consider a group of $\mathrm{n}$ electrons of the same energy u. Their energy is increasing at the rate

$$
\frac{\mathrm{d} \mathrm{u}}{\mathrm{d} \mathrm{t}}=2 \nu_{\mathrm{c}} \mathrm{u}_{\mathrm{d}}
$$

At the same time, the number $\mathrm{n}$ is being depleted by diffusion at the rate determined by Eq. 13.13

$$
\frac{\mathrm{d} n}{\mathrm{~d} t}=-\mathrm{n} \nu \frac{\mathrm{l}_{\mathrm{c}}^{2}}{3 \Lambda^{2}}
$$


The time can be eliminated between these equations to give

$$
\frac{\mathrm{dn}}{\mathrm{n}}=-\frac{l_{\mathrm{c}}^{2}}{3 \Lambda^{2}} \frac{\mathrm{du}}{2 \mathrm{u}_{\mathrm{d}}}=-\frac{\frac{4}{3}}{\left(\mathrm{e} \mathrm{E}_{\mathrm{p}} \Lambda\right)^{2}} \frac{\left[\nu_{\mathrm{c}}^{2}+\left(\omega-\omega_{\mathrm{b}}\right)^{2}\right]\left[\nu_{\mathrm{c}}^{2}+\left(\omega+\omega_{\mathrm{b}}\right)^{2}\right]}{\nu_{\mathrm{c}}^{2}\left(\nu_{\mathrm{c}}^{2}+\omega^{2}+\omega_{\mathrm{b}}^{2}\right)} \mathrm{u} \mathrm{du}
$$

This expression can be integrated if $\nu_{c}$ is any known function of $u$. Assuming a constant collision frequency, the integral is

$$
\ln \frac{\mathrm{n}_{\mathrm{q}}}{\mathrm{n}_{\mathrm{x}}}=\frac{\left[\nu_{\mathrm{c}}^{2}+\left(\omega-\omega_{\mathrm{b}}\right)^{2}\right]\left[\nu_{\mathrm{c}}^{2}+\left(\omega+\omega_{\mathrm{b}}\right)^{2}\right]}{\nu_{\mathrm{c}}^{2}\left(\nu_{\mathrm{c}}^{2}+\omega^{2}+\omega_{\mathrm{b}}^{2}\right)} \frac{2}{3} \frac{\mathrm{u}_{\mathrm{x}}^{2}-\mathrm{u}_{\mathrm{q}}^{2}}{\left(\mathrm{e} \mathrm{E}_{\mathrm{p}} \Lambda\right)^{2}}
$$

If every excitation were an ionization two electrons would start out at $u_{q}$ for every one which reached $u_{x}$ and one would have $n_{q} / n_{x}=2$, but if $N_{x}$ successive periods of gain are required to ionize

$$
\frac{\mathrm{n}_{\mathrm{q}}}{\mathrm{n}_{\mathrm{x}}}=1+\frac{1}{\mathrm{~N}_{\mathrm{x}}}
$$

and if the number of excitations per ionization, $\mathrm{N}_{\mathrm{x}}$, is large, as it generally is, one has

$$
\frac{\left(\mathrm{E}_{\mathrm{p}} \Lambda\right)^{2}}{2} \frac{\nu_{\mathrm{c}}^{2}\left(\nu_{\mathrm{c}}^{2}+\omega^{2}+\omega_{\mathrm{b}}^{2}\right)}{\left[\nu_{\mathrm{c}}^{2}+\left(\omega-\omega_{\mathrm{b}}\right)^{2}\right]\left[\nu_{\mathrm{c}}^{2}+\left(\omega+\omega_{\mathrm{b}}\right)^{2}\right]}=\mathrm{N}_{\mathrm{x}} \frac{\mathrm{u}_{\mathrm{x}}^{2}-\mathrm{u}_{\mathrm{q}}^{2}}{3 \mathrm{e}^{2}}
$$

This formula has a simple interpretation. $\mathrm{E}_{\mathrm{p}} \Lambda$ is in effect the voltage applied to the plasma. The factor $1 / 2$ reduces it to an $\mathrm{rms}$ value, and the factor containing $\omega$ is a power factor arising from the phase of the electron's motion, but also containing a resonance denominator which enhances the field when the applied frequency equals the cyclotron frequency (18). One can combine them to define an "effective" field $E_{\text {ef }}$ by

$$
\mathrm{E}_{\mathrm{ef}}^{2}=\frac{\mathrm{E}_{\mathrm{p}}^{2}}{2\left[\nu_{\mathrm{c}}^{2}+\left(\omega-\omega_{\mathrm{b}}\right)^{2}\right]\left[\nu_{\mathrm{c}}^{2}+\left(\omega+\omega_{\mathrm{b}}\right)^{2}\right]}
$$

or by the corresponding integral expression, if $\nu$ is a function of the energy. The power factor is 1 at higher pressures so that $\nu_{c}^{2}>\omega^{2}+\omega_{b}^{2}$ no matter what the energy dependence of $\nu_{c}$. The right-hand side of Eq. 17.6 is a geometric mean between the total energy $N_{x}\left(u_{x}-u_{q}\right) / e$ (in electron volts) an electron must gain to ionize and a "mean" energy $\left(u_{x}+u_{q}\right) / 3 e$, which corresponds to the energy lost to diffusion. 
Equation 17.6 can now be written

$$
\left(E_{e f} \Lambda\right)^{2}=N_{x} \frac{u_{x}^{2}-u_{q}^{2}}{3 e^{2}}
$$

and it determines the field at which ionization balances diffusion, a condition termed by Langmuir and Tonks (19) "plasma balance." It determines the high frequency breakdown field in a cavity if one uses the diffusion length $\Lambda$ or $\Lambda_{b}$, or it determines the operating field in an active discharge, either ac or dc, if one uses $\Lambda_{\mathrm{s}}$. It is limited, however, to values of $\mathrm{E} / \mathrm{p}$ sufficiently large that recoil losses are, in fact, negligible.

Present knowledge of the excitation functions of individual levels is not sufficient to determine $u_{q}$, but it is of the order of one or two volts, and its square is negligible. The average excitation energy $u_{x}$ is calculable if the total probability of excitation of all levels $\mathrm{P}_{\mathrm{x}}$ is known. A reasonable assumption is that the ratio of inelastic to total collision probability varies linearly with the energy above $\mathrm{eV}_{\mathrm{x}}$

$$
P_{x}=P_{c} h_{x}\left(u-e V_{x}\right)
$$

If Eq. 17.2 is extended to energies above excitation, it becomes

$$
\frac{\mathrm{dn}}{\mathrm{dt}}=-\mathrm{n}_{\nu_{\mathrm{c}}}\left[\frac{\ell_{\mathrm{c}}^{2}}{3 \Lambda^{2}}+\mathrm{h}_{\mathrm{x}}(\mathrm{u}-\mathrm{eV})\right]
$$

and the diffusion term, $e_{\mathrm{c}}^{2} / 3 \Lambda^{2}$, can generally be neglected compared with the excitation term in this range. Combining this with Eq. 17.1 and assuming $u_{d}$ constant, yields, for the number of electrons which reach the energy $u$,

$$
n=n_{x} \exp \left[-\frac{h_{x}}{4 u_{d}}\left(u-e v_{x}\right)^{2}\right]
$$

and the average excitation energy is

$$
u_{x}=\frac{1}{n_{x}} \int_{e V_{x}}^{\infty} u d n=e v_{x}+\sqrt{\frac{\pi u_{d}}{h_{x}}}=e V_{x}+c \frac{E}{p}
$$

The average electron will suffer an inelastic collision when it has exceeded the lowest excitation potential by an amount proportional to $E / p$. This overshoot is normally of the order of a volt, but becomes large at low pressures. 
A similar calculation applied to the excitation number $\mathrm{N}_{\mathrm{x}}$ yields

$$
N_{x}=\frac{n_{x}}{n_{i}}=\frac{h_{x}}{n_{i}} 4 x^{2} e^{x^{2}}
$$

where

$$
x=\sqrt{\frac{h_{x}}{u_{d}}} \frac{e V_{i}-e V_{x}}{2} \approx \frac{p}{E}
$$

but, since $\mathrm{N}_{\mathrm{x}}$ is often very large, the approximations of this section are not adequate and we must refer to Eq. 33.8 .

The approximation made in this section of neglecting recoil losses Eq. 17.1 could be corrected, but it is useless to do so because of inherent errors in the method that is used. This is particularly obvious in calculating infrequent processes such as ionization. The equilibrium energy of an electron without inelastic collisions is

$$
u_{e}=\bar{U}+2 \bar{u}_{d} / \lambda
$$

and substitution of experimental values of $\mathrm{E} / \mathrm{p}$ for high-frequency breakdown at higher pressures often shows $u_{e}$ to be below the ionization potential, and even below the excitation potential. In these cases the "average electron" never ionizes. It is only the exceptional electron that is able to ionize, and one must know the distribution of electrons about the average. The proper way to calculate this is by the Boltzmann equation, to which we now turn. 


\section{THE BOLTZMANN EQUATION}

\section{a. The Derivative Terms}

18. Continuity in phase space. We now abandon the attempt to follow the motion of an individual electron and treat instead the changes in an assembly of electrons defined by giving the number $d^{6} \mathrm{~N}$ of electrons in a volume $d^{3} \mathrm{r}$ of configuration space and $\mathrm{d}^{3} \mathrm{v}$ of velocity space. This number defines a distribution function $f(\vec{r}, \vec{v}, t)$ by

$$
d^{6} N=f d^{3} r d^{3} v
$$

It also defines a particle current in phase space whose component in configuration space is given by

$$
\vec{\gamma}=\overrightarrow{v f}
$$

and whose component in velocity space is determined by the applied forces

$$
\vec{g}=\left(\vec{a}+\overrightarrow{\omega_{b}} \times \vec{v}\right) f
$$

For continuous forces one would have the continuity equation

$$
\frac{\partial f}{\partial t}+\operatorname{div}_{r} \vec{\gamma}+\operatorname{div}_{v} \vec{g}=0
$$

but collisions have the effect of removing an electron from one element of velocity space and replacing it in another, or even creating a new one in the case of ionization. One provides for this by writing

$$
\frac{\partial f}{\partial t}+\operatorname{div}_{r} \vec{\gamma}+\operatorname{div}_{v} \vec{g}=B(t)
$$

where $B$ is an integral to be discussed in the next section. This is the Boltzmann equation.

19. Expansion in spherical harmonics. Except for the singular case of a constant mean free time, it is always necessary to expand the function $f$, and there are two convenient expansions. When the system considered is close to thermal equilibrium, departures from equilibrium being caused by some agent $\alpha$, the distribution function can be expanded in powers of $\alpha$

$$
\mathrm{f}=\mathrm{f}^{\mathrm{o}}+\alpha \mathrm{f}^{1}+\alpha^{2} \mathrm{f}^{2} \ldots
$$


This was the method by which Enskog solved Boltzmann's equation; it is highly developed by Chapman and Cowling (20). It does not converge well for charged particles in an electric field. In all other cases the distribution function is expanded in spherical harmonics in velocity space

$$
f(\vec{r}, \vec{v})=\sum_{\ell} f^{\ell}(\vec{r}, v) P_{\ell}(\cos \theta)=f^{O}+f^{1} \cos \theta+\ldots
$$

where, if necessary, one can also use the associated Legendre functions $\mathrm{P}_{\ell}^{\mathrm{m}}$. We shall assume that this series converges sufficiently rapidly for the first two terms to suffice, but we shall not assume that $f^{\circ}$ is Maxwellian, as this is generally far from true when any appreciable electric field is present.

Because of the orthogonality of the spherical harmonics one has, for any scalar function $s(v)$,

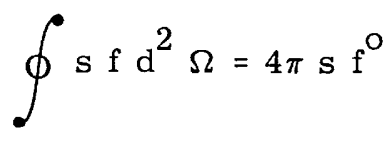

and for the vector $\vec{v}$

$$
\oint \overrightarrow{\mathrm{v}} \mathrm{fd} \mathrm{d}^{2} \Omega=\frac{4 \pi}{3} \mathrm{v} \overrightarrow{\mathrm{f}}^{1}
$$

where the vector $\overrightarrow{\mathrm{f}}^{-1}$ is directed along the polar axis of $\mathrm{P}_{1}$.

The two scalars of greatest interest are the electron concentration

$$
\mathrm{n}=\int_{0}^{\infty} \mathrm{f}^{\mathrm{o}} 4 \pi \mathrm{v}^{2} \mathrm{dv}
$$

and the energy density is

$$
n \bar{u}=\int_{0}^{\infty} u f^{O} 4 \pi v^{2} d v
$$

and there are two corresponding vector integrals: the particle flow in configuration space is 


$$
\vec{\Gamma}=n \vec{v}_{d}=\int \vec{f}^{1} \frac{4 \pi}{3} v^{3} d v
$$

and the total radial flow in velocity space is

$$
G=\oint \vec{g} \cdot \vec{v} v d^{2} \Omega=\frac{4 \pi}{3} v^{2} \vec{a} \cdot \vec{f}^{1}
$$

The quantity $G$ represents the rate of flow of electrons across any energy value in the direction of increasing energy. It deserves a convenient name and we shall call it the "gain".

We shall now introduce the expansion 19.2 in the Boltzmann equation and expand each term separately. Three relations between spherical harmonics are useful

$$
\begin{aligned}
& (2 \ell+1) \mathrm{P}_{\ell} \cos \theta=(\ell+1) \mathrm{P}_{\ell+1}+\ell \mathrm{P}_{\ell-1} \\
& (2 \ell+1) \sin ^{2} \theta \frac{\partial \mathrm{P}_{\ell}}{\partial \cos \theta}=\ell(\ell+1)\left(\mathrm{P}_{\ell-1}-\mathrm{P}_{\ell+1}\right) \\
& (2 \ell+1) \oint \mathrm{P}_{\ell} \mathrm{P}_{\mathrm{m}} \mathrm{d}^{2} \Omega=4 \pi \delta_{\ell \mathrm{m}}
\end{aligned}
$$

20. The gradient term. Taking the polar axis along the direction of grad $\mathrm{n}$ and assuming that there is rotational symmetry about that axis so that the associated Legendre polynomials are not required,

$$
\begin{aligned}
\operatorname{div}\left(\vec{v} f^{\ell} P_{\ell}\right) & =\vec{v} \cdot\left(\operatorname{grad} f^{\ell}\right) P_{\ell}=v \frac{\partial f^{\ell}}{\partial z} \cos \theta P_{\ell} \\
& =v \frac{\partial f^{\ell}}{\partial z} \frac{P_{\ell-1}+(\ell+1) P_{\ell+1}}{2 \ell+1}
\end{aligned}
$$

so that 


$$
\begin{aligned}
\operatorname{div}(\overrightarrow{\mathrm{v}} \mathrm{f}) & =\mathrm{v} \sum_{0}^{\infty} \frac{\partial}{\partial \mathrm{z}}\left[\frac{\ell}{2 \ell+1} \mathrm{f}^{\ell-1}+\frac{\ell+1}{2 \ell+3} \mathrm{f}^{\ell+1}\right] \mathrm{P}_{\ell} \\
& =\frac{\mathrm{v}}{3} \frac{\partial \mathrm{f}^{1}}{\partial \mathrm{z}}+\mathrm{v} \cos \theta \frac{\partial}{\partial \mathrm{z}}\left[\mathrm{f}^{\mathrm{O}}+\frac{2}{5} \mathrm{f}^{2}\right]+\ldots \\
& =\frac{\mathrm{v}}{3} \operatorname{div} \overrightarrow{\mathrm{f}}^{1}+\overrightarrow{\mathrm{v}} \cdot \operatorname{grad}\left[\mathrm{f}^{\mathrm{O}}+\frac{2}{5} \mathrm{f}^{2}\right]+\ldots
\end{aligned}
$$

In the last line the scalar function $f^{1}$ has been replaced by the fector $\vec{f}^{1}$, and this notation removes the initial restriction on the direction of the polar axis of $\mathrm{P}_{1}$.

21. Electric terms. In this section we take the polar axis of the spherical harmonics along the electric field $\vec{E}$. Using the expression for partial derivatives,

$$
\left(\frac{\partial}{\partial v_{z}}\right)_{v_{x} v_{y}}=\cos \theta\left(\frac{\partial}{\partial v}\right)_{\theta, \phi}+\frac{\sin ^{2} \theta}{v}\left(\frac{\partial}{\partial \cos \theta}\right)_{v, \phi}
$$

we find

$$
\begin{aligned}
\operatorname{div}_{\mathrm{v}} \overrightarrow{\mathrm{a}} \mathrm{f}^{\ell} \mathrm{P}_{\ell} & =\overrightarrow{\mathrm{a}} \cdot \operatorname{grad} \mathrm{f}^{\ell} \mathrm{P}_{\ell}=\mathrm{a} \cos \theta \frac{\partial \mathrm{f}^{\ell}}{\partial \mathrm{v}} \mathrm{P}_{\ell}+\frac{\mathrm{af}}{\mathrm{v}} \sin ^{2} \theta \frac{\partial \mathrm{P}_{\ell}}{\partial \cos \theta} \\
\operatorname{div}_{\mathrm{v}} \overrightarrow{\mathrm{a}} \mathrm{f} & =\mathrm{a} \sum\left[\frac{\ell}{2 \ell-1} \mathrm{v}^{\ell-1} \frac{\mathrm{d}}{\mathrm{dv}} \frac{\mathrm{f}^{\ell-1}}{\mathrm{v}-1}+\frac{\ell+1}{2 \ell+3} \frac{1}{\mathrm{v}^{\ell+2}} \frac{\mathrm{d}}{\mathrm{dv}} \mathrm{v}^{\ell+2} \mathrm{f}^{\ell+1}\right] \mathrm{P}_{\ell} \\
& =\mathrm{a}\left[\frac{1}{3 \mathrm{v}^{2}} \frac{\mathrm{d}}{\mathrm{dv}} \mathrm{v}^{2} \mathrm{f}^{1}+\left(\frac{\mathrm{df}}{\mathrm{dv}}+\frac{2}{5} \frac{1}{3} \frac{\mathrm{d}}{\mathrm{dv}} \mathrm{v}^{3} \mathrm{f}^{2}\right) \cos \theta+\cdots\right. \\
& =\frac{1}{3 \mathrm{v}^{2}} \frac{\mathrm{d}}{\mathrm{dv}} \mathrm{v}^{2} \overrightarrow{\mathrm{a}} \cdot \overrightarrow{\mathrm{f}}^{1}+\overrightarrow{\mathrm{a}} \cdot \overrightarrow{\mathrm{v}}\left(\frac{1}{\mathrm{v}} \frac{\mathrm{df}}{\mathrm{dv}}+\frac{2}{5 \mathrm{v}} \frac{\mathrm{d}}{\mathrm{dv}} \mathrm{v}^{3} \mathrm{f}^{2}\right)+\cdots
\end{aligned}
$$

in which we have again introduced the vector notation to eliminate the requirement on the polar axis $\mathrm{P}_{1}$.

22. Magnetic terms. Since

$$
\operatorname{div}_{v}\left(\overrightarrow{\omega_{b}} \times \vec{v}\right)=0
$$

we have 


$$
\operatorname{div}_{v}\left(\overrightarrow{\omega_{b}} \times \overrightarrow{v f}\right)=\left(\vec{\omega}_{b} \times \vec{v}\right) \cdot \operatorname{gradf}_{v}^{f}=\vec{v} \cdot\left(\vec{\omega}_{b} \times \operatorname{grad} f_{v}\right)
$$

The expansion involves the associated Legendre functions and the general term will not be given. The first two terms yield

$$
\operatorname{div}_{v}\left(\vec{\omega}_{b} \times \overrightarrow{v f}\right)=-\vec{v} \cdot\left(\vec{\omega}_{b} \times \vec{f}^{1}\right) / v
$$

23. Expansion in Fourier series. We shall assume an alternating electric field, $\mathrm{E} \sim \mathrm{e}^{\mathrm{j} \omega \mathrm{t}}$, since we can always obtain the direct-current formulae by setting $\omega=0$ and replacing root-mean-square values by the dc values. The distribution function will then, in general, be a Fourier series in $\omega t$ :

$$
f=\sum_{\ell} \sum_{m} f_{m}^{\ell} P_{\ell} e^{m j \omega t}
$$

and

$$
\frac{\partial f}{\partial t}=\sum_{\ell} \sum_{m} m j \omega f_{m}^{\ell} P_{\ell} e^{m j \omega t}
$$

The exponential notation is very convenient for all linear equations. Unfortunately, we shall have to take products such as $\vec{a} f^{\ell}$ and the meaning of such a product is the product of the real parts and not the real part of the product. Performing this operation and then reintroducing the exponential notation, we have

$$
2 a f^{\ell}=a\left[f_{1 r}^{\ell}+\left(2 f_{0}^{\ell}+f_{2}^{\ell}\right) e^{j \omega t}+\sum_{m=2}^{\infty}\left(f_{m-1}^{\ell}+f_{m+1}^{\ell}\right) e^{m j \omega t}\right]
$$

where $\mathrm{f}_{1 \mathrm{r}}^{\ell}$ indicates the real part of $\mathrm{f}_{1}^{\ell}$.

24. The component equations. Substituting from Secs. 20 to 23 in Eq. 18.4 and decomposing the Boltzmann integral term $\mathrm{B}$ as well, one obtains an infinite set of equations of which the zero- and first-order ones are given below:

$$
\begin{aligned}
& B_{o}^{O}=\frac{v}{3} \operatorname{div} \vec{f}_{o}^{1}+\frac{1}{6 v^{2}} \frac{d}{d v}\left(v^{2} \vec{a} \cdot \vec{f}_{1 r}^{1}\right) \\
& B_{1}^{O}=j \omega f_{1}^{O}+\frac{v}{3} \operatorname{div} \vec{f}_{1}^{1}+\frac{1}{6 v^{2}} \frac{d}{d v}\left[v^{2} \vec{a} \cdot\left(2 \vec{f}_{o}^{1}+\vec{f}_{2}^{1}\right)\right]
\end{aligned}
$$




$$
\begin{aligned}
& \vec{B}_{o}^{1}=v \operatorname{grad}\left(f_{o}^{o}+\frac{2}{5} f_{o}^{2}\right)+\vec{a}\left[\frac{1}{2} \frac{d f^{o}}{d v}+\frac{1}{5 v} \frac{d}{d v}\left(v^{3} f_{1 r}^{2}\right)\right]-\vec{\omega}_{b} \times \vec{f}_{o}^{1} \\
& \vec{B}_{1}^{1}=j \omega \vec{f}_{1}^{1}+v \operatorname{grad}\left(f_{1}^{o}+\frac{2}{5} f_{1}^{2}\right)+ \\
& \left.+\vec{a}\left[\frac{1}{2} \frac{d}{d v}\left(2 f_{o}^{o}+f_{2}^{o}\right)+\frac{1}{5 v^{3}} \frac{d}{d v} v^{3}\left(2 f_{o}^{2}+f_{2}^{2}\right)\right]-\vec{w}_{b} \times \vec{f}_{1}^{1}\right\}
\end{aligned}
$$

In the case of electrons (but not ions) the spherical harmonic expansion converges well and the terms in $\mathrm{f}^{2}$ are negligible. The Fourier series also converges well at high frequencies $\left(\omega / \nu_{c}>\lambda / 2\right)$ (Sec。16). The transition to low frequencies in which the second-harmonic terms become large has been studied by Margenau (21); this case will not be considered here. We, therefore, simplify the above set of equations to

$$
\begin{aligned}
& B_{o}^{O}=\frac{v}{3} \operatorname{div} \vec{f}_{o}^{1}+\frac{1}{6 v^{2}} \frac{d}{d v}\left(v^{2} \vec{a} \cdot \vec{f}_{1 r}^{1}\right) \\
& B_{1}^{O}=j \omega f_{1}^{O}+\frac{v}{3} \operatorname{div} \vec{f}_{1}^{1}+\frac{1}{3 v^{2}} \frac{d}{d v}\left(v^{2} \vec{a} \cdot \vec{f}_{o}^{1}\right) \\
& \vec{B}_{o}^{1}=v \operatorname{grad} f_{o}^{O}+\frac{\vec{a}}{2} \frac{d f^{o}}{d v}-\vec{\omega}_{b} \times \vec{f}_{o}^{1} \\
& \vec{B}_{1}^{1}=j \omega \vec{f}_{1}^{1}+v \operatorname{grad} f_{1}^{o}+\vec{a}_{\frac{o}{d v}}-\vec{\omega}_{b} \times \vec{f}_{1}^{1}
\end{aligned}
$$

Some insight into these equations is gained by neglecting the collision terms and then multiplying Eq. 24.5 and Eq. 24.6 by $4 \pi \mathrm{v}^{2} \mathrm{dv}$ and integrating. Equation 24.5 gives

$$
\operatorname{div} \vec{\Gamma}(\mathrm{u})=\operatorname{div} \int_{\mathrm{v}}^{\infty} \overrightarrow{\mathrm{f}}_{\mathrm{o}}^{1} \frac{4 \pi}{3} \mathrm{v}^{3} \mathrm{dv}=\frac{2 \pi}{3} \mathrm{v}^{2} \overrightarrow{\mathrm{a}} \cdot \overrightarrow{\mathrm{f}}_{1 \mathrm{r}}^{1}=\mathrm{G}
$$

From Eq. 19.7 we see that the left side is the divergence of the flow of particles whose velocity is above v. On the right we have, from Eq. 19.8, noting that a factor $1 / 2$ comes from the average of $\cos ^{2} \omega t$, the gain through the sphere radius v. Equation 24.9, therefore, expresses the conservation of particles whose speed is above v. Extending the integral down to zero yields 


$$
\operatorname{div} \vec{\Gamma}_{0}=0
$$

and the similar integration of Eq. 24.6 yields

$$
\operatorname{div} \vec{\Gamma}_{1}=-j \omega \vec{n}_{1}
$$

The divergence of the alternating flow $\vec{\Gamma}_{1}$ produces an alternating electron density $n_{1}$. The alternating flow $\vec{\Gamma}_{1}$ is driven by $\vec{a} n_{0}$ and, therefore, $n_{1}$ exists by virtue of a divergence in $\vec{a} n_{0}$. As $\vec{a}$ is divergenceless, $n_{1}$ is produced by the interaction of an alternating electric field and a concentration gradient in the same direction. Such interactions may well occur, but we shall study situations designed to minimize them. It will be assumed that $n_{1}$ and $f_{1}^{o}$ can be neglected. The four equations then reduce to three simpler equations

$$
\begin{aligned}
& B_{o}^{o}=\frac{v}{3} \operatorname{div} \vec{f}_{o}^{1}+\frac{1}{6 v} \frac{d}{d v}\left(v^{2} \vec{a} \cdot \vec{f}_{1 r}^{1}\right) \\
& \vec{B}_{o}^{1}=v \operatorname{grad} f_{o}^{o}-\vec{\omega}_{b} \times \vec{f}_{o}^{1} \\
& \vec{B}_{1}^{1}=j \omega \vec{f}_{1}^{1}+\vec{a} \frac{d f_{0}^{o}}{d v}-\vec{\omega}_{b} \times \vec{f}_{1}^{1}
\end{aligned}
$$

We must now evaluate the collision integral $B$, and its components $\mathrm{B}_{\mathrm{m}}^{\ell}$, defined by

$$
\mathrm{B}=\sum \mathrm{B}_{\mathrm{m}}^{\ell} \mathrm{P}_{\ell} \mathrm{e}^{\mathrm{mj} \omega \mathrm{t}}
$$

\section{b. The Collision Integral}

25. Geometry of a collision. Consider a collision between an electron, denoted by lower case letters, and a neutral molecule, denoted by capital letters. In a collision, the positions of these particles do not change appreciably but their velocities change; we shall denote by primes the velocities of particles about to be scattered into $d^{3} v d^{3} v$ about the velocities $\vec{v}$ and $\vec{v}$. We do not consider the changing velocities during the collision but only the velocities before and after the particles are within range of their interaction forces. During the collision the velocity of the center of gravity, $\vec{v}_{g}$, remains fixed and if the collision was elastic, the relative velocity $c$ has the same magnitude before and after but has changed in direction by an angle $\chi_{g}$, called the scattering angle in the center of gravity system. The center of gravity divides the relative velocity 


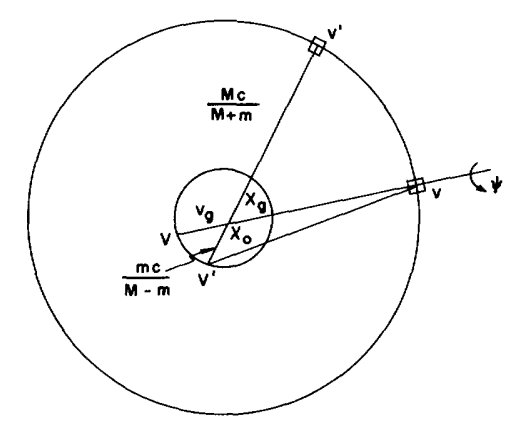

Fig. 10. Geometry of a collision in velocity space. The circles represent spheres. If the molecule is at rest before the collision, the origin is at $\mathrm{V}^{\prime}$.

inversely as the masses, so that the velocities of electron and molecule remain on two spheres in velocity space centered on the velocity of the center of gravity and of radii $\mathrm{Mc} /(\mathrm{M}+\mathrm{m})$ and $\mathrm{mc} /(\mathrm{M}+\overrightarrow{\mathrm{m}})$. The radius of the molecule's sphere is very small compared with that of the electron, so that it can almost be considered as a point. If the molecule was at rest before the collision, the origin of velocity coordinates should be taken at $\vec{V}$ ' and the electronic velocities before and after the collision are given by the lines $V^{\prime} v^{\prime}$ and $\mathrm{V}^{\prime} \mathrm{v}$ of Fig. 10. In the triangle, $\overrightarrow{\mathrm{V}}^{\prime}, \overrightarrow{\mathrm{v}}_{\mathrm{g}}, \overrightarrow{\mathrm{v}}$, we have

$$
\left(\vec{v}-\vec{V}^{\prime}\right)^{2}=c^{2}-\frac{2 M m}{(M+m)^{2}} c^{2}\left(1-\cos x_{g}\right)
$$

or if $\mathrm{V}^{\prime}=0$

$$
v^{\prime}-v^{2}=\frac{2 M m}{(M+m)^{2}} \quad v^{\prime 2}\left(1-\cos x_{g}\right)
$$

Equation 25.2 gives the energy loss by an electron in striking a molecule at rest. This energy goes into the recoil energy of the molecule. For electrons it is small and we can write it in the form

$$
-\frac{\Delta v}{v}=\frac{M m}{(M+m)^{2}}\left(1-\cos x_{g}\right)
$$

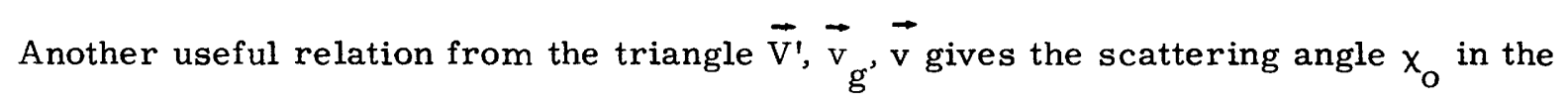
laboratory system in terms of the scattering angle $x_{g}$ in the center of gravity system. It is

$$
\sin ^{2} x_{0}=\frac{M^{2} \sin ^{2} x_{g}}{M^{2}+m^{2}+2 M m \cos x_{g}}
$$


The scattering function $\sigma(x)$ is deduced from the force law as a function of $x_{g}$ and c, and is measured experimentally in terms of $x_{0}$ and $v^{\prime}$, and it is always necessary to reduce one to the other. The dependence of $\sigma$ on the azimuthal angle $\psi$ is never known and it will be assumed that $\sigma$ does not depend on $\psi$. The combination c $\sigma$ always enters the equations and we shall use the symbol $\rho(c)=c \sigma$ for it ( $\rho$ has the dimensions of a volume per second per steradian).

26. Collision integrals. The rate of change of the number of particles in a velocity volume element $d^{3} \mathrm{v}$ is given by taking the difference between the number of particles scattered into that volume element and the number scattered out

$$
B d^{3} v=\int F\left(\vec{V}^{\prime}\right) f\left(\vec{v}^{\prime}\right) \rho d^{2} \Omega d^{3} V^{\prime} d^{3} v^{\prime}-\int F(\vec{V}) f(\vec{v}) \rho d^{2} \Omega d^{3} V d^{3} v
$$

$\rho$ is the same in both these integrals and $\mathrm{d}^{2} \Omega$ is the solid angle of the scattering sphere. $F\left(\vec{V}^{\prime}\right) d^{3} V^{\prime}$ and $f\left(\vec{v}^{\prime}\right) d^{3} v^{\prime}$ are the numbers of particles in position to scatter into the volume elements $d^{3} v$ and $d^{3} v$, and the integrals are to be taken over all scattering angles and all molecular velocities. By Liouville's theorem

$$
d^{3} v^{\prime} d^{3} v^{\prime}=d^{3} v d^{3} v
$$

so that the two integrals may be combined into one

$$
B=\int\left[F\left(\vec{V}^{\prime}\right) f\left(\vec{v}^{\prime}\right)-F(\vec{V}) f(\vec{v})\right] \rho d^{2} \Omega d^{3} V
$$

which is the conventional way of writing Boltzmann's collision integral. This form is, however, rarely convenient, since $F\left(V^{\prime}\right)$ contains $V^{\prime}$ and not $V$ and it is not easy to integrate it with respect to $V$. It is more convenient to keep dV' as in Eq. 26.1 and convert $d^{3} v^{\prime}$ into $d^{3} v$. Referring to Fig. 10, we wish to determine the volume element $d^{3} v^{\prime}$ so that, for given $\vec{V}^{\prime}$ and $x_{g}$, the final velocity after the collision ends up in $d^{3} v$. As all angles are held constant and $\vec{V}^{\prime}$ is held fixed, the volume element $d^{3} v^{\prime}$ must be exactly similar in shape to $\mathrm{d}^{3} \mathrm{v}$, but all linear dimensions increased in the ratio $\left|\left(\vec{v}^{\prime}-\vec{v}^{\prime}\right) /\left(\vec{v}-\vec{V}^{\prime}\right)\right|$, so that

$$
d^{3} v^{\prime}=\left(\frac{\left|\vec{v}^{\prime}-\vec{v}^{\prime}\right|}{\left|\vec{v}-\vec{v}^{\prime}\right|}\right)^{3} d^{3} v
$$

Substituting this in Eq. 26.1, we obtain

$$
B=\int F\left(V^{\prime}\right) f\left(v^{\prime}\right)\left(\frac{\left|\vec{v}-\vec{V}^{\prime}\right|}{\left|\vec{v}-\vec{V}^{\prime}\right|}\right)^{3} \rho d^{2} \Omega d^{3} V^{\prime}-\int F(V) f(v) \rho d^{2} \Omega d^{3} V
$$


27. Expansion in spherical harmonics. We now substitute for $f(\vec{v})$ its expansion in spherical harmonics and for $f\left(\vec{v}^{\prime}\right)$ the corresponding series in terms of $\theta^{\prime}$. Using the addition theorem for spherical harmonics, we have

$$
f\left(\vec{v}^{\prime}\right)=\sum f^{\ell}\left[P_{\ell}(\theta) P_{\ell}\left(x_{0}\right)+2 \sum_{1}^{\ell} \frac{(\ell-m) !}{(\ell+m) !} P_{\ell}^{m}(\theta) P_{\ell}^{m}\left(x_{0}\right) \cos \vec{m}(\theta-\psi)\right]
$$

As $\rho$ has been assumed to be independent of the azimuthal angle $\psi$, the term in $\cos m(\theta-\psi)$ goes out on integrating, so that the Boltzmann integral for the coefficient of $\mathrm{P}_{\ell}(\theta)$ becomes

$$
\begin{aligned}
B^{\ell}= & \int\left(\frac{\left|\vec{v}^{\prime}-\vec{V}^{\prime}\right|}{\left|\vec{v}-\vec{V}^{\prime}\right|}\right)^{3} F\left(\vec{V}^{\prime}\right) f^{\ell}\left(v^{\prime}\right) P_{\ell}\left(x_{o}\right) \rho d^{2} \Omega d^{3} V^{\prime} \\
& -\int F(\vec{V}) f^{\ell}(v) \rho d^{2} \Omega d^{3} V
\end{aligned}
$$

So far the equations have been perfectly general, but we must now make assumptions which in practice restrict their application to electrons, or at most to light ions in a heavy gas. We shall assume that the mass ratio $\mathrm{m} / \mathrm{M}$ is zero, so that $\mathrm{v}^{\prime}=\mathrm{v}$, and that $\mathrm{F}(\overrightarrow{\mathrm{V}}) / \mathrm{n}_{\mathrm{g}}$ is a $\delta$-function. Equation 27.2 then reduces to

$$
\begin{aligned}
& \ell=0 \quad B^{O}=0 \\
& \ell \neq 0 \quad B^{\ell}=-f^{\ell}(v) n_{g} \int \rho\left(v_{0} x_{o}\right)\left[1-P_{\ell}\left(x_{o}\right)\right] d^{2} \Omega=-\nu_{c \ell} f^{\ell}
\end{aligned}
$$

The "collision frequencies" $\nu_{c \ell}$ introduced above are defined in the laboratory reference system. Introducing an arbitrary definition for $\nu_{\text {co }}$, the first three collision frequencies are

$$
\begin{aligned}
& \nu_{\mathrm{co}}=\int \mathrm{n}_{\mathrm{g}} \rho \mathrm{d}^{2} \Omega \\
& \nu_{\mathrm{c} 1}=\int \mathrm{n}_{\mathrm{g}} \rho\left(1-\cos \chi_{\mathrm{o}}\right) \mathrm{d}^{2} \Omega
\end{aligned}
$$




$$
\nu_{\mathrm{c} 2}=\int \mathrm{n}_{\mathrm{g}} \rho \frac{3}{2} \sin ^{2} x_{\mathrm{o}} \mathrm{d}^{2} \Omega
$$

The total collision frequency is $\nu_{\text {co }}$. The integral is generally improper as $\rho$ has a singularity in the forward direction. The apertures of experimental apparatus prevent observations from covering this singularity, so that the quantities tabulated as experimental "probabilities of collision" are expressed in terms of Eq. 27.5 with an incomplete integral

$$
\mathrm{P}_{\mathrm{c}}=\frac{\nu_{\mathrm{co}}^{\prime}}{\mathrm{vp_{ \textrm {o } }}}
$$

where $\mathrm{p}_{\mathrm{o}}$ is the "pressure" reduced to $0^{\circ} \mathrm{C}$.

The collision frequency for "momentum transfer, " $\nu_{c 1}$, is of such importance in transport theory that the simpler symbol $\nu_{c}$ is used for it. It is a weighted collision frequency in which the backward scattering counts double, right angle scattering has the weight one, and forward scattering is not counted. The frequency $\nu_{c 2}$ might well be called the "scattering" frequency as right-angle scattering has the weight $3 / 2$ and neither forward nor backward scattering is counted.

28. Recoil. The conclusion that $\mathrm{B}^{\circ}=0$ means that collisions do not change the energy distribution, and it follows from the assumption that the molecules were elastic and infinitely heavy. In fact, it may take many thousand collisions to change the energy of an electron appreciably whereas exactly one "collision" defined by the frequency $\nu_{\mathrm{c} 1}$ will cancel its momentum. Nevertheless, many thousand collisions do occur and we must introduce the higher-order terms into Eq. 27.2 for $\ell=0$.

We first relax the restriction to infinitely heavy molecules so as to allow the molecule to recoil under electronic impact $\left(v^{\prime}>v\right)$ but continue to neglect thermal motion $\left(V^{\prime}=0\right)$. Then $c=v^{\prime}$ and

$$
v^{\prime}{ }^{2}-v^{2}=v^{\prime} \frac{2 M m}{(M+m)^{2}}\left(1-\cos x_{g}\right)=\mu v^{\prime 2}
$$

Let us expand in terms of $\mu$ which is assumed to be small

$$
v^{\prime}{ }^{3} \rho\left(\vec{v}^{\prime}\right) f\left(\vec{v}^{\prime}\right)=v^{3} \rho f(\vec{v})+\mu v^{2} \frac{d\left(v^{3} \rho f\right)}{d\left(v^{2}\right)}
$$

Integrating the expression 27.2 for $B^{0}$ with respect to $d^{3} V^{\prime}$ for $T_{g}=0$ gives 


$$
\left.\begin{array}{rl}
B^{O} & =\int\left[\frac{v^{\prime}}{v^{3}} n_{g} \rho\left(v^{\prime}\right) f^{O}\left(v^{\prime}\right)-n_{g} \rho f^{o}(v)\right] d^{2} \Omega \\
& =\int \frac{\mu}{v} n_{g} \frac{d v^{3} \rho f^{O}}{d\left(v^{2}\right)} d^{2} \Omega=\frac{2 M m}{(M+m)^{2}} \frac{1}{v d\left(v^{2}\right)}\left(v^{3} v_{g 1} f^{o}\right)
\end{array}\right\}
$$

where $\nu_{\mathrm{g} 1}$ is the same as $\nu_{\mathrm{c} 1}$ of Eq. 27.6 but in the center of mass instead of the laboratory system. The relation between these two depends on how the cross-section $\rho$ depends on $\chi_{g}$ and is related to the "persistence of velocity" factor (22). It is given in first order by

$$
\nu_{\mathrm{c}}=\frac{\mathrm{M}}{\mathrm{M}+\mathrm{m}} \nu_{\mathrm{g} 1}
$$

so that we can write Eq. 28.3 as

$$
B^{O}=\frac{2 m}{M+m} \frac{1}{v} \frac{d}{d\left(v^{2}\right)}\left(v^{3} \nu_{c} f^{o}\right)
$$

Next we take account of the Maxwell distribution of the molecules $F\left(\vec{V}^{\prime}\right)$, which was replaced by a delta function in Eq. 28.3, and this will be done in a some what qualitative way. In integrating over $d^{3} V^{\prime}$ the point $\vec{V}^{\prime}$ of Fig. 10 moves over a Maxwell distribution centered about $\mathrm{V}^{\prime}=0$. For fixed $v^{\prime}$ and scattering angle $x_{g^{\prime}}$, this means that $\vec{v}^{\prime}$ moves about $\vec{v}_{0}^{\prime}$, its value for $V^{\prime}=0$, by amounts of the order $\vec{V}^{\prime}$.

$$
\vec{v}^{\prime}=\vec{v}_{0}^{\prime}+\mathscr{O}\left(\vec{v}^{\prime}\right)
$$

and

$$
f\left(\vec{v}^{\prime}\right)=f\left(\vec{v}_{0}^{\prime}\right)+\mathscr{O}\left(v^{\prime}{ }^{2}\right) \frac{\partial f}{\partial\left(v^{\prime}{ }^{2}\right)}
$$

This correction to $f(\vec{v})$ must enter Eq. 28.5 so that it becomes

$$
\mathrm{B}^{\mathrm{O}}=\frac{2 \mathrm{~m}}{\mathrm{M}+\mathrm{m}} \frac{1}{\mathrm{v}} \frac{\mathrm{d}}{\mathrm{d}\left(\mathrm{v}^{2}\right)}\left[\mathrm{v}^{3} \nu_{\mathrm{c}}\left(\mathrm{f}^{\mathrm{o}}+\mathscr{O}\left(\mathrm{v}^{\prime}{ }^{2}\right) \frac{\mathrm{df^{ \circ }}}{\mathrm{d}\left(\mathrm{v}^{2}\right)}\right)\right]
$$

But we know that if the electrons have a Maxwell distribution at the same temperature as that of the gas, collisions will not change this distribution and, the refore, Eq. 28.8 must give zero if $\mathrm{f}$ is a Maxwell distribution. This determines the factor $\mathscr{O}\left(\mathrm{V}^{\prime}{ }^{2}\right)$ to be equal to $2 \mathrm{k} \mathrm{T}_{\mathrm{g}} / \mathrm{m}$, and we have 


$$
\mathrm{B}^{\mathrm{O}}=\frac{2 \mathrm{~m}}{\mathrm{M}+\mathrm{m}} \frac{1}{\mathrm{v}} \frac{\mathrm{d}}{\mathrm{d}\left(\mathrm{v}^{2}\right)}\left[\mathrm{v}^{3} \nu_{\mathrm{c}}\left(\mathrm{f}^{\mathrm{O}}+\frac{2 \mathrm{kT} \mathrm{g}}{\mathrm{m}} \frac{\mathrm{df^{ \circ }}}{\mathrm{d}\left(\mathrm{v}^{2}\right)}\right)\right]
$$

for the rate of change of the energy distribution of electrons of mass $m$ owing to elastic collisions with molecules of finite mass $M$ having a temperature $\mathrm{T}_{\mathrm{g}}$.

29. Inelastic collisions. If the gas is molecular, the electrons colliding with the molecules can excite rotational and vibrational states in them. As the spacings of the rotational and vibrational energy levels are generally small compared with the mean energy of the electrons, and therefore the losses are in small amounts, of the same order as the recoil losses, these losses can be treated in the same way as the recoil losses and incorporated in Eq. 28.9. However, the excitation probabilities of rotation and vibration are not well known either theoretically (23) or experimentally; therefore they can only be taken into account by introducing an arbitrary factor $\lambda$ in place of the mass ratios $\mathrm{m} /(\mathrm{M}+\mathrm{m})$ where $2 \mathrm{~m} /(\mathrm{M}+\mathrm{m}) \leqq \lambda \leqq 1$. The two parameters $\nu_{\mathrm{c} 1}$ and $\lambda$ were introduced in the Langevin equation in Sec. 11.

In the excitation of an electronic level, of excitation potential $\mathrm{V}_{\mathrm{x}}$, the electron loses the excitation energy $\mathrm{eV}_{\mathrm{x}}$ and remains with very little energy left, generally of the order of one electron volt.

$$
\frac{1}{2} \mathrm{~m} \mathrm{v}^{2}=\frac{1}{2} \mathrm{mv}^{\prime 2}-\mathrm{eV}_{\mathrm{x}}
$$

Introducing this in the collision integral gives

$$
\int\left(\frac{v^{\prime}}{v}\right)^{3} \sum_{x} \rho_{x} f\left(v^{\prime}\right) d^{2} \Omega=\phi(v)=\xi(v) f(v)
$$

where $\phi(v)$ is the energy distribution of the electrons after the collision. It is convenient to express this function as a product $\xi(v) f(v)$, which defines the function $\xi(v)$. Because the excitation probabilities are large immediately above the excitation potential and also because the distribution function is a rapidly decreasing function of $v^{\prime}$, the functions $\phi$ or $\xi$ are both appreciable only for small velocities corresponding to the energies of the order of one electron volt. The function $\xi(v)$ corresponds to the excitation of all excitation levels, including ionization, and is therefore a sum of partial $\xi^{\prime} s$ and has no simple relation to the velocity $v^{\prime}$ of the electron before the collision. For our purpose, it will generally be sufficient to assume that the function $\xi$ is a delta-function because, as 
noted in Eq. 17.8, it is the square of the remaining energy that enters the final formulae.

If attachment or recombination is occurring this can also be included in $\xi(v)$. Introducing $\xi$ and also the total excitation frequency $\nu_{\mathrm{x}}(\mathrm{v})$ gives the inelastic term

$$
B^{O}=\left[\xi(v)-\nu_{x}(v)\right] f^{o}
$$

The integral of $\xi(v)$ is related to the inelastic, ionization, and attachment frequencies, $\nu_{\mathrm{x}}, \nu_{\mathrm{i}}$, and $\nu_{\mathrm{a}}$ because every excitation produces one slow electron, or two if it is an ionization, and attachment removes one. Therefore we have

$$
\int \xi \mathrm{f}^{\mathrm{o}} 4 \pi \mathrm{v}^{2} \mathrm{dv}=\int\left(\nu_{\mathrm{x}}+\nu_{\mathrm{i}}-\nu_{\mathrm{a}}\right) \mathrm{f}^{\mathrm{o}} 4 \pi \mathrm{v}^{2} \mathrm{dv}=\mathrm{n}\left(\bar{\nu}_{\mathrm{x}}+\bar{\nu}_{\mathrm{i}}-\bar{\nu}_{\mathrm{a}}\right)
$$

The functions $\xi$ and $\nu_{\mathrm{x}}$ generally do not overlap, $\xi$ being appreciable only for very low energies, $\nu_{\mathrm{x}}$ only for high energies, and there is a wide gap between the se two functions.

\section{c. The Drift Motion}

30. Diffusion. The results of Secs. a and b must now be put together to obtain equations for the component distribution functions $\mathrm{f}_{0}^{\circ}, \vec{f}_{0}^{1}$, and $\vec{f}_{1}^{1}$. From Eqs. 24.13 and 27.4, we have, for the dc vector equation,

$$
\left(\nu_{c}-\vec{\omega}_{b} \times\right) \vec{f}_{o}^{1}=-v \operatorname{grad} f_{o}^{o}
$$

This equation can be solved by vector methods to give

$$
\left(\nu_{c}^{2}+\omega_{b}^{2}\right) \vec{f}_{o}^{1}-\vec{\omega}_{b} \cdot \vec{f}_{o}^{1} \vec{w}_{b}=-\left(\nu_{c}-\vec{\omega}_{b} x\right) \operatorname{grad}\left(v f_{o}^{o}\right)
$$

or in terms of a matrix

$$
\overrightarrow{\mathrm{f}}_{\mathrm{o}}^{1}=-\left\|\begin{array}{ccc}
\frac{\nu_{\mathrm{c}}}{\nu_{\mathrm{c}}^{2}+\omega_{\mathrm{b}}^{2}} & \frac{\omega_{\mathrm{b}}}{\nu_{\mathrm{c}}^{2}+\omega_{\mathrm{b}}^{2}} & 0 \\
\frac{-\omega_{\mathrm{b}}}{\nu_{\mathrm{c}}^{2}+\omega_{\mathrm{b}}^{2}} & \frac{\nu_{\mathrm{c}}}{\nu_{\mathrm{c}}^{2}+\omega_{\mathrm{b}}^{2}} & 0 \\
0 & 0 & \frac{1}{\nu_{\mathrm{c}}}
\end{array}\right\| \operatorname{grad} \mathrm{f} \mathrm{f}_{\mathrm{o}}^{\mathrm{o}}
$$

This is to be compared to the tensor in expression 14.3 of Part II. The diffusion coefficient $D$ is defined in terms of the flow $\vec{\Gamma}$ by 


$$
\vec{\Gamma}=\int \frac{4 \pi}{3} \vec{f}_{0}^{1} v^{3} d v=-\operatorname{grad}(n D)
$$

$\mathrm{D}$ is a tensor whose parallel, transverse, and perpendicular components are given by

$$
\begin{aligned}
& D_{\|}=\frac{1}{n} \int \frac{v^{2}}{3 \nu_{c}} f_{o}^{o} 4 \pi v^{2} d v=\frac{\overline{\ell_{c} v}}{3} \\
& D_{T}=\frac{1}{n} \int \frac{\nu_{c}}{\nu_{c}^{2}+\omega_{b}^{2}} f_{o}^{\circ} \frac{4 \pi}{3} v^{4} d v \\
& D_{\perp}=\frac{1}{n} \int \frac{\omega_{b}}{\nu_{c}^{2}+\omega_{b}^{2}} f_{o}^{o} \frac{4 \pi}{3} v^{4} d v
\end{aligned}
$$

The diffusion coefficient $D$ has been put inside the gradient operator in the definition 30.4 because, if the electron velocity distribution $\mathrm{f}_{0}^{\mathrm{o}}$ varies from point to point, this variation must be included in taking the gradient. On the other hand, if $\nu_{c}$ varies from point to point because of its gas pressure dependence, as distinct from its velocity dependence, or if the magnetic field varies from point to point so that $\omega_{b}$ is a variable, these variations must not be included in taking the gradient. The gradient is a partial derivative operating on the distribution function and through it on the velocity dependence of $\nu_{\mathrm{c}}$ but not on its pressure dependence.

31. Mobility. The ac vector equation 24.14 is

$$
\left(\nu_{c}+j \omega-\vec{\omega}_{b} \times\right) \vec{f}_{1}^{1}=-\vec{a} \frac{d f_{o}^{o}}{d v}
$$

which is solved, as in Sec. 12, in terms of the quantities $\ell, r, p$ defined in Eq. 12.6

$$
\overrightarrow{\mathrm{f}}_{1}^{1}=-\frac{\overrightarrow{\mathrm{a}}}{2}\left\|\begin{array}{ccc}
\ell+\mathrm{r} & j(\mathrm{r}-\ell) & 0 \\
j(\ell-\mathrm{r}) & \ell+\mathrm{r} & 0 \\
0 & 0 & 2 \mathrm{p}
\end{array}\right\| \frac{\mathrm{d \textrm {f } _ { \mathrm { o } } ^ { \mathrm { O } }}}{\mathrm{dv}}
$$

We now define the quantities $L, R$, and $P$ by 


$$
L=\frac{-1}{n} \int \frac{4 \pi}{3} \ell \frac{d f_{o}^{o}}{d v} v^{3} d v=\frac{1}{n} \int f_{0}^{\circ} \frac{d}{d v}\left[\frac{4 \pi \ell v^{3}}{3}\right] d v
$$

and similar equations for $R$ and $P$ in terms of $r$ and $p$. It is noted that if $\nu_{c}$ is independent of $\mathrm{v}$, so are $\ell, \mathrm{p}$, and $\mathrm{r}$, and $\mathrm{L}=\ell, \mathrm{P}=\mathrm{p}, \mathrm{R}=\mathrm{r}$. The mobility of an electron is defined by

$$
\mu \overrightarrow{\mathrm{E}}=\overrightarrow{\mathrm{v}}_{\mathrm{d}}=\frac{1}{\mathrm{n}} \int \frac{4 \pi}{3} \overrightarrow{\mathrm{f}}_{1}^{1} \mathrm{v}^{3} \mathrm{dv}
$$

and leads to the tensor

$$
\mu=\frac{q}{2 m}\left\|\begin{array}{ccc}
L+R & j(R-L) & 0 \\
j(L-R) & L+R & 0 \\
0 & 0 & 2 P
\end{array}\right\|
$$

which reduces to Eq. 12.7 only when the mean free time, $\tau_{c}=1 / \nu_{c}$, is constant.

Expanding the expressions for the three mobility components gives

$$
\begin{aligned}
& \mu_{\| l}=\frac{q}{n m} \int f_{0}^{\circ} \frac{d}{d v}\left[\frac{\nu_{c}-j \omega}{\nu_{c}^{2}+\omega^{2}} \frac{4 \pi v^{3}}{3}\right] d v \\
& \mu_{T}=\frac{q}{n m} \int f_{0}^{0} \frac{d}{d v}\left\{\frac{\nu_{c}\left(\nu_{c}^{2}+\omega^{2}+\omega_{b}^{2}\right)-j \omega\left(\nu_{c}^{2}+\omega^{2}-\omega_{b}^{2}\right)}{\left[\nu_{c}^{2}+\left(\omega+\omega_{b}\right)^{2}\right]\left[\nu_{c}^{2}+\left(\omega-\omega_{b}\right)^{2}\right]} \frac{4 \pi v^{3}}{3}\right\} d v \\
& \mu_{\perp}=\frac{q \omega_{b}}{n m} \int f_{0}^{0} \frac{d}{d v}\left\{\frac{\nu_{c}^{2}-\omega^{2}+\omega_{b}^{2}-2 j \omega \nu_{c}}{\left[\nu_{c}^{2}+\left(\omega+\omega_{b}\right)^{2}\right]\left[\nu_{c}^{2}+\left(\omega-\omega_{b}\right)^{2}\right]} \frac{4 \pi v^{3}}{3}\right\} d v
\end{aligned}
$$

These quantities simplify considerably in the case of a direct current $(\omega=0)$ when

$$
\begin{aligned}
& \mu_{\|}=\frac{\mathrm{q}}{\mathrm{nm}} \int \mathrm{f}_{\mathrm{o}}^{\mathrm{o}} \frac{\mathrm{d}}{\mathrm{dv}}\left(\frac{4 \pi}{3} \frac{\mathrm{v}^{3}}{\nu_{\mathrm{c}}}\right) \mathrm{dv} \\
& \mu_{\mathrm{T}}=\frac{\mathrm{q}}{\mathrm{n} \mathrm{m}} \int \mathrm{f}_{\mathrm{o}}^{\mathrm{o}} \frac{\mathrm{d}}{\mathrm{dv}}\left(\frac{\nu_{\mathrm{c}}}{\nu_{\mathrm{c}}^{2}+\omega_{\mathrm{b}}^{2}} \frac{4 \pi \mathrm{v}^{3}}{3}\right) \mathrm{dv}
\end{aligned}
$$




$$
\mu_{\perp}=\frac{q}{n m} \int f_{o}^{o} \frac{d}{d v}\left(\frac{\omega_{b}}{\nu_{c}^{2}+\omega_{b}^{2}} \frac{4 \pi v^{3}}{3}\right) d v
$$

Particular interest is attached to the ratio $D / \mu$ for a direct current. From Eqs. 30.6 and 31.10 it is seen that

$$
\begin{aligned}
& \frac{\mathrm{D}}{\mu}=\frac{\mathrm{kT}}{\mathrm{e}} \quad \text { when } \mathrm{f}_{\mathrm{o}}^{\mathrm{o}}=\mathrm{A} \mathrm{e}^{-\frac{\mathrm{mv}^{2}}{2 \mathrm{kT}}} \\
& \frac{\mathrm{D}}{\mu}=\frac{\mathrm{mv}^{2}}{3 \mathrm{e}} \quad \text { when } v_{\mathrm{c}} \text { is constant } \\
& \frac{\mathrm{D}}{\mu}=\frac{\mathrm{m}}{2 \mathrm{e}} \frac{\overline{\mathrm{v}}}{\overline{1 / v}} \text { when } \nu_{\mathrm{c}}=\frac{\mathrm{v}}{\ell_{\mathrm{c}}}>\omega_{\mathrm{b}}
\end{aligned}
$$

The first of these is the well-known Einstein relation and the second is a generalization of it to non-Maxwellian distributions. The third is a warning that the generalization is not always true. Nevertheless, Eq. 31.13 is very nearly true in all practical cases.

\section{d. The Energy Distribution}

32. Plasma balance. Combining Eq. 24.12 with Eqs. 28.9 and 29.3, we obtain the equation

$\left(\nu_{x}-\xi\right) f_{o}^{o}-\frac{m}{M+m} \frac{1}{v} \frac{d}{d v}\left[v^{3} \nu_{c}\left(f_{o}^{o}+\frac{k T g}{m v} \frac{d f_{o}^{o}}{d v}\right)\right]=-\frac{v}{3} \operatorname{div} \vec{f}_{o}^{1}-\frac{1}{6 v^{2}} \frac{d}{d v}\left(v^{2} \vec{a} \cdot \vec{f} \frac{1}{1 r}\right)$

or

$$
\left(\nu_{x}-\xi\right) f_{o}^{o}=-\frac{v}{3} d i v \vec{f}_{o}^{1}-\frac{1}{4 \pi v} \frac{d G}{d v}
$$

in which we have introduced the gain

$$
\mathrm{G}=\frac{4 \pi \mathrm{v}^{2}}{3}\left[\frac{1}{2} \overrightarrow{\mathrm{a}} \cdot \overrightarrow{\mathrm{f}}_{1 \mathrm{r}}^{1}-\frac{3 \nu_{\mathrm{c}}}{\mathrm{M}+\mathrm{m}}\left(\mathrm{mv} \mathrm{f}_{\mathrm{o}}^{\mathrm{o}}+\mathrm{k} \mathrm{T}_{\mathrm{g}} \frac{\mathrm{df}_{\mathrm{o}}^{\mathrm{o}}}{\mathrm{dv}}\right)\right]
$$

This expression combines that part of the gain which was obtained in Eq. 19.8 with an additional part which represents the energy interchanges in elastic collisions with the molecules, but does not include the electronic excitations. The recoil terms are included because they consist of small energy jumps by which the electrons gain or lose energy 
almost continuously, passing through all intermediate energy values, whereas in electronic excitations, the electron jumps discontinuously from an energy near the excitation value to an energy near zero.

Multiplying Eq. 32.2 by $4 \pi \mathrm{v}^{2} \mathrm{dv}$ and integrating from zero to infinity, the gain term disappears at both limits. The other two terms have been considered in Eqs. 29.4 and 30.4 so that the net result of the integration is

$$
\nabla^{2} \mathrm{D} \mathrm{n}+\mathrm{n}\left(\bar{\nu}_{\mathrm{i}}-\bar{\nu}_{\mathrm{a}}\right)=0
$$

In computing the Laplacian in this equation, it must be remembered when there is a magnetic field present, that the diffusion coefficient has a different value along and at right angles to the magnetic field. On the other hand, the perpendicular component $D_{\perp}$ does not enter because the two off-diagonal terms of the diffusion tensor cancel each other.

Equation 32.4 is a diffusion equation and requires a characteristic value for $\bar{\nu}_{i}$ in order to satisfy prescribed boundary conditions, which are generally that $n=0$ at the walls. If $D$ is independent of position and $\Lambda_{b}$ is the diffusion length of the cavity, as defined in Eq. 13.10, then

$$
\bar{\nu}_{i}=\bar{\nu}_{\mathrm{a}}+\mathrm{D} / \Lambda_{\mathrm{b}}^{2}
$$

33. The second-order equation. Substituting from Eq. 30.3 for $\vec{f}_{0}^{1}$ in the diffusion term in Eq. 32.2, gives

$$
-\frac{\mathrm{v}}{3} \operatorname{div} \overrightarrow{\mathrm{f}}_{\mathrm{o}}^{1}=\frac{\mathrm{v}^{2}}{3} \frac{\nu_{\mathrm{c}}}{\nu_{\mathrm{c}}^{2}+\omega_{\mathrm{b}}^{2}} \nabla^{2} \mathrm{f}_{\mathrm{o}}^{\mathrm{o}} \approx-\frac{\mathrm{v}^{2}}{3 \Lambda_{\mathrm{b}}^{2} \nu_{\mathrm{c}}} \mathrm{f}_{\mathrm{o}}^{\mathrm{o}}
$$

where the replacement of $\nabla^{2}$ by $-1 / \Lambda_{b}^{2}$ is based on the assumption that the function $\mathrm{f}_{0}^{\circ}$ is the product of a function $\mathrm{n}$ of position and a normalized function of velocity only. This may not always be valid but is generally approximately so.

If we introduce the value Eq. 31.2 for $\vec{f}_{1}^{1}$ into the power input term, $\frac{1}{2} \vec{a} \cdot \vec{f}_{1}$, and make use of the drift energy expression 12.9 , it is seen that

$$
\frac{1}{2} \vec{a} \cdot \vec{f}_{1 r}^{1}=-\frac{a^{2}}{4}(\ell+r){ }_{r} \frac{d f_{o}^{o}}{d v}=-\frac{\nu_{c}}{m} 2 u_{d} \frac{d f_{o}^{o}}{d v}=-2 v v_{c} u_{d} \frac{d f_{o}^{o}}{d u}
$$

Let us define the energy gain per collision $u_{c}$ by 


$$
\begin{aligned}
& u_{c}=2 u_{d}+\frac{3 m k T_{g}}{M+m} \\
& u_{d}=\frac{e^{2} E_{p}^{2}}{8 m}\left[\frac{1}{\nu_{c}^{2}+\left(\omega-\omega_{b}\right)^{2}}+\frac{1}{\nu_{c}^{2}+\left(\omega+\omega_{b}\right)^{2}}\right]
\end{aligned}
$$

where $\omega_{b}$ is that component of the vector that is perpendicular to $E_{p}$, and in the case of a dc field the 8 is to be replaced by 4 . Introducing all this in Eqs. 32.2 and 32.3, we obtain the second-order differential equation for $f_{o}^{o}$

$$
\left.\begin{array}{l}
\left(\nu_{\mathrm{x}}-\xi+\frac{\mathrm{v}^{2}}{3 \Lambda_{\mathrm{b}}^{2} \nu_{c}}\right) \mathrm{f}_{\mathrm{o}}^{\mathrm{o}}+\frac{1}{4 \pi \mathrm{v}^{2}} \frac{\mathrm{dG}}{\mathrm{dv}}=0 \\
G=-\frac{4 \pi}{3} \mathrm{v}^{3} \nu_{\mathrm{c}}\left(\mathrm{u}_{\mathrm{c}} \frac{\mathrm{df}_{\mathrm{o}}^{\mathrm{o}}}{\mathrm{du}}+\frac{3 \mathrm{~m}}{\mathrm{M}+\mathrm{m}} \mathrm{f}_{\mathrm{o}}^{\mathrm{o}}\right)
\end{array}\right\}
$$

When $\nu_{c}$ is a constant, this equation can be solved in terms of confluent hypergeometric functions (24), but in general the solution is more difficult. In any case it is necessary to divide the solution at $\mathrm{eV}_{\mathrm{x}}$ because $\nu_{\mathrm{x}}$ is discontinuous there.

An approximation valid for high energies can be obtained by setting

$$
f_{0}^{o}=A e^{-s}
$$

and discarding all but the largest terms of Eq. 33.4 which, in the excitation range, are the terms in $\nu_{\mathrm{x}}$ and $\mathrm{u}_{\mathrm{c}}$. This leads to a Riccati equation for $\mathrm{s}^{\prime}$ :

$$
\nu_{\mathrm{x}}=\frac{2}{3} \nu_{\mathrm{c}} \mathrm{u}_{\mathrm{c}} \mathrm{u}\left(\mathrm{s}^{\prime 2}-\mathrm{s}^{\prime \prime}\right)
$$

Numerical substitutions show that for energies near the ionization potentials $\mathbf{s}^{2}>\mathbf{s}^{\prime \prime}$ so that one has, approximately,

$$
\mathrm{s}=\int \sqrt{\frac{\nu_{\mathrm{x}}}{\nu_{\mathrm{c}}} \frac{\frac{3}{2}}{\mathrm{u}_{\mathrm{c}} \mathrm{u}}} \mathrm{du} \approx \frac{\mathrm{p}}{\mathrm{E}}
$$

From this one calculates the number of inelastic collisions per ionization 


$$
N_{x}=\frac{\int_{v_{x}}^{\infty} \nu_{x} f_{o}^{o} 4 \pi v^{2} d v}{\int_{v_{i}}^{\infty} \nu_{i} f_{o}^{o} 4 \pi v^{2} d v} \approx A e^{B p / E}
$$

The calculation of the "constants" A and B requires a knowledge of the velocity dependences of $\nu_{c}$ and $\nu_{\mathrm{x}}$. They have been calculated for helium, hydrogen, and neon, and the reader is referred to the original papers for the details (25).

Note that the "average electron" approximation (Eq. 17.13) corresponds to the smaller term, s", in the Riccati equation.

34. The gain equation. Except for very large E/p, most free electrons have energies between one volt and the lowest excitation potential, and in this range $\nu_{\mathrm{x}}$ is zero and $\xi$ is small. Equation 33.4 can be multiplied by $4 \pi \mathrm{v}^{2} \mathrm{dv}$ and integrated from a velocity $\mathrm{v}$ in this range to infinity. Introducing the "diffusion frequency", we obtain

$$
\begin{aligned}
& n \nu_{d}(v)=\int_{v}^{\infty} \frac{4 \pi v^{4}}{3 \Lambda^{2} \nu_{c}} f_{o}^{o} d v=\operatorname{div} \vec{\Gamma}(u) \\
& n\left(\bar{\nu}_{x}+\nu_{d}(v)\right)=G=-\frac{4 \pi}{3} v^{3} \nu_{c}\left(u_{c} \frac{d f_{o}^{o}}{d u}+\frac{3 m}{M+m} f_{o}^{o}\right)
\end{aligned}
$$

This is the equation which Smidt (26) and, more recently, Hartman (27) have used. It is in the form of a conservation equation for electrons of speed above $v$, as indicated

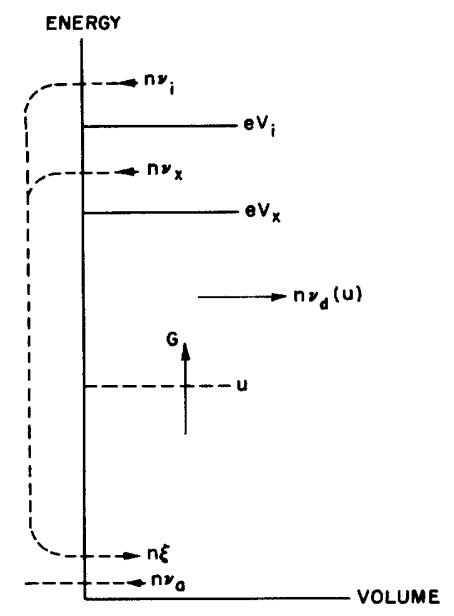

Fig. 11. Diagram to illustrate the terms in the gain equation. schematically in Fig. 11. The losses by inelastic collisions $\mathrm{n} \bar{\nu}_{\mathrm{x}}$ and diffusion $\mathrm{n} \nu_{\mathrm{d}}$ are balanced against the gain $G$ by acceleration from lower energies.

The gain function $G$ has the form shown in Fig. 12. It is constant over the range in question except for a slight drop caused by diffusion. The "diffusion frequency" $\bar{\nu}_{d}(v)$, as defined, varies from $\bar{\nu}_{i}-\bar{\nu}_{a}$ at zero, to near zero at $u_{x}$ and, knowing these limits, it can be replaced by an approximation, or when the inelastic 


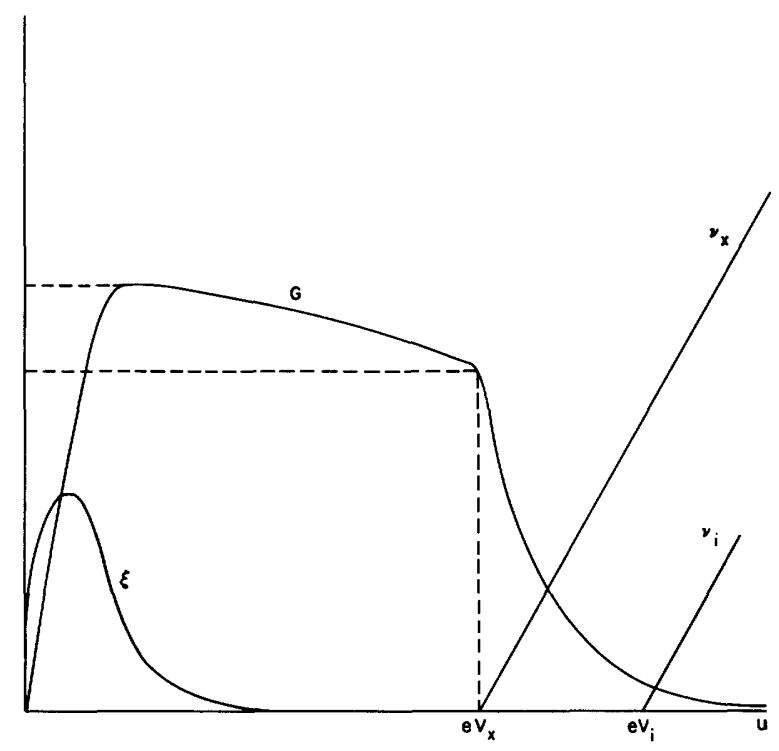

Fig. 12. The gain as a function of the energy.

collision number $\mathrm{N}_{\mathrm{x}}=\bar{\nu}_{\mathrm{x}} / \bar{\nu}_{\mathrm{i}}$ is large, as is the case except in Penning mixtures (mixtures of a rare gas with an impurity in which the rare gas metastable can ionize the impurity), it can be neglected entirely. We shall neglect it in the following. The gain equation then has a simple solution

$$
f_{o}^{o}=\frac{n \bar{\nu}_{x}}{4 \pi} \frac{M+m}{m} e^{-w} \int_{w}^{w} \frac{e^{w} d w}{v^{3} \nu_{c}}
$$

where

$$
w=\int_{0}^{u} \frac{3 m}{M+m} \frac{d u}{u_{c}}
$$

and $w_{x}$ is the value of $w$ at $u_{x}$ which is slightly above $e V_{x}$. The overshoot $u_{x}-e V_{x}$ is found by joining Eqs. 34.3 and 33.5 at $\mathrm{eV}_{\mathrm{x}}$

$$
-\frac{f_{o}^{o^{\prime}}}{f_{o}^{o}}=\frac{3 m}{M+m} \frac{1}{u_{c}}+\frac{\frac{e^{w}}{v^{3} \nu_{c} u_{c}}}{\int_{e V_{x}}^{u_{x}} \frac{e^{w} d u}{v^{3} \nu_{c} u_{c}}} \approx \frac{3 m}{M+m} \frac{1}{u_{c}}+\frac{1}{u_{x}-e V_{x}}=s_{x}^{\prime}
$$


The evaluation of $s_{x}^{\prime}$ from Eq. 33.6 is difficult, but the approximation of Eq. 17.12 turns out to be quite good.

35. The distribution function. In Sec. 16 it was seen that the average electron, in the absence of inelastic collisions, reached an equilibrium energy

$$
u_{e}=\frac{2 u_{d}}{\lambda}=\frac{M+m}{2 m} u_{c}
$$

The number $w_{x}$ is, therefore, approximately $\frac{3 u_{x}}{2 u_{e}}$. It should be less than $\frac{3}{2}$ if the equilibrium energy is to be above the excitation potential but in practice it ranges anywhere up to 2 even in discharges with active ionization. As examples, $w_{x}=\left(5 p / E_{e f}\right)^{2}$ in helium and $\mathrm{w}_{\mathrm{x}}=\left(12 \mathrm{p} / \mathrm{E}_{\mathrm{ef}}\right)^{2}$ in hydrogen, where $\mathrm{p}$ is in $\mathrm{mm}$ and $\mathrm{E}$ in volts $/ \mathrm{cm}$.

The distribution function Eq. 34.3 has two limiting forms according to the value of $\mathrm{w}_{\mathrm{x}}$. For $\mathrm{w}_{\mathrm{x}} \gg 1$, the integral is dominated by its upper limit and is practically constant. Then

$$
f_{0}^{o}=A e^{-w}
$$

This is the distribution function introduced by Druyvesteyn (28) and extended by Davydoff and Margenau (29). It is applicable when excitation losses are negligible in comparison with recoil.

At the opposite limit $w_{x} \ll 1$, or when recoil losses are negligible,

$$
f_{0}^{0}=\frac{3 n \bar{\nu}_{x}}{4 \pi} \int_{v}^{v_{x}} \frac{m d v}{v^{2} \nu_{c} u_{c}}-\frac{3 m n \bar{\nu}_{x}}{4 \pi \nu_{c} u_{c}}\left(\frac{1}{v}-\frac{1}{v_{x}}\right)
$$

where the integral has been performed on the assumption that $\nu_{c}$ is a constant. This limit is surprisingly close to the actual distribution in all medium pressure discharges in which neither diffusion, nor recoil, nor electron interaction, have taken control. It is nearly always much better than Eq. 35.2.

The exponential integral distribution Eq. 34.3 is not valid near the origin, where it has a singularity, but as all necessary integrals converge at the origin, and even contribute little from this region, the function can be used down to $v=0$. The distribution Eq. 34.3 is also invalid above $\mathrm{eV}_{\mathrm{x}}$ where the expansion Eq. 33.5 should be used, but there are relatively few electrons in the range of inelastic collisions, and even these are largely included by extending the distribution Eq. 34.5 up to $u_{x}$ where it vanishes. 
The functions

$$
\mathscr{D}_{k}=w_{x}^{-k-1} \int_{0}^{w} e^{-w} w^{k+1 / 2} d w \int_{w}^{w} e^{t} t^{-3 / 2} d t
$$

supply all necessary integrals when $\nu_{c}$ is constant, which we shall now assume. They satisfy the recursion relation

$$
\mathrm{w}_{\mathrm{x}} \mathscr{D}_{\mathrm{k}}=\left(\mathrm{k}+\frac{1}{2}\right) \mathscr{D}_{\mathrm{k}-1}-\frac{1}{\mathrm{k}}
$$

and can be computed from the series

$$
\mathscr{D}_{0}=4 \sum_{0}^{\infty} \frac{\mathrm{k} !}{(2 \mathrm{k}+3) !}\left(4 \mathrm{w}_{\mathrm{x}}\right)^{\mathrm{k}}
$$

Normalizing the distribution function yields the excitation frequency $\bar{\nu}_{\mathbf{x}}$ in terms of $\mathrm{E} / \mathrm{p}$

$$
\frac{\nu_{\mathrm{c}}}{\bar{v}_{\mathrm{x}}}=\frac{\mathrm{M}+\mathrm{m}}{2 \mathrm{~m}} \mathrm{w}_{\mathrm{x}} \mathscr{D}_{\mathrm{o}}=\frac{\mathrm{u}_{\mathrm{x}}}{\mathrm{u}_{\mathrm{o}}}\left(1+\frac{\mathrm{w}_{\mathrm{x}}}{5}+\frac{4 \mathrm{w}_{\mathrm{x}}^{2}}{105} \ldots\right)
$$

The first term, which derives from the distribution Eq. 35.3, equates the power input $\nu_{c} u_{c}$ from the field to the power loss $\bar{\nu}_{x} u_{x}$ to excitation, and the series adds the power loss to recoil.

The ionization frequency follows from this by applying Eq. 33.8:

$$
\bar{\nu}_{\mathrm{i}}=\bar{\nu}_{\mathrm{x}} / \mathrm{N}_{\mathrm{x}}
$$

The mean energy $\bar{u}$ is

$$
\bar{u}=u_{x} \frac{\mathscr{D}_{1}}{\mathscr{D}_{0}}=\frac{3}{10} u_{x} \frac{1+4 w_{x} / 21+2 w_{x}^{2} / 63 \ldots}{1+w_{x} / 5+4 w_{x}^{2} / 105 \ldots}
$$

It is generally quite reasonable to assume that the mean energy of the electrons in a plasma is $\frac{3}{10}$ of the lowest excitation potential, $\mathrm{u}_{\mathrm{x}}$ being slightly larger than $\mathrm{eV}_{\mathrm{x}}$ and the fraction being slightly less than one.

The diffusion constant, with no space charge or magnetic field is 


$$
\mathrm{D}_{-}=\frac{2}{3} \frac{\overline{\mathrm{u}}}{\mathrm{m} \nu_{\mathrm{c}}}=\frac{2}{3} \frac{\mathrm{u}_{\mathrm{x}}}{\mathrm{m} \nu_{\mathrm{c}}} \frac{\mathscr{D}_{1}}{\mathscr{D}_{\mathrm{o}}}
$$

Introducing this diffusion constant and the ionization frequency in the plasma balance equation, with no attachment, yields the breakdown condition

$$
\mathrm{mu}_{\mathrm{c}}=\left(\frac{\mathrm{u}_{\mathrm{x}}}{\Lambda \nu_{\mathrm{c}}}\right)^{2} \mathscr{D}_{1} \mathrm{~N}_{\mathrm{x}}
$$

and, in the absence of a magnetic field,

$$
\frac{1}{2}\left(\mathrm{E}_{\mathrm{p}} \Lambda\right)^{2} \frac{\nu_{\mathrm{c}}^{2}}{\nu_{\mathrm{c}}^{2}+\omega^{2}}=\frac{\mathrm{N}_{\mathrm{x}}}{5}\left(\frac{\mathrm{u}_{\mathrm{x}}}{\mathrm{e}}\right)^{2}\left(1+\frac{4 \mathrm{w}_{\mathrm{x}}}{21}+\frac{2 \mathrm{w}_{\mathrm{x}}^{2}}{63} \ldots\right)
$$

$\left(\mathrm{E}_{\mathrm{p}} \Lambda\right)$ is the voltage "applied" to the plasma (the $\frac{1}{2}$ reduces it to root-mean-square value); $\nu_{\mathrm{c}}^{2} /\left(\nu_{\mathrm{c}}^{2}+\omega^{2}\right)$ is a power factor because of the phase angle between field and current. The result is the "effective applied voltage" squared. The first term on the right comes from the distribution Eq. 35.3 and is quite close to the expression 17.8 found for the average electron. This shows that average electron calculations are reasonably good when $\mathrm{w}_{\mathrm{x}}<1$ or when the "equilibrium" electron energy $u_{e}>1.5 \mathrm{eV}_{\mathrm{x}}$, that is, for high $\mathrm{E} / \mathrm{p}$. The power series in Eq. 35.12 represents recoil losses.

In general, the collision frequency $\nu_{c}$ is a complicated function of the velocity. It is convenient mathematically to approximate it by a power law

$$
\nu_{c}=\mathrm{c} \mathrm{v}{ }^{\mathrm{h}-1}
$$

although there is no physical basis for this law and, in fact, it is generally not a good approximation. However, it does include the important constant mean-free-time $(h=1)$ and constant mean-free-path $(h=2)$ laws and, in general, it illustrates the effects of rising or falling cross sections. This law can be introduced in the integral function 34.3 , but the series corresponding to the functions $\mathscr{D}_{\mathrm{k}}$ have not been worked out and, since there is some labor involved, it may not be worth while for this rather crude approximation. However, there are three limiting forms of distribution: the Druyvesteyn (Eq. 35.2), the "cutoff" (Eq. 35.3), and the Maxwell distribution, which stand, so to speak, at the corners of a triangle, all other distribution functions falling between them. They correspond to low $\mathrm{E} / \mathrm{p}, \mathrm{high} \mathrm{E} / \mathrm{p}$, and high degree of ionization (see Sec. 54). It seems worth while to tabulate the results of 
Each distribution function has a characteristic energy $u_{f}$, and corresponding velocity $\mathrm{v}_{\mathrm{f}}$, at which the collision frequency is $\nu_{\mathrm{cf}}$. The mean velocity $\overline{\mathrm{v}}$, mean energy $\overline{\mathrm{u}}$, mobility $\mu$, and diffusion constant $\mathrm{D}$ are given in terms of these energies in the following table:

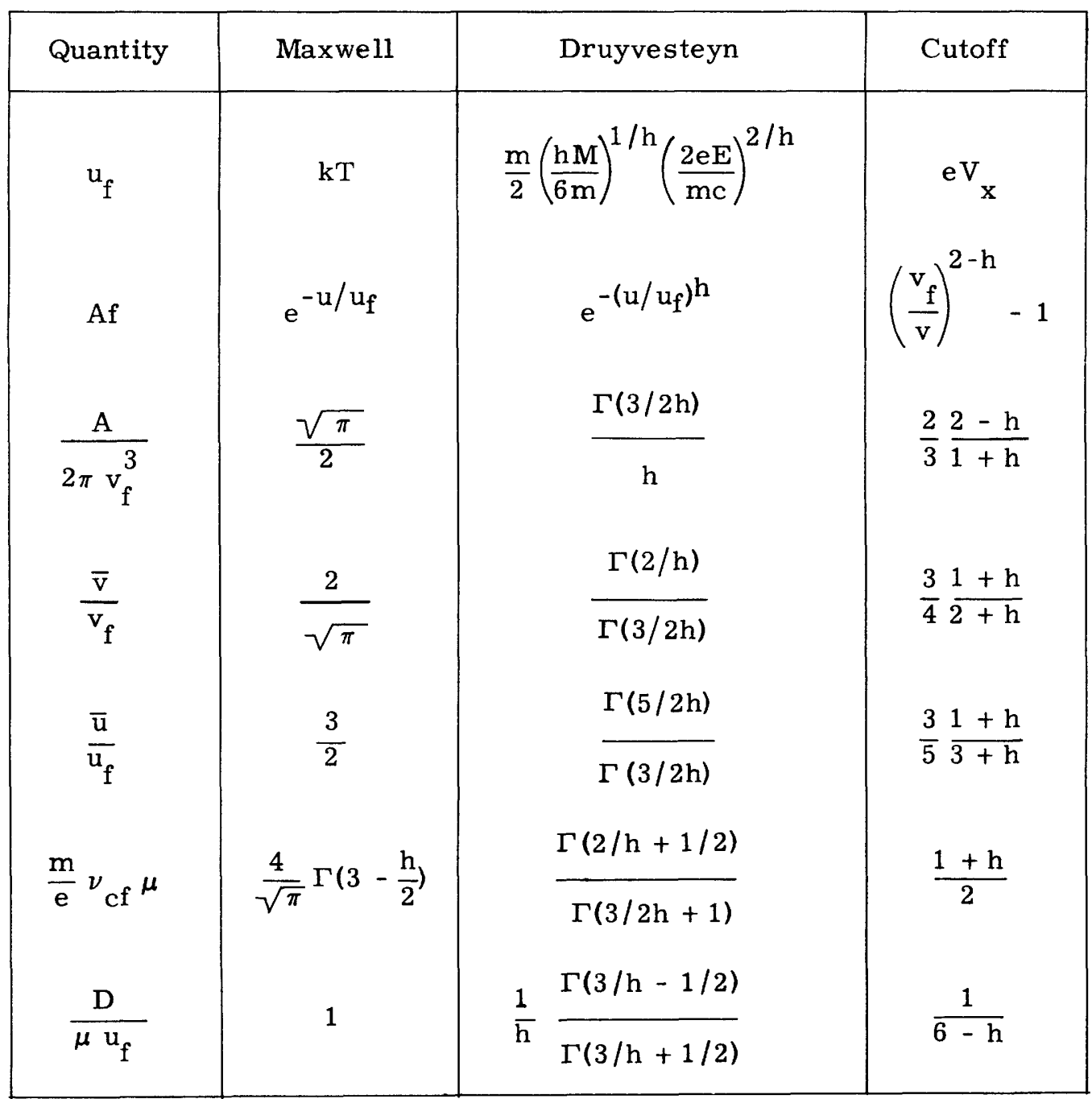


For the Druyvesteyn distribution, it is seen that the normalization integral diverges for $h=0$, and the diffusion integral for $h=6$. All powers between these limits are acceptable. The ratios of gamma functions are evaluated for a few powers in the following table:

\begin{tabular}{|c|c|c|c|c|}
\hline $\mathrm{h}$ & $\frac{\overline{\mathrm{v}}}{\mathrm{v}_{\mathrm{f}}}$ & $\frac{\overline{\mathrm{u}}}{\mathrm{u}_{\mathrm{f}}}$ & $\frac{\mathrm{m}}{\mathrm{e}} \nu_{\mathrm{cf}} \mu$ & $\frac{\mathrm{D}}{\mu \mathrm{u}_{\mathrm{f}}}$ \\
\hline 0 & $\infty$ & $\infty$ & $\infty$ & $\infty$ \\
$1 / 2$ & 3 & 12 & 3.87 & 9 \\
1 & 1.128 & 1.5 & 1 & 1 \\
$3 / 2$ & .8931 & .9028 & .9407 & .627 \\
2 & .817 & .740 & .965 & .565 \\
3 & .765 & .636 & 1.049 & .636 \\
\hline
\end{tabular}

\section{e. Direct Current}

36. The first Townsend coefficient. The energy equations of the previous section can all be applied to direct current discharges by simply setting $\omega=0$ and replacing the root-mean-square field by the dc field. Only the plasma balance equation must be reinterpreted because electrons may leave the plasma by mobility as well as by diffusion. For a long column, the plasma balance equation remains as it is in Eq. 32.3, except that the diffusion length is computed for the two lateral directions only.

For a short column, such as is used in a Townsend discharge, the lateral loss is generally negligible but we must consider the longitudinal diffusion. We shall consider the case of no magnetic field, space charge, or attachment, and shall define the first Townsend coefficient $\alpha$ by assuming a simple exponential increase in the direction of the field

$$
f(z, v)=f(v) e^{\alpha z}
$$


The vector equation, found by combining Eqs. 30.1 and 31.1, is

$$
\left.\begin{array}{rl}
\nu_{c} \vec{f}^{1} & =-v \operatorname{grad} f^{o}-\vec{a} \frac{d f^{\circ}}{d v} \\
& =-\left(\alpha \vec{v}+\vec{a} \frac{d}{d v}\right) f^{o}
\end{array}\right\}
$$

Setting this in Eq. 32.2, we obtain

$$
\left(\nu_{\mathrm{X}}-\xi\right) \mathrm{f}^{\mathrm{O}}=\frac{\alpha \mathrm{v}}{3 v_{\mathrm{C}}}\left(\alpha \mathrm{v}+\mathrm{a} \frac{\mathrm{d}}{\mathrm{dv}}\right) \mathrm{f}^{\mathrm{O}}-\frac{1}{4 \pi \mathrm{v}^{2}} \frac{\mathrm{dG}}{\mathrm{dv}}
$$

Multiplying by $4 \pi v^{2} d v$ and integrating from zero to infinity, we get

$$
\left.\begin{array}{rl}
\mathrm{n} \bar{\nu}_{\mathrm{i} \alpha} & =-\int \frac{\alpha^{2} \mathrm{v}^{2}}{3 \nu_{\mathrm{c}}} \mathrm{f}^{\mathrm{o}} 4 \pi \mathrm{v}^{2} \mathrm{dv}-\int \frac{\alpha \mathrm{a}}{3 \nu_{\mathrm{c}}} \frac{\mathrm{df}^{\mathrm{O}}}{\mathrm{dv}} 4 \pi \mathrm{v}^{3} \mathrm{dv} \\
\bar{\nu}_{\mathrm{i} \alpha} & =\alpha \mathrm{E} \mu-\alpha^{2} \mathrm{D}=\alpha \mathrm{v}_{\mathrm{d}}
\end{array}\right\}
$$

where $v_{d}$ is the resultant drift velocity arising from both field and concentration gradient.

This gives the relation between the ionization frequency $\bar{\nu}_{i \alpha}$ and the coefficient $\alpha$ in a Townsend discharge, and it is easy to show that the diffusion term is small. If we introduce $\eta=\alpha / \mathrm{E}$, the number of ionizations per volt, then the ratio of the two terms in $\bar{\nu}_{i \alpha}$ is

$$
\frac{\alpha \mathrm{D}}{\mu \mathrm{E}}=\eta \frac{2 \overline{\mathrm{u}}}{3 \mathrm{e}} \leqq \frac{\eta \mathrm{x}}{5 \mathrm{e}}<\frac{1}{5}
$$

Except for a Penning mixture, in which $\eta u_{x} / e$ may be close to 1 , the diffusion term is always much less than one fifth of the mobility term.

However, we would like to compare $\alpha$ with $\bar{\nu}_{i}$ in a uniform discharge of the same E/p. Substituting Eq. 36.2 in the gain equation 32.3 , we obtain

$$
G=-\frac{4 \pi}{3} v^{3} \nu_{c}\left[\left(2 u_{d}+\frac{3 m}{M+m} k T_{g}\right) \frac{d f^{\circ}}{d u}+\left(\frac{3 m}{M+m}+\frac{\alpha a}{\nu_{c}^{2}}\right) f^{\circ}\right]
$$

and it is seen that the nonuniformity is equivalent to an increase in the recoil loss. From Eq. 34.4, it follows that $\mathrm{w}_{\mathrm{x}}$ is increased

$$
w_{x \alpha}=w_{x}+\eta u_{x} l e
$$

and introducing this in Eq. 35.7 , we get the ratio 


$$
\frac{\bar{\nu}_{i}}{\nu_{i \alpha}}=\frac{1+\frac{w_{x \alpha}}{5}+\frac{4 w_{x \alpha}^{2}}{105} \ldots}{1+\frac{w_{x}}{5}+\frac{4 w_{x}^{2}}{105} \ldots}=1+\frac{\eta u_{x} / e}{5+w_{x}} \ldots
$$

The nonuniformity reduces $\bar{\nu}_{i}$ in just about the same ratio as $v_{d}$ is reduced by diffusion. Unless $\eta u_{x} / e$ is unusually large, one can write

$$
\bar{\nu}_{i}=\alpha \mu \mathrm{E}
$$

and

$$
\frac{1}{\eta}=\frac{E}{\alpha}=\mathrm{N}_{\mathrm{x}} \mathrm{u}_{\mathrm{x}}\left(1+\frac{\mathrm{w}_{\mathrm{x}}}{5}+\frac{4 \mathrm{w}_{\mathrm{x}}^{2}}{105}+\ldots\right)
$$

The variation of $\eta$ with $\mathrm{E} / \mathrm{p}$ is attributable almost entirely, except for a Penning mixture, to the variation of $\mathrm{N}_{\mathrm{x}}$ which, as has been seen, varies as $\mathrm{e}^{\mathrm{Bp} / \mathrm{E}}$. At low $\mathrm{E} / \mathrm{p}$ this must be corrected by the series in Eq. 36.10 which is caused by recoil. At high $\mathrm{E} / \mathrm{p}$ the overshoot $u_{x}-e V_{x}$ causes a decrease in $\eta$. The transition between a decreasing $\mathrm{N}_{\mathrm{x}}$ and an increasing $\mathrm{u}_{\mathrm{x}}$ results in a maximum for $\eta$ which corresponds to the Paschen minimum in dc breakdown. 


\section{BOLTZMANN TRANSPORT EQUATION}

\section{a. General Theory}

37. Transport equation. In the case of ions whose mass $\mathrm{m}$ is comparable to the mass $M$ of the molecules of the gas, the expansion 19.2 in spherical harmonic requires more than two terms, and it is no longer possible to obtain as simple an equation as 33.4 for the distribution function $\mathrm{f}^{\mathrm{O}}(\mathrm{v})$; but it is possible to transform the Boltzmann equation as given by Eqs. 18.4 and 26.1 in such a way that it may be possible to calculate the average value of any quantity $X(v)$ without knowing too much about the distribution function $\mathrm{f}$.

The Boltzmann equation is

$$
\begin{aligned}
& {\left[\frac{\partial f}{\partial t}+\operatorname{div}_{r} \overrightarrow{v f}+\operatorname{div}_{v}\left(\vec{a}+\vec{w}_{b} \times \vec{v}\right) f\right] d^{3} v=} \\
& \quad=\iint F\left(V^{\prime}\right) f\left(v^{\prime}\right) \rho d^{2} \Omega d^{3} V^{\prime} d^{3} v^{\prime}-\iint F(V) f(v) \rho d^{2} \Omega d^{3} V d^{3} v
\end{aligned}
$$

Multiplying both sides of this equation by $X(v)$ and integrating over the velocities of the ions gives

$$
\begin{aligned}
& \frac{\partial}{\partial t}(n \bar{x})+\operatorname{div}_{r}(n \overrightarrow{\vec{v} X})+\int X \operatorname{div}_{v}\left(\vec{a}+\vec{w}_{b} \times \vec{v}\right) f d^{3} v= \\
& \quad=\iiint X F\left(V^{\prime}\right) f\left(v^{\prime}\right) \rho d^{2} \Omega d^{3} v^{\prime} d^{3} v^{\prime}-\iiint X F(V) f(v) \rho d^{2} \Omega d^{3} V d^{3} v
\end{aligned}
$$

where the bar indicates an average. The third term on the left can be transformed by Green's theorem, and in the first integral on the right it is allowable to interchange primed and unprimed variables because of the reversibility of elastic collisions. This leads to the transport equation 


$$
\begin{aligned}
& \frac{\partial}{\partial t}(n \bar{X})+\operatorname{div}_{r}(\overline{n \vec{v}})-n\left(\vec{a} \cdot \operatorname{grad}_{v}\right) X-n\left(\overrightarrow{\left.\vec{\omega}_{b} \times \vec{v} \cdot \operatorname{grad}_{v}\right) X}=\right. \\
& =\iiint\left(X^{\prime}-X\right) F(V) f(v) \rho d^{2} \Omega d^{3} V d^{3} v
\end{aligned}
$$

The left-hand terms of this equation determine the rate of change of $n \bar{X}$ caused by flow in phase space. The right-hand side gives the average rate of change of $\mathrm{X}$ caused by collisions. We shall use $X=1$ and $X=\vec{v}$ as illustrations of this equation.

For $\mathrm{X}=1, \overrightarrow{\mathrm{v} X}=\overrightarrow{\mathrm{v}}_{\mathrm{d}}$. On the right $\mathrm{X}^{\prime}=\mathrm{X}$ for an elastic collision, but inelastic collisions can be taken into account by letting $X^{\prime}=2$ in an ionizing collision, or $X^{\prime}=0$ for an attachment or recombination. Then the transport equation gives

$$
\frac{\partial \mathrm{n}}{\partial \mathrm{t}}+\operatorname{div}\left(\mathrm{n} \overrightarrow{\mathrm{d}}_{\mathrm{d}}\right)=\mathrm{n}\left(\bar{\nu}_{\mathrm{i}}-\bar{\nu}_{\mathrm{a}}\right)
$$

For $X=\vec{v}, \operatorname{grad} X=1$, and the transport equation becomes

$$
\frac{\partial}{\partial t}\left(n \vec{v}_{d}\right)+\frac{\operatorname{div} \stackrel{\leftrightarrow}{m}}{m}-n\left(\vec{a}+\vec{\omega}_{b} \times \vec{v}_{d}\right)=-n \bar{\nu}_{c} \vec{v}_{d}
$$

where the pressure $\overleftrightarrow{\mathrm{p}}$ is a symmetric tensor.

$$
p_{i j}=n m \overline{v_{i} v_{j}}
$$

and the right-hand side is really a definition of $\bar{\nu}_{\mathrm{c}}$.

The collision term will be discussed in the following sections, but the pressure term will be discussed immediately. For a nearly spherically symmetric distribution the tensor reduces to the usual scalar

$$
\mathrm{p}=\mathrm{nmv} \overline{\mathrm{v}^{2}} / 3=\mathrm{nkT}
$$

If the inelastic term $n\left(\bar{\nu}_{i}-\bar{\nu}_{\mathrm{a}}\right)$ is neglected, Eqs. 37.4 and 37.5 combine to give

$$
\frac{\partial \vec{v}_{d}}{\partial t}=\vec{a}+\vec{\omega}_{b} \times \vec{v}_{d}-\frac{\operatorname{grad} p}{n m}-\vec{v}_{c} \vec{v}_{d}+\vec{v}_{d} \frac{\operatorname{div}\left(n \vec{v}_{d}\right)}{n}
$$

This is to be compared with Eq. 8.1, which was obtained under simpler assumptions, and has, in addition to the collision term not considered in Sec. 8, a term quadratic in $\vec{v}_{d}$. 
This term is generally negligible if one is justified in replacing $\overleftrightarrow{\mathrm{p}}$ by a scalar.

Let us now consider a group of ions whose velocities are all clustered about $\vec{v}_{d}$. The pressure tensor $\overleftrightarrow{\mathrm{p}}$ has then a single component

$$
\mathrm{p}_{\mathrm{zz}}=n m v_{\mathrm{d}}^{2}
$$

The quadratic terms now add in such a way that they give

$$
\frac{d \vec{v}_{d}}{d t}=\frac{\partial \vec{v}_{d}}{\partial t}+\left(\vec{v}_{d} \cdot \text { grad }\right) \vec{v}_{d}=\vec{a}+\vec{\omega}_{b} \times \vec{v}_{d}-\vec{v}_{c} \vec{v}_{d}
$$

This is the same thing as the Langevin equation 12.1 for $\mathrm{v}_{\mathrm{g}}$. Beside referring to different situations ( $\vec{v}_{d}$ to small drift motions, $\vec{v}_{g}$ to small random motions), the drift and guiding center methods are similar to the Euler and Lagrange methods in hydrodynamics.

38. Recursion equations. We now simplify the transport equation 37.3 by considering the steady state with no density gradient or magnetic field. Only the term in $\vec{a}$ remains on the left. The collision integral on the right is quite intractable without approximations except in the case of a constant mean free time, treated in section $b$. The usual assumption, treated by Chapman and Cowling (20), is to consider small departures from thermal equilibrium. It turns out, as discussed in Sec. 43, that for ions small departures from thermal equilibrium fall under the mean-free-time assumption, which is treated exactly. We, therefore, make the opposite assumption, that the gas temperature is negligible compared with the random energy of the ions. The distribution function $\mathrm{F}$ then becomes $\mathrm{n}_{\mathrm{g}}$ times a $\delta$-function and the integration over $\overrightarrow{\mathrm{V}}$ is immediate. In this way, the transport equation becomes

$$
\int f(\vec{a} \cdot \operatorname{grad}) X d^{3} v=\iint\left(X-X^{\prime}\right) f n_{g} \rho d^{2} \Omega d^{3} v
$$

We shall consider a set of quantities $\mathrm{X}$ of the form

$$
\mathrm{X}=\mathrm{v}^{\mathrm{k}} \mathrm{P}_{\boldsymbol{l}}(\theta)
$$

where $\vec{a}$ is in the direction $\theta=0$, and derive recursion relations between averages $\mathrm{x}_{l}^{\mathrm{k}}$ of quantities of this form with different values of $k$ and $\ell$. 
Using the formulae for spherical harmonics 19.9 and 19.10 , we then find for $\vec{a} \cdot \operatorname{grad} X$.

$$
\int \mathrm{f}(\overrightarrow{\mathrm{a}} \cdot \mathrm{grad}) \mathrm{v}^{\mathrm{k}} \mathrm{P}_{\ell} \mathrm{d}^{3} \mathrm{v}=\mathrm{n} \mathrm{a}\left[\frac{\ell(\mathrm{k}+\boldsymbol{\ell}+1)}{2 \ell+1} \mathrm{x}_{\ell-1}^{\mathrm{k}-1}+\frac{(\ell+1)(\mathrm{k}-\ell)}{2 \ell+1} \mathrm{x}_{\ell+1}^{\mathrm{k}-1}\right]
$$

Using the rotation formula for spherical harmonics Eq. 27.1, we obtain

$$
X^{\prime}=v^{\prime k}\left[P_{\ell}(\theta) P_{\ell}\left(x_{0}\right)+2 \sum \frac{(l-m) !}{(\ell+m) !} P_{\ell}^{m}(\theta) P_{\ell}^{m}\left(x_{o}\right) \cos m(\phi-\psi)\right]
$$

and introducing this into the collision integral 38.1, the term in $\cos \mathrm{m}(\phi-\psi)$ integrates out because $\rho$ is not a function of $\phi$. The spherical harmonic $P_{\ell}(\theta)$ then factors out and the only part of this integral that depends on the scattering angle $x$ is

$$
\mathrm{I}_{\ell}^{\mathrm{k}}=\int\left[1-\left(\frac{\mathrm{v}^{\prime}}{\mathrm{v}}\right)^{\mathrm{k}} \mathrm{P}_{\ell}\left(\mathrm{x}_{\mathrm{o}}\right)\right] \mathrm{n}_{\mathrm{g}} \rho \mathrm{d}^{2} \Omega
$$

The velocity ratio is obtained from the geometry of a collision

$$
\frac{v^{\prime}}{v}=\frac{\sqrt{M^{2}+2 M m \cos x_{g}+m^{2}}}{M+m}
$$

and the laboratory scattering angle $x_{0}$ is given in terms of the center of gravity angle $x_{g}$ by

$$
\cos x_{0}=\frac{m+M \cos x_{g}}{\sqrt{M^{2}+2 M m \cos x_{g}+m^{2}}}
$$

For the particular case of an ion of the same mass as the gas, $M=m$, these formulae reduce to

$$
\frac{v^{\prime}}{v}=\cos x_{0}=\cos \frac{x_{g}}{2}
$$

and the calculations are greatly simplified. This case has been worked out in detail by Wannier (30) whose work we follow. 
The integrals $\mathrm{I}_{\ell}^{\mathrm{k}}$ depend in unknown ways on the speed $\mathrm{v}$, but we shall assume a power law, so that they can be written

$$
\mathrm{I}_{\ell}^{\mathrm{k}}=\mathrm{N}_{\ell}^{\mathrm{k}} \mathrm{qv} \mathrm{v}^{\delta}
$$

where the $\mathrm{N}_{\ell}^{\mathrm{k}}$ are pure numbers and $\mathrm{qv}{ }^{\delta}$ has the dimensions of a frequency. For a constant mean free time $\delta=0, \mathrm{q}=\nu_{\mathrm{g}}$. For a constant mean free path $\delta=1, \mathrm{q}=1 / \ell_{\mathrm{g}}$.

Introducing Eqs. 38.4 and 38.9 in the transport equation leads to the recursion relations

$$
\frac{\mathrm{a}}{\mathrm{q}}\left[\ell(\mathrm{k}+\ell+1) \mathrm{x}_{\ell-1}^{\mathrm{k}-1}+(\ell+1)(\mathrm{k}-\ell) \mathrm{x}_{\ell+1}^{\mathrm{k}-1}\right]=(2 \ell+1) \mathrm{N}_{\ell}^{\mathrm{k}} \mathrm{x}_{\ell}^{\mathrm{k}+\delta}
$$

Wannier has shown how to solve these equations in the cases $\delta=0$ and $\delta=1$.

\section{b. Constant Mean Free Time}

39. Drift velocity. Maxwell (31) pointed out that in the case of the inverse fifth power law of force, because it gives a constant mean free time, all transport quantities can be calculated without any knowledge of the distribution function $f$. It is not even necessary to know the molecular function $\mathrm{F}$ and we can therefore use the general transport equation 37.3 . Let

$$
\mathrm{X}=\overrightarrow{\mathrm{v}}
$$

Then

$$
(\vec{a} \cdot \operatorname{grad}) \vec{v}=\vec{a}
$$

and therefore

$$
n \vec{a}=\iiint\left(\vec{v}-\vec{v}^{\prime}\right) F \text { f } \rho d^{2} \Omega d^{3} v d^{3} v
$$

From the geometry of a collision, averaging over the azimuthal scattering angle $\psi$ (see Fig. 10) gives

$$
\left\langle\vec{v}-\vec{v}^{\prime}\right\rangle_{\psi}=\frac{M}{M+m}\left(1-\cos x_{g}\right)(\vec{v}-\vec{v})
$$

Integrating over the scattering angle $x_{g}$, and defining collision frequencies $\nu_{\mathrm{g}}$ in the center of gravity system in complete analogy to those, $v_{\mathrm{c}}$, in the laboratory system (Eqs. 27.5 to 27.7), one obtains 


$$
n \vec{a}=\frac{M}{M+m} \iint F f \frac{\nu^{1}}{n_{g}}(\vec{v}-\vec{V}) d^{3} V d^{3} v
$$

The linear term in $\mathrm{V}$ disappears on integrating over $\mathrm{V}$ and, thanks to the independence of $v_{\mathrm{g} 1}$ on $|\overrightarrow{\mathrm{v}}-\overrightarrow{\mathrm{V}}|$, one obtains

$$
\vec{a}=\frac{M}{M+m} \nu_{g 1} \vec{v}_{d}=\nu_{c 1} \vec{v}_{d}
$$

The expression with $\nu_{c}$ is obtained from Eq. 37.5 and this shows the relation between $\nu_{c}$ and $\nu_{g}$, the collision frequency in the laboratory and the center of gravity system. The factor $\frac{M+m}{M}$ is known as the persistence of velocity factor. If the scattering is isotropic in the center of gravity system, it is not isotropic in the laboratory system, owing to the recoil of the molecule, resulting in an average forward velocity of the scattered ion after a collision. As the frequency defined by Eq. 27.6 is the average frequency with which the forward motion is annulled by collisions, a "collision" in the center of gravity system is only a "fractional collision" in the laboratory system and the laboratory collision frequency is slightly less than the center of gravity collision frequency. Similarly, the laboratory mean free path $\ell_{c}$ is slightly longer than the center of gravity mean free path $\ell_{g}$. Substituting for $\vec{a}$, we obtain the mobility

$$
\mu=\frac{\mathrm{v}_{\mathrm{d}}}{\mathrm{E}}=\frac{\mathrm{e}}{\mathrm{m}_{\mathrm{r}} \nu_{\mathrm{g} 1}}=\frac{\mathrm{e}}{\mathrm{m} \nu_{\mathrm{c}}}
$$

where $m_{r}=M m /(M+m)$ is the reduced mass. When one is comparing situations which differ only in the masses, as when different isotopes of the same elements are used, $\nu_{\mathrm{g}}$ and not $\nu_{c}$ remains the same, and therefore the mobility changes with the reduced mass.

40. Energy. Let

$$
\mathrm{X}=\mathrm{v}^{2}
$$

whence

$$
\int f(\vec{a} \cdot \operatorname{grad}) v^{2} d^{3} v=\int f 2 \vec{a} \cdot \vec{v} d^{3} v=2 \vec{a} \cdot \vec{v}_{d}
$$

It is convenient to consider the velocity $\vec{v}$ as the sum of the velocity $\vec{v}_{g}$ of the center of gravity plus a fraction of the relative velocity $\vec{c}$

$$
\vec{v}=\vec{v}_{g}+\frac{M}{M+m} \vec{c}
$$


We then have

$$
(M+m)^{2} v^{2}=(M+m)^{2} v_{g}^{2}+2 M(M+m) \vec{v}_{g} \cdot \vec{c}+M^{2} c^{2}
$$

and, as $\overrightarrow{\mathrm{v}}_{\mathrm{g}}$ and the magnitude $\mathrm{c}$ are conserved in a collision,

$$
(M+m)\left(v^{2}-v^{\prime}\right)=2 M \vec{v}_{g} \cdot\left(\vec{c}-\vec{c}^{\prime}\right)
$$

Averaging over the azimuthal angle gives

$$
(M+m)<v^{2}-v^{\prime 2}>_{\psi}=2 M \vec{v}_{g} \cdot \vec{c}\left(1-\cos x_{g}\right)
$$

Averaging over scattering angles gives

$$
(\mathrm{M}+\mathrm{m})<\mathrm{v}^{2}-\mathrm{v}^{2}>_{\Omega}=2 \mathrm{M} \overrightarrow{\mathrm{v}}_{\mathrm{g}} \cdot \overrightarrow{\mathrm{c}}_{\mathrm{g} 1} / \mathrm{n}_{\mathrm{g}}
$$

or substituting back for $\vec{v}_{\mathrm{g}}$ and $\vec{c}$

$$
(\mathrm{M}+\mathrm{m})^{2}\left\langle\mathrm{v}^{2}-\mathrm{v}^{2}\right\rangle_{\Omega}=2 \mathrm{M}(\mathrm{m} \overrightarrow{\mathrm{v}}+\mathrm{M} \overrightarrow{\mathrm{V}}) \cdot(\overrightarrow{\mathrm{v}}-\overrightarrow{\mathrm{V}}) \nu_{\mathrm{g} 1} / \mathrm{n}_{\mathrm{g}}
$$

If we substitute in the transport equation, the terms in $\vec{v} \cdot \vec{V}$ drop out on integrating over the distribution function $\mathrm{F}$ for the molecules. Integrating over the ion velocities gives

$$
(M+m)^{2} \vec{a} \cdot \vec{v}_{d}=M\left(m \overline{v^{2}}-M \overline{v^{2}}\right) \nu_{g 1}
$$

Substituting for $\vec{a}$ in terms of $\vec{v}_{d}$ from Eq. 39.6 gives

$$
(M+m) v_{d}^{2}=m \bar{v}^{2}-M \overline{v^{2}}
$$

or for the random energy

$$
m \overline{v_{r}^{2}}=M\left(\overline{v^{2}}+v_{d}^{2}\right)
$$

At first, it appears surprising that the drift velocity in this equation should be associated with the mass of the molecule rather than with that of the ion. However, if we consider ourselves in the system moving with the drift velocity of the ions, Eq. 40.11 says that the mean energy of the ions is equal to the mean energy of the molecules, 
composed of their random energy and drift energy relative to the ions. This is, therefore, an application of the equipartition theorem and, viewed this way, exactly what one might expect.

The above calculations can be repeated with a component energy

$$
\mathrm{x}=\mathrm{v}_{\mathrm{x}}^{2} \text { and } \overrightarrow{\mathrm{a}} \cdot \operatorname{grad} \mathrm{v}_{\mathrm{x}}^{2}=0
$$

The collision integral leads, after somewhat more difficult manipulations, to the relation

$$
6 \nu_{\mathrm{g} 1}\left(\mathrm{~m} \mathrm{v}_{\mathrm{x}}^{2}-\mathrm{M} \mathrm{v}_{\mathrm{x}}^{2}\right)=\nu_{\mathrm{g} 2} \mathrm{M}\left(\mathrm{v}_{\mathrm{z}}^{2}-\mathrm{v}_{\mathrm{x}}^{2}\right)
$$

where ${ }_{\mathrm{g} 2}$ is the collision frequency that emphasizes right-angle scattering Eq. 27.7. Combining this with Eq. 40.11 gives the ellipticity in the distribution of the random energy

$$
\xi \equiv \frac{\overline{\mathrm{v}}_{\mathrm{rz}}^{2}-\overline{\mathrm{v}_{\mathrm{x}}^{2}}}{\overline{\mathrm{v}_{\mathrm{x}}^{2}}}=3 \frac{2 \nu_{\mathrm{g} 1}-\nu_{\mathrm{g} 2}}{\nu_{\mathrm{g} 2}} \frac{\overline{\mathrm{m} \mathrm{v}_{\mathrm{x}}^{2}}-\mathrm{M} \overline{\mathrm{v}_{\mathrm{x}}^{2}}}{(\mathrm{~m}+\mathrm{M}) \overline{\mathrm{v}_{\mathrm{x}}^{2}}}
$$

For low drift velocities, $\mathrm{m} \overline{\mathrm{v}_{\mathrm{x}}}=\mathrm{M} \overline{\mathrm{v}_{\mathrm{x}}^{2}}$, and, in general, for light ions such as electrons, the ellipticity is small and the distribution is almost spherical about the drift velocity $\vec{v}_{d}$. However, for heavy ions, $m>M$, the distribution about $\vec{v}_{d}$ is such that the random velocity in the direction of the drift is greater than the random velocity at right angles to the drift. If the scattering frequency $\nu_{\mathrm{g} 2}$ is small, there may be very little random velocity at right angles to the direction of drift, or if $\nu_{\mathrm{g} 2}=2 \nu_{\mathrm{g} 1}$, which is unlikely, the ellipticity vanishes in all cases.

Similar calculations can be carried through for all higher moments of the distribution function and therefore the distribution function $\mathrm{f}$ can itself be determined. However, it is not necessary to do so in order to get the moments, and, as the latter are the desired quantities, there is no purpose in calculating the distribution.

\section{c. Constant Mean Free Path}

41. Heavy ions. It is seen from Eq. 40.11 that the random velocity of a heavy ion in a light gas $(m>M)$ is small relative to the drift velocity, so that a heavy ion moves like a particle falling in a viscous medium, its distribution function resembling a deltafunction. As a better approximation, we can give the distribution function the form of a gaussian function 


$$
\mathrm{f}=\mathrm{A} \exp \left(-\frac{\mathrm{m}}{2 \mathrm{M}} \frac{3+\mathscr{E}}{\mathrm{v}^{2}+\mathrm{v}_{\mathrm{d}}^{2}}\left[\mathrm{v}_{\mathrm{x}}^{2}+\mathrm{v}_{\mathrm{y}}^{2}+\frac{\left(\mathrm{v}_{\mathrm{z}}-\mathrm{v}_{\mathrm{d}}\right)^{2}}{1+\mathscr{E}}\right]\right)
$$

Since the random velocity is small compared with the drift velocity, the actual velocity is nearly a constant and, therefore, it does not matter how the collision frequency varies with velocity. The distribution Eq. 41.1 is good in general for a heavy ion, and so is the equation 39.6 for the drift velocity, provided that $\nu_{\mathrm{g}}$ is evaluated for the drift velocity.

Applying this to the case of a constant mean free path

$$
\ell_{\mathrm{g}}=\frac{\mathrm{v}}{\nu_{\mathrm{g}}}
$$

and introducing this in Eq. 39.6, we find, for the drift velocity of a heavy ion in a light gas,

$$
v_{d}=\sqrt{\frac{\mathrm{eE} g}{\mathrm{~m}_{\mathrm{r}}}}
$$

A note on "mean free paths" is in order here. When the "probability" of collision $P_{c}$ is measured by a monoenergetic ion beam method, and the angle scattering $I\left(x_{0}\right)$ is also measured, $P_{C}$ can be "corrected" to the momentum transfer collision probability $P_{\mathrm{m}}$ by

$$
P_{m}=P_{c} \frac{\int I\left(x_{0}\right)\left(1-\cos x_{o}\right) d \cos x_{o}}{\int I\left(x_{0}\right) d \cos x_{o}}
$$

and then

$$
\ell_{c}=1 / \mathrm{p}_{\mathrm{o}} \mathrm{P}_{\mathrm{m}}
$$

where $\mathrm{p}_{\mathrm{O}}$ is the pressure in millimeters reduced to $0^{\circ} \mathrm{C}$. The observed scattering angles $\chi_{0}$ can be corrected to the center of gravity angle $\chi_{g}$ by Eq. 38.7, and if these angles are used in Eqs. 41.4 and 41.5, one obtains $\ell_{g}<\ell_{c}$. Neither of these is the Maxwellian mean free path $\ell_{M}$, defined as the mean free path of the particles in a gas at a temperature $T_{g}$. If the mean free path is independent of velocity, $\ell_{M}=l_{g} / \sqrt{2}$.

42. Light ions. The limit of a very light ion in a heavy gas is also known, as this has been treated in Secs. 31 and 35 for electrons. Applying this to the case of a constant 
mean free path with negligible thermal motion of the gas molecules, we know that the distribution function is of the form given by Druyvesteyn

$$
\mathrm{f}=\mathrm{A} \mathrm{e}^{-\mathrm{w}}, \quad \mathrm{w}=\frac{3 \mathrm{~m}}{\mathrm{M}+\mathrm{m}}\left(\frac{\mathrm{u}}{\mathrm{e} \mathrm{E} \ell_{\mathrm{c}}}\right)^{2}
$$

From this distribution, one can calculate the drift velocity by Eq. 31.9:

$$
\mathrm{v}_{\mathrm{d}}=\frac{\sqrt{\pi}}{3 \Gamma\left(\frac{3}{4}\right)}\left(\frac{3 \mathrm{~m}}{\mathrm{M}+\mathrm{m}}\right)^{\frac{1}{4}} \sqrt{\frac{2 \mathrm{eE \ell _{c }}}{\mathrm{m}}}=\gamma\left(\frac{\mathrm{m}}{\mathrm{M}+\mathrm{m}}\right)^{\frac{1}{4}} \sqrt{\frac{\mathrm{eE \ell _{ \textrm {g } }}}{\mathrm{m}_{\mathrm{r}}}}
$$

where account has been taken of the persistence of velocity factor to convert mean free paths in the laboratory system to the mean free paths in the center of gravity system. In similar fashion, one can calculate the mean energy

$$
\overline{\mathrm{m}} \mathrm{v}^{2}=\sqrt{\frac{M+\mathrm{m}}{6 \mathrm{~m}}} \pi \mathrm{eEl_{ \textrm {c } }}
$$

These expressions are not unlike those found above for heavy ions and one may therefore expect to be able to write for all mass ratios

$$
\begin{aligned}
& \mathrm{v}_{\mathrm{d}}=\gamma\left(\frac{\mathrm{m}}{\mathrm{M}+\mathrm{m}}\right)^{\frac{1}{4}} \sqrt{\frac{\pi \mathrm{eEl}}{\mathrm{m}_{\mathrm{r}}}} \\
& \mathrm{m}_{\mathrm{r}}^{\overline{2}}=\mathrm{M}\left(\mathrm{v}^{2}+\alpha \mathrm{v}_{\mathrm{d}}^{2}\right) \\
& \mathscr{E}=\beta \frac{3 \mathrm{~m}}{\mathrm{M}+\mathrm{m}} \frac{\mathrm{m} \mathrm{v}_{\mathrm{x}}^{2}-M \overline{\mathrm{v}_{\mathrm{x}}^{2}}}{(\mathrm{M}+\mathrm{m}) \overline{\mathrm{v}_{\mathrm{x}}^{2}}}
\end{aligned}
$$

where the parameters $\alpha, \beta$, and $\gamma$ are slowly varying functions of the mass ratio. We have assumed that $\nu_{\mathrm{g} 2}=\nu_{\mathrm{g} 1}$ in the ellipticity formula and we have introduced the factor $\frac{m}{M+m}$ quite arbitrarily because it goes to zero and one at the two limits, as it should. 
The work of Wannier (30) gives the values of $\alpha, \beta$, and $\gamma$ for $\mathrm{m}=\mathrm{M}$ so that we can construct the following table:

\begin{tabular}{|c|c|c|c|}
\hline $\mathrm{m} / \mathrm{M}$ & 0 & 1 & $\infty$ \\
\hline$\alpha$ & 1.0607 & 0.789 & 1 \\
$\beta$ & & 0.731 & 1 \\
$\gamma$ & 0.8973 & 0.9643 & 1 \\
\hline
\end{tabular}

d. Polarizable Molecules

43. Force between ion and molecule. A molecule in an electric field E acquires a dipole moment

$$
\overrightarrow{\mathrm{P}}=\alpha \overrightarrow{\mathrm{E}}
$$

where $\alpha$ is the polarizability of the molecule, and this polarizability of the molecule results in a dielectric coefficient $\mathrm{K}$ for the gas given by

$$
\frac{\alpha \mathrm{n}_{\mathrm{g}}}{\epsilon_{\mathrm{O}}}=3 \frac{\epsilon-\epsilon_{\mathrm{O}}}{\epsilon+2 \epsilon_{\mathrm{O}}} \approx \mathrm{K}-1
$$

where $\mathrm{n}_{\mathrm{g}}$ is the number of molecules per unit volume. The electric field of an ion will also polarize a molecule and the induced dipole moment of the molecule will produce an electric field at the ion, resulting in an attractive force between ion and molecule

$$
F=\left(\frac{e}{4 \pi \epsilon_{o}}\right)^{2} \frac{2 \alpha}{r^{5}}
$$

whose potential is

$$
\Phi=-\frac{\alpha}{2}\left(\frac{\mathrm{e}}{4 \pi \epsilon_{\mathrm{o}} \mathrm{r}^{2}}\right)^{2}
$$


This polarization force is exactly the type considered in Secs. 39 and 40 and is the major long-range force between ion and molecule. The same force, of course, exists between an electron and a molecule. In the case of an electron at energy below the ionization energy, the electron's wavelength is comparable to the diameter of the atom, and therefore wave mechanics must be used, and diffraction effects occur, resulting in a rather complicated dependence of the cross section on the electron's velocity. The wavelengths of ions are very much smaller than those of electrons and therefore classical mechanics can be used. Vogt and Wannier (32) have shown that quantum mechanics gives substantially the same orbits as classical mechanics in this case. The classical orbits are soluble in terms of elliptic functions (33). If $u$ is the relative kinetic energy of the particles at infinity and $b$ the impact parameter, the orbits fall into two classes, according to the value of $b^{4} u$. If $b^{4} u>2 \alpha\left(e / 4 \pi \epsilon_{0}\right)^{2}$, the orbit is open, resembling a hyperbola. If $\mathrm{b}^{4} \mathrm{u}<2 \alpha\left(\mathrm{e} / 4 \pi \epsilon_{\mathrm{o}}\right)^{2}$, the orbit goes through the origin. In the singular case, $\mathrm{b}^{4} \mathrm{u}=2 \alpha\left(\mathrm{e} / 4 \pi \epsilon_{\mathrm{o}}\right)^{2}$, the orbit is a spiral asymptotic to a circle of radius $b / \sqrt{2}$. The central orbits certainly correspond to collisions and the frequency of such collisions is given by

$$
\nu=\mathrm{n}_{\mathrm{g}} \pi \mathrm{b}^{2} \mathrm{v}=\frac{\mathrm{en}}{2 \epsilon_{\mathrm{o}}} \sqrt{\frac{\alpha}{\mathrm{m}_{\mathrm{r}}}}=\frac{\mathrm{e}}{2} \sqrt{\frac{\mathrm{n}_{\mathrm{g}}(\mathrm{K}-1)}{\epsilon_{\mathrm{o}} \mathrm{m}_{\mathrm{r}}}}
$$

The open orbits also contribute to the collision frequency in accordance with the scheme of Eqs. 27.5, 27.6, and 27.7 and we have, in the center of gravity system,

$$
\begin{aligned}
& \nu_{\mathrm{g} 0}=\infty \\
& \nu_{\mathrm{g} 1}=1.1052 \nu \\
& \nu_{\mathrm{g} 2}=1.158 \nu
\end{aligned}
$$

Substituting from Eqs. 43.7 and 43.5 in Eq. 39.6 gives, for the mobility,

$$
\mu=0.905 \sqrt{\frac{4 \epsilon_{\mathrm{o}}}{\mathrm{n}_{\mathrm{g} \mathrm{m}_{\mathrm{r}}(\mathrm{K}-1)}}}
$$

In comparing the mobilities at low $\mathrm{E} / \mathrm{p}$ of different ions in the same gas (same value of $\mathrm{K}$ ), the dependence on the $-\frac{1}{2}$ power of the reduced mass is well verified (36). The 
dependence on the dielectric constant $\mathrm{K}$, as measured macroscopically, is qualitatively correct but not exact. The difference can be attributed to both the nonuniformity of the electric field of an ion and to its time variation.

There is an additional effect which occurs when an ion penetrates the wave function of a similar but neutral atom. The atomic electron readily transfers to the ion, leaving the atom ionized. There is no "persistence of velocity" in this transfer so it counts as a full collision in the laboratory system. The theory of this process has been given by Holstein (34) and results in a charge transfer cross section $Q_{t}=\pi r_{t}^{2}$, where the radius $r_{t}$ is determined by

$$
\frac{M V_{o}}{m V}=\alpha r_{t} e^{2 \alpha r_{t}}
$$

Here $1 / \alpha$ is a constant equal to the atomic radius, $V_{0}$ is a potential of the order of 200 volts, and $\mathrm{V}$ is the collision energy. This formula leads to collision transfer radii of over 5 times the normal atomic radius and nearly constant.

Besides the long-range polarization force between ion and molecule, there is a short-range repulsive force because the exclusion principle prevents the electron shells of ion and molecule from overlapping too much. This force increases very rapidly as molecule and ion approach and may be approximated by a rapidly rising potential, as in the collision of rigid spheres. This leads to a constant mean free path; hence for energetic collisions the equations of Sec. 42 are appropriate. Measurements of $\mathrm{P}_{\mathrm{c}}$ for ions (35) indicate that an energy above one electron-volt is necessary for the rigid sphere to begin to be effective in scattering. Ions of near thermal energy should, therefore, always show a constant mean free time.

44. The Langevin theory. The transition from a polarization force to a rigid sphere force was elegantly treated by Langevin (37) and recalculated by Hasse (38). The mobility was worked out as a function of a parameter $\lambda$ given by

$$
\lambda^{2}=\frac{\mathrm{kT}}{-\Phi}{ }_{\mathrm{c}}=\frac{2 \mathrm{kT}}{\alpha}\left(\frac{4 \epsilon_{\mathrm{o}} \mathrm{Q}}{\mathrm{e}}\right)^{2}
$$

where $Q$ is the rigid-sphere cross section, and $\Phi_{C}$ is the potential (Eq. 43.4) of the ion when in contact with the rigid-sphere part of the force. They found the mobility 


$$
\mu=A \sqrt{\frac{4 \pi \epsilon}{\mathrm{n}_{\mathrm{g}} \mathrm{m}_{\mathrm{r}}(\mathrm{K}-1)}}
$$

where $A$ is a function of $\lambda$ that goes to $0.9048 / \sqrt{\pi}$ as $\lambda$ goes to zero, corresponding to Eq. 43.9, and to $\frac{3}{4 \lambda}$ for large $\lambda$, corresponding to Eq. 39. 7. Unfortunately, their calculations were based on the assumption that the ions were in thermal equilibrium with the gas and had a random energy $\frac{3}{2} k \mathrm{~T}$. The Langevin theory can be rescued, until a more correct theory is worked out, by replacing $3 \mathrm{k}$ T by the correct random energy $M\left(v_{d}^{2}+v^{2}\right)$, or, since the thermal velocity of the gas does not matter for either low or high drift velocities, by simply replacing $3 \mathrm{k} T$ by $\mathrm{M} \mathrm{v}_{\mathrm{d}}^{2}$.

Let $\mathrm{a}$ and $\mathrm{c}$ be two constants defined by

$$
\mathrm{a}=\frac{4 \epsilon_{\mathrm{o}} \mathrm{Q}}{\mathrm{e}} \sqrt{\frac{2 \mathrm{M}}{3 \alpha}}
$$

where $\alpha$ is the polarizability and $Q$ the rigid-sphere cross section, and

$$
\begin{aligned}
& \mathrm{c}=\frac{\mathrm{n} g}{2 \epsilon_{\mathrm{o}}} \sqrt{\frac{\alpha \mathrm{m}_{r}}{\pi}} \\
& \frac{\mathrm{c}}{\mathrm{a}}=\mathrm{e} \frac{\mathrm{K}-1}{4 \epsilon_{\mathrm{o}} \mathrm{Q}} \sqrt{\frac{3}{8 \pi} \frac{\mathrm{m}}{\mathrm{M}+\mathrm{m}}}
\end{aligned}
$$

We then have

$$
\begin{aligned}
& v_{d}=\frac{\lambda}{a} \\
& \mu=\frac{A}{c} \\
& E=\frac{c \lambda}{a A}
\end{aligned}
$$

Therefore, $\lambda / \mathrm{A}$ is to be taken as the independent variable, proportional to $\mathrm{E} / \mathrm{p} . \mathrm{A}$ and $\lambda$ are the dependent variables which give the mobility (as before) and the drift velocity. 
The ratio $\lambda / A$ has been computed from Hasse's figures and is given below. A peculiarity of the Langevin theory is that the mobility goes through a maximum during the transition. This has been observed by Hershey (39).

Table I

\begin{tabular}{|l|l|l|l|l|l|l|l|l|}
\hline$\lambda / \mathrm{A}=\mathrm{aE} / \mathrm{c}$ & $\mathrm{A}=\mathrm{c} \mu$ & $\lambda=\mathrm{av}_{\mathrm{d}}$ & $\lambda / \mathrm{A}=\mathrm{aE} / \mathrm{c}$ & $\mathrm{A}=\mathrm{c} \mu$ & $\lambda=\mathrm{av}_{\mathrm{d}}$ & $\lambda / \mathrm{A}=\mathrm{aE} / \mathrm{c}$ & $\mathrm{A}=\mathrm{c} \mu$ & $\lambda=\mathrm{av}_{\mathrm{d}}$ \\
\hline 0.00 & 0.5105 & 0.0 & 3.034 & 0.4614 & 1.4 & 10.773 & 0.2599 & 2.8 \\
0.1822 & 0.5488 & 0.1 & 3.408 & 0.4402 & 1.5 & 11.531 & 0.2515 & 2.9 \\
0.3541 & 0.5648 & 0.2 & 3.809 & 0.4201 & 1.6 & 12.315 & 0.2436 & 3.0 \\
0.5212 & 0.5756 & 0.3 & 4.238 & 0.4011 & 1.7 & 13.124 & 0.2362 & 3.1 \\
0.6854 & 0.5836 & 0.4 & 4.695 & 0.3834 & 1.8 & 13.962 & 0.2292 & 3.2 \\
0.8495 & 0.5886 & 0.5 & 5.180 & 0.3668 & 1.9 & 14.825 & 0.2226 & 3.3 \\
1.0163 & 0.5904 & 0.6 & 5.692 & 0.3514 & 2.0 & 15.719 & 0.2163 & 3.4 \\
1.1909 & 0.5878 & 0.7 & 6.232 & 0.3370 & 2.1 & 16.635 & 0.2104 & 3.5 \\
1.3803 & 0.5796 & 0.8 & 6.798 & 0.3236 & 2.2 & 17.578 & 0.2048 & 3.6 \\
1.5895 & 0.5662 & 0.9 & 7.393 & 0.3111 & 2.3 & 18.556 & 0.1994 & 3.7 \\
1.824 & 0.5483 & 1.0 & 8.016 & 0.2994 & 2.4 & 19.547 & 0.1944 & 3.8 \\
2.084 & 0.5277 & 1.1 & 8.662 & 0.2886 & 2.5 & 20.580 & 0.1895 & 3.9 \\
2.373 & 0.5057 & 1.2 & 9.339 & 0.2784 & 2.6 & 21.633 & 0.1849 & 4.0 \\
2.689 & 0.4834 & 1.3 & 10.041 & 0.2689 & 2.7 & & & \\
\hline
\end{tabular}




\section{THE FOKKER-PLANCK EQUATION (40)}

a. Flow in Velocity Space

45. Relation of the Fokker-Planck equation to the transport equation. The interactions of electrons with other electrons and with ions through their Coulomb fields are of very much longer range than even the inverse fifth-power polarization fields, which lead to a constant mean free path; this introduces the difficulty that collisions are no longer distinct, separate events, because an electron interacts with many other electrons and ions simultaneously. A less serious difficulty which enters in considering electronelectron interactions is that the two interacting distribution functions $F$ and $f$ are now identical, so that the Boltzmann equation is quadratic in $f$, and in order to solve it, it must somehow be linearized. Both of these difficulties are alleviated by considering the Fokker-Planck equation instead of the Boltzmann equation. This equation is readily derived from the transport equation 37.3. The quantity $X^{\prime}-X$ is expanded in powers of the small changes $\Delta \vec{v}$ of the velocity occurring in a collision,

$$
X^{\prime}=X+\Delta v_{i} \frac{\partial X}{\partial v_{i}}+\frac{1}{2} \Delta v_{i} \Delta v_{j} \frac{\partial^{2} X}{\partial v_{i} \partial v_{j}}+\ldots
$$

Introducing this in the transport equation without field terms,

$$
\int X \frac{\partial f}{\partial t} d^{3} v=\iiint\left(X^{\prime}-X\right) F(V) f(v) \rho d^{2} \Omega d^{3} v d^{3} v
$$

and integrating by parts, yields

$$
\begin{aligned}
& \int \mathrm{X} \frac{\partial \mathrm{f}}{\partial \mathrm{t}} \mathrm{d}^{3} \mathrm{v} \\
& \quad=\iint\left[-\mathrm{X} \frac{\partial}{\partial \mathrm{v}_{i}} \int \Delta \mathrm{v}_{i} f \rho \mathrm{d}^{2} \Omega+\frac{1}{2} \mathrm{X} \frac{\partial^{2}}{\partial \mathrm{v}_{i} \partial \mathrm{v}_{j}} \int \Delta \mathrm{v}_{i} \Delta \mathrm{v}_{j} f \rho \mathrm{d}^{2} \Omega \ldots\right] F d^{3} \mathrm{vd} \mathrm{d}^{3} \mathrm{v}
\end{aligned}
$$

We introduce a vector quantity

$$
\vec{\gamma}=\iint \mathrm{F} \Delta \overrightarrow{\mathrm{v}} \rho \mathrm{d}^{2} \Omega \mathrm{d}^{3} \mathrm{~V}
$$


and a tensor quantity

$$
\gamma_{i j}=\iint F \Delta v_{i} \Delta v_{j} \rho d^{2} \Omega d^{3} V
$$

We can then define a flow vector in velocity space by

$$
\Gamma_{i}=\gamma_{i} f-\frac{1}{2} \frac{\partial \gamma_{i j} f}{\partial v_{j}}+\ldots
$$

The vector $m \vec{\gamma}$, having the dimensions of a force, enters the equations like a friction force, and has been termed the dynamical friction. In general, it is directed oppositely to the velocity of an electron and is the force which will bring the velocity of a fast electron, shot into a swarm of electrons and ions, down to the average velocity of the swarm. The quantity $\gamma_{i j}$ enters the equations like a diffusion coefficient but in velocity space instead of in configuration space. It is, the refore, called the diffusion-in-velocity tensor. The term $\mathrm{m} \gamma_{i j}$ has the dimensions of power. The dynamical friction will reduce the average velocity of any electron in a swarm down to the drift velocity of the swarm. The diffusion in velocity will maintain the mean squared velocity of any electron near the average for the swarm and thus maintain equipartition. The higher terms in Eq. 45.6 have not been given names because they are not absolutely essential to the rmal equilibrium, and also because random interactions tend to make them vanish. We note that they exist but will not calculate them.

Introducing $\vec{\Gamma}$ into Eq. 45.3 gives

$$
\int x \frac{\partial f}{\partial t} d^{3} v=\int-x \operatorname{div} \vec{\Gamma} d^{3} v
$$

and, as this equation must hold for all functions $X$,

$$
\frac{\partial f}{\partial t}=-\operatorname{div} \vec{\Gamma}
$$

This is the Fokker-Planck equation. As it is derived here, the Fokker-Planck equation is a series approximation for the Boltzmann equation in powers of the deflections $\Delta \vec{v}$ that occur in a collision. It will converge best when collisions result in many small deflections, that is, for long-range forces, and, therefore, is appropriate for Coulomb forces. A different derivation based on the Langevin equation will be given in Sec. d. 
46. Conservation theorems. Because $\partial f / \partial t$ is given by the Fokker-Planck equation as the divergence of a flow vector and, as this flow vector vanishes at infinity with $f$, the conservation of particles is automatically assured. This is not automatic, however, for momentum or energy. The rate of change of total momentum and energy follows from the Fokker-Planck equation by multiplying by $\mathrm{mv}$ and $\frac{1}{2} \mathrm{mv}^{2}$ and integrating by parts.

$$
\frac{\partial}{\partial t} \mathrm{~m} \overrightarrow{\vec{v}}=\int \mathrm{m} \vec{\gamma} \mathrm{fd} \mathrm{d}^{3} \mathrm{v}
$$

and

$$
\frac{\partial \bar{u}}{\partial t}=\int m\left(\vec{\gamma} \cdot \vec{v}+\frac{1}{2} \gamma\right) \mathrm{fd}^{3} \mathrm{v}
$$

where $\gamma=\Sigma \gamma_{\mathrm{ii}}$ is the trace of the diffusion-in-velocity tensor. If the distribution $\mathrm{F}$ has spherical symmetry, the vector $\vec{\gamma}$ is radial, and if $\mathrm{f}$ also has spherical symmetry, the integral in Eq. 46.1 vanishes. In the theory of Brownian motion the dynamical friction is taken from Stokes' law

$$
\vec{\gamma}=-\beta \vec{v}
$$

It is then assumed that the diffusion-in-velocity coefficient is both constant and a scalar, in order for Eq. 46.2 to vanish. It then follows that

$$
\gamma=2 \beta \overline{\mathrm{v}}=6 \beta \mathrm{k} \mathrm{T} / \mathrm{m}
$$

This simple procedure is quite unsatisfactory in the present case where both $\gamma$ and $\gamma_{i j}$ turn out to be complicated functions of the velocity.

\section{b. Rutherford Scattering}

47. Mean deflections. Let us now consider two interacting particles and the coordinate system $r, s, t$ attached to the center of gravity. The deflection of the particle of mass $\mathrm{m}$ in collision with a particle of mass M (Fig. 13) are given by 


$$
\left.\begin{array}{rl}
\Delta v_{r} & =\frac{M c}{M+m}\left(1-\cos x_{g}\right) \\
\Delta v_{s} & =\frac{M c}{M+m} \sin x_{g} \cos \psi \\
\Delta v_{t} & =\frac{M c}{M+m} \sin x_{g} \sin \psi
\end{array}\right\}
$$

Squaring, we obtain

$$
\left.\begin{array}{l}
\left(\Delta v_{r}\right)^{2}=\left(\frac{M c}{M+m}\right)^{2}\left(1-\cos x_{g}\right)^{2} \\
\left(\Delta v_{s}\right)^{2}=\left(\frac{M c}{M+m}\right)^{2} \sin ^{2} x_{g} \cos ^{2} \psi \\
\left(\Delta v_{t}\right)^{2}=\left(\frac{M c}{M+m}\right)^{2} \sin ^{2} x_{g} \sin ^{2} \psi
\end{array}\right\}
$$

These quantities must be integrated over scattering angles and over the distribution $F$ to give $\gamma_{i}$ and $\gamma_{i j}$. Let us introduce the symbols $\delta_{i}$ and $\delta_{i j}$ for the results of the integration over scattering angles; and use the definitions 27.6 and 27.7 changed to the center of gravity system, and also use $n_{+}$to indicate charged scatters. Then

$$
\left.\begin{array}{l}
\delta_{\mathrm{r}}=\int \Delta \mathrm{v}_{\mathrm{r}} \rho \mathrm{d}^{2} \Omega=-\frac{\mathrm{Mc}}{\mathrm{M}+\mathrm{m}} \frac{\nu_{\mathrm{g} 1}}{\mathrm{n}_{+}} \\
\delta_{\mathrm{rr}}=2\left(\frac{\mathrm{Mc}}{\mathrm{M}+\mathrm{m}}\right)^{2} \frac{3 \nu_{\mathrm{g} 1}-\nu_{\mathrm{g} 2}}{3 \mathrm{n}_{+}} \\
\delta_{\mathrm{ss}}=\delta_{\mathrm{tt}}=\left(\frac{\mathrm{Mc}}{\mathrm{M}+\mathrm{m}}\right)^{2} \frac{\nu_{\mathrm{g} 2}}{3 \mathrm{n}_{+}}
\end{array}\right\}
$$

and all other $\delta$ 's vanish because $\rho$ does not depend on $\psi$. Thus the vector $\vec{\delta}$ is directed towards the center of gravity and the tensor $\delta_{i j}$ has the structure

$$
\delta_{i j}=\left(\frac{M c}{M+m}\right)^{2} \frac{1}{3 n_{+}}\left\|\begin{array}{cccc}
6 \nu_{g 1}-2 \nu_{g 2} & 0 & 0 \\
0 & \nu_{g 2} & 0 \\
0 & 0 & \nu_{g 2}
\end{array}\right\|
$$

This combination can be represented, as in Fig. 14, by an arrow and an ellipsoid of revolution. For isotropic scattering the ellipsoid is elongated in the direction of the 


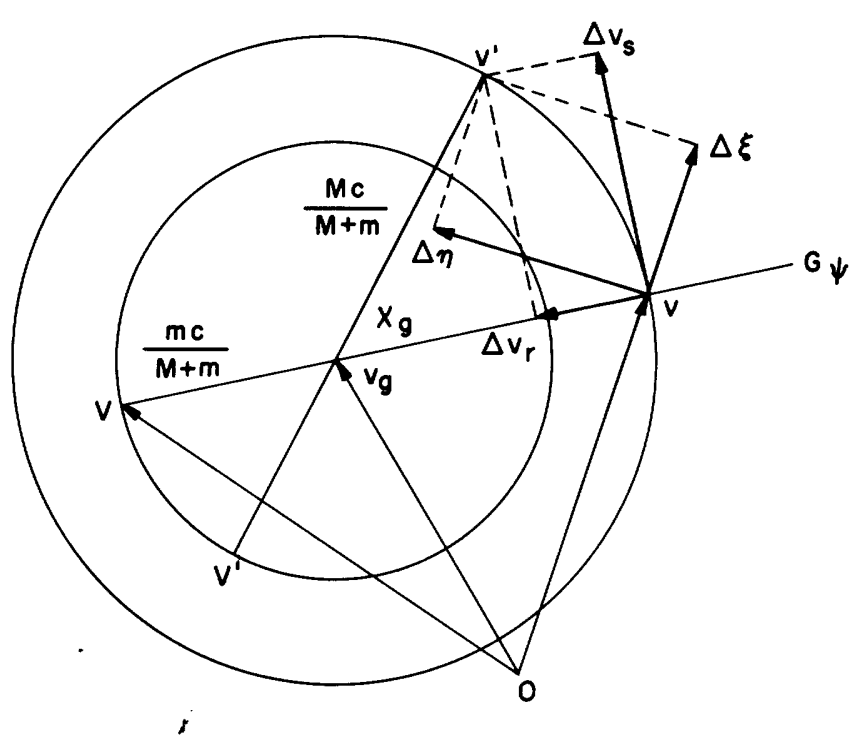

Fig. 13. Components of $\Delta \vec{v}$ in center of gravity coordinates $r, s, t$, and in laboratory coordinates $\xi, \eta, \zeta$.

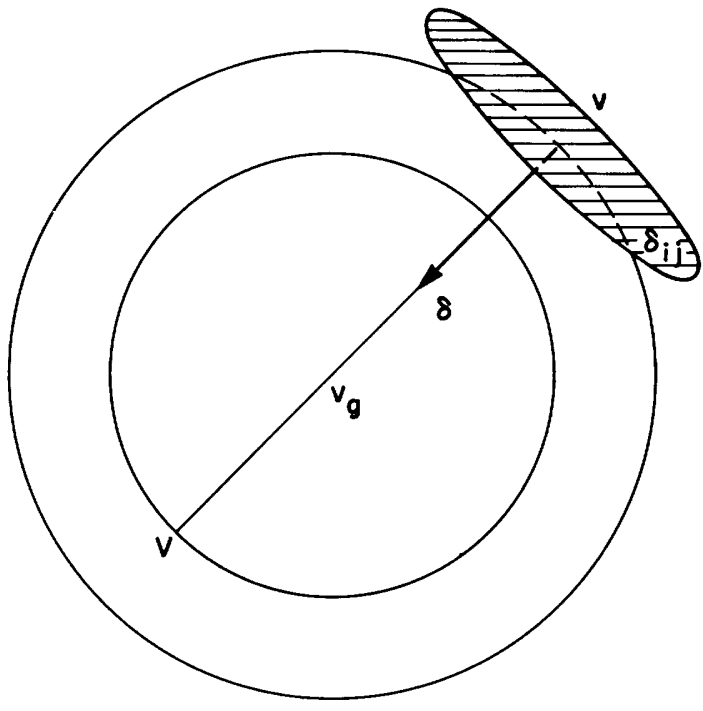

Fig. 14. Dynamical friction vector and diffusion tensor in relative coordinates.

relative velocity, but if scattering is largely in the forward direction, that is, small angle scattering, the ellipsoid is very much flattened in the direction of the relative velocity. This situation comes from the factor $\left(1-\cos x_{g}\right)^{2}$ in Eq. 47.2 which strongly weights backward scattering. At an angle as large as $30^{\circ}$, this factor has only 4 per cent of its value at $180^{\circ}$. The Fokker-Planck equation converges rapidly only for small angle scattering and, as $\left(1-\cos \chi_{g}\right)^{2}$ weights backward scattering very strongly, one may expect that $\delta_{\mathrm{rr}}$ will appear in higher-order terms of the Fokker-Planck equation than $\delta_{\mathrm{ss}}, \delta_{\mathrm{tt}}$ and $\delta_{\mathrm{r}}$. As we shall use only the first and second-order terms of the FokkerPlanck equation, we shall find it necessary eventually to neglect all terms in $\delta_{\mathrm{rr}}$

It is noted that the four nonvanishing $\delta$ 's contain only two $\nu_{\mathrm{g}}^{\prime} \mathrm{s}$. There are, therefore, invariant relations between the $\delta$ 's which do not depend on the scattering law. It is the se relations which result in relatively simple expressions (Eq. 52.6) for the flow vector $\vec{\Gamma}$. In particular the trace of the tensor $\delta_{i j}$ is a scalar

$$
\delta=2\left(\frac{\mathrm{Mc}}{\mathrm{M}+\mathrm{m}}\right)^{2} \frac{\nu_{\mathrm{g} 1}}{\mathrm{n}_{+}}
$$

and is simply related to the vector $\delta_{\mathbf{r}}$.

48. Shielded Rutherford scattering. If the scattering particles of the last section

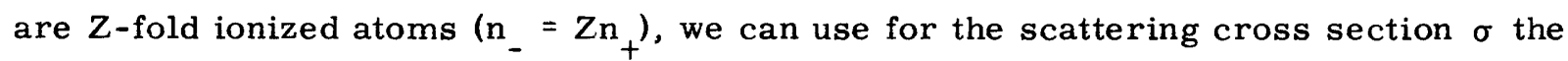
Rutherford scattering formula 


$$
\sigma=\left[\frac{\mathrm{Ze}^{2}}{4 \pi \epsilon_{\mathrm{o}} \mathrm{m}_{\mathrm{r}} \mathrm{c}^{2}\left(1-\cos \chi_{\mathrm{g}}\right)}\right]^{2}
$$

However, the integrals for both $\nu_{g 1}$ and $\nu_{g 2}$ diverge. Only the combination in $\delta_{\mathrm{rr}}$ converges, yielding

$$
\delta_{\mathrm{rr}}=\frac{\pi \mathrm{K}}{\mathrm{c}}, \quad \mathrm{K}=\left(\frac{\mathrm{Ze}^{2}}{2 \pi \epsilon_{\mathrm{o}} \mathrm{m}}\right)^{2}
$$

The other integrals diverge logarithmically for small scattering angles. Coulomb forces are too long ranged to consider the interaction to be simply between two particles at all distances. This problem was considered by Chandrasekhar (41) in computing the time of relaxation of stellar systems and led him to cut off the Rutherford cross section (Eq. 48.1) for an impact parameter equal to the average distance between stars. Spitzer (42) considers the same problem for electrons and ions and cuts off the Rutherford cross section at a scattering angle $x_{D}$ for which the distance of closest approach is equal to the Debye distance $\ell_{D}$. The theory of Debye and Hückel shows that the average potential of an ion is

$$
V=\frac{Z \text { e }}{4 \pi \epsilon_{0} r} \exp \left(-r / \ell_{D}\right), \quad \ell_{D}^{2}=\frac{\epsilon_{0} k T}{n_{-} e^{2}}
$$

and, therefore, the average orbit of an electron going by an ion will be the classical orbit of a particle in such a field. This problem has not been solved, but cutting off the Rutherford cross section at the angle $x_{D}$ is an approximation thereto. The use of an average potential is probably correct in calculating mean deflections, and its application to mean squared deflections is then required for consistency. In the interaction of an electron with an ion, the ion is hardly disturbed, and, therefore, will retain its electron cloud which shields its potential. The use of the Debye sphere in electron-electron interactions (43) is somewhat more difficult to justify. We shall assume the interaction (48.3) above to be good in both cases. Fortunately, the cutoff only appears in a logarithmic term and the difference between the assumptions of Chandrasekhar and Spitzer is not as large as one might fear. The angle $x_{D}$ is given by

$$
1-\cos x_{D}=\frac{2}{1+q^{2}}
$$


where

$$
\mathrm{q}=\frac{\mathrm{m}_{\mathrm{r}} \mathrm{c}^{2}}{2} \frac{4 \pi \epsilon_{\mathrm{o}} \mathrm{L}_{\mathrm{D}}}{\mathrm{z} \mathrm{e}^{2}}
$$

in which $q$ is a variable, as it contains $c$, but, as $q$ appears only in a logarithm, it is convenient to treat it as a constant, replacing $\mathrm{m}_{\mathrm{r}} \mathrm{c}^{2}$ by its average value $3 \mathrm{k} T$. Then

$$
\overline{\mathrm{q}}=6 \pi \mathrm{n}_{-} \ell_{\mathrm{D}}^{3} / \mathrm{Z}=9 \mathrm{~N}_{+} / 2
$$

where $\mathrm{N}_{+}$is the number of positive ions in the Debye sphere. It then follows from Eqs. 47.6 and 47.3 that

$$
\delta=2 \pi \mathrm{K} \mathrm{L} / \mathrm{c}
$$

and that

$$
\delta_{\mathrm{r}}=-\frac{\mathrm{M}+\mathrm{m}}{\mathrm{M}} \frac{\pi \mathrm{KL}}{\mathrm{c}^{2}}
$$

where

$$
L=\ln \sqrt{1+q^{2}}
$$

Values of $L$ for different values of $\log n_{-}\left(\frac{e}{k T}\right)^{3}$, where the argument is in $(\text { volt }-\mathrm{cm})^{-3}$ are given below.

Table II

\begin{tabular}{|c|c|}
\hline $\log \mathrm{n}_{-}(\mathrm{e} / \mathrm{k} \mathrm{T})^{3}$ & $\mathrm{~L}=1 \mathrm{n} \sqrt{1+\mathrm{q}^{2}}$ \\
\hline 10 & 11.256 \\
11 & 10.104 \\
12 & 8.953 \\
13 & 7.802 \\
14 & 6.650 \\
15 & 5.500 \\
16 & 4.348 \\
17 & 3.192 \\
18 & 2.053 \\
19 & 1.528 \\
20 & 1.528 \\
\hline
\end{tabular}


It is seen that, for most discharges, $\mathrm{L}$ is of the order 10 so that $\delta_{\mathrm{rr}}$ is actually small compared with $\delta$ and the ellipsoid of Fig. 14 is flattened, as indicated. The number $\mathrm{N}_{+}$ varies as $1 / \sqrt{\bar{n}_{-}}$and is down to one for $\mathrm{n}_{-}=3 \times 10^{18}(\mathrm{k} \mathrm{T} / \mathrm{e})^{3} \mathrm{~cm}^{-3}$. As it seems unreasonable to extend the concept of Debye to less than one particle, the shielding at high concentrations has been set at $\mathrm{N}_{+}=1$.

The deflection tensor $\delta_{i j}$ now has the form

$$
\delta_{i j}=\frac{\pi \mathrm{K}}{\mathrm{c}}\left\|\begin{array}{ccc}
1 & 0 & 0 \\
0 & \mathrm{~L}-\frac{1}{2} & 0 \\
0 & 0 & \mathrm{~L}-\frac{1}{2}
\end{array}\right\|
$$

The approximation of replacing $\mathrm{c}$ by $\overline{\mathrm{c}}$ in Eq. 48.5 makes $\mathrm{L}$ a constant, and it will be so treated in the manipulations that follow. However, this approximation is only valid if $\mathrm{L}$ is large, and it leads to a contradiction (Eq. 53.8 does not then lead to zero flow when $f$ is a Maxwell distribution) unless we also neglect the non- $L$ terms in the tensor 48.9. This amounts to neglecting $\delta_{\mathrm{rr}}$ and equivalent terms. We, therefore, write

$$
\delta_{i j}=\frac{\pi \mathrm{K}}{\mathrm{c}}\left\|\begin{array}{ccc}
0 & 0 & 0 \\
0 & \mathrm{~L} & 0 \\
0 & 0 & \mathrm{~L}
\end{array}\right\|
$$

The retained terms correspond to the large forward scattering, rendered finite by the Debye shielding. The neglected terms correspond to the much smaller large-angle scattering, and do not contribute to the trace of the tensor.

49. Rosenbluth potentials for a single scattering. The scattering has been integrated over a sphere to obtain the vector $\delta_{r}$ and tensor $\delta_{i j}$. These must now be integrated over the distribution in velocity space according to Eqs. 45.4 and 45.5 in order to obtain the dynamical friction and diffusion-in-velocity. However, vectors, and to a higher degree tensors, are awkward to integrate. M. Rosenbluth (44) has shown that $\delta_{\mathbf{r}}$ and $\delta_{i j}$ can be obtained from two "potentials"

$$
\begin{aligned}
& \mathrm{g}_{1}=\pi \mathrm{KL}|\mathrm{c}| \\
& \mathrm{h}_{1}=\frac{\pi \mathrm{KL}}{|\mathrm{c}|}
\end{aligned}
$$

where the subscript 1 indicates that they are the potentials for a single scattering (the subscript will be dropped when they are integrated over the distribution function); and 
$\mathrm{h}_{1}$ is an ordinary Coulomb potential, except that it is taken in velocity space, and corresponds to the fact that the dynamical friction $\vec{\delta}$ varies inversely as the square of the relative velocity

$$
\vec{\delta}=-\frac{M+m}{M} \vec{\nabla} h_{1}
$$

The mass factor is close to one for ion-electron scattering, but it is two for electronelectron scattering. The new potential is $g_{1}$, and it is easy to show that

$$
\vec{\nabla} \mathrm{g}_{1}=\pi \mathrm{KL} \overrightarrow{\mathrm{c}} /|\mathrm{c}|
$$

and

$$
\delta_{i j}=\frac{\partial^{2} g_{1}}{\partial c_{i} \partial c_{j}}
$$

where the components $c_{i}$ and $c_{j}$ are taken in Cartesian coordinates. The relation between the two Rosenbluth potentials is found by taking the trace of Eq. 49.5

$$
\nabla^{2} g_{1}=-2 h_{1}
$$

50. Coulomb's law in velocity space. The relation between dynamical friction in a plasma and Coulomb's law allows us to apply immediately all the results of potential theory. Consider, for instance, scattering particles whose distribution in velocity space is spherical. Then we know that the dynamical friction on a test particle in this distribution can be attributed entirely to particles whose speed is less than the speed of the test particle and these latter can be considered concentrated at the center of the spherical distribution.

$(\alpha)$ Let us apply this to the conductivity of a completely ionized plasma. Let us take axes moving with the electron drift and assume that the electrons have a spherical distribution

$$
f=n_{-}\left(\frac{m}{2 \pi k T}\right)^{3 / 2} e^{-m v^{2} / 2 k T}
$$

and let us suppose that the ions have a small drift velocity $v_{d}$ relative to the electrons and have very little random velocity about this drift velocity. Then the dynamical friction acting on the ions is attributable entirely to the 


$$
\mathrm{n}_{\mathrm{d}}=\frac{2 \mathrm{n}_{-}}{3 \sqrt{2 \pi}}\left(\frac{\mathrm{mv}_{\mathrm{d}}^{2}}{\mathrm{kT}}\right)^{3 / 2}
$$

electrons whose velocity is less than $\mathrm{v}_{\mathrm{d}}$, and the dynamical friction is equal to

$$
M \vec{\Gamma}=-M \frac{M+m}{m} \frac{\pi K L}{v_{d}^{3}} \vec{v}_{d} n_{+} n_{d}=-\frac{Z L v_{d}}{3 m_{r}}\left(\frac{m}{2 \pi k T}\right)^{3 / 2}\left(\frac{n_{-} e^{2}}{\epsilon_{0}}\right)^{2}
$$

This dynamical friction must be balanced by the applied electric force

$$
\mathrm{M} \vec{\Gamma}+\mathrm{n}_{-} \mathrm{eE}=0
$$

from which we obtain the conductivity

$$
\sigma=\frac{\mathrm{n}_{-} \mathrm{ev}_{\mathrm{d}}}{\mathrm{E}}=\frac{3 \mathrm{~m} r}{\mathrm{ZL}}\left(\frac{\epsilon_{\mathrm{o}}}{\mathrm{e}}\right)^{2}\left(\frac{2 \pi \mathrm{kT}}{\mathrm{m}}\right)^{3 / 2}
$$

This formula turns out to be too small (45) by a factor 1.9747 mainly because of the neglect of diffusion-in-velocity, which acts to reduce the dynamical friction, but it illustrates the application of potential theory.

$(\beta)$ Consider now a fast electron, such that the dynamical friction acting on it is due to all the other electrons and is $-2 \pi \mathrm{n} \mathrm{KL} / \mathrm{v}^{2}$. In the presence of an external electric field $\overrightarrow{\mathrm{E}}$, the total force on such an electron is

$$
\overrightarrow{\mathrm{F}}=-\mathrm{e} \overrightarrow{\mathrm{E}}-2 \pi \mathrm{mKL} \mathrm{nv} / \mathrm{v}^{3}
$$

This is the formula for the resultant of a uniform field plus a point charge, and the flow lines of such a field are given by

$$
v^{2}=\frac{2 v_{b}^{2}}{1+\cos \theta}+\frac{C}{\sin ^{2} \theta}
$$

where $\mathrm{C}$ is an arbitrary constant, and the product

$$
\mathrm{E} \frac{\mathrm{m}_{\mathrm{r}} \mathrm{v}_{\mathrm{b}}^{2}}{2 \mathrm{e}}=2 \pi \mathrm{L} \frac{\mathrm{en}^{\frac{2}{3}}}{4 \pi \epsilon_{\mathrm{o}}} \frac{\mathrm{en}^{\frac{1}{3}}}{4 \pi \epsilon_{\mathrm{o}}}=13 \times 10^{-18} \mathrm{n} \text { volt }{ }^{2} / \text { meter }
$$

is seen to be related to the product of the electric field and potential at the interelectronic 


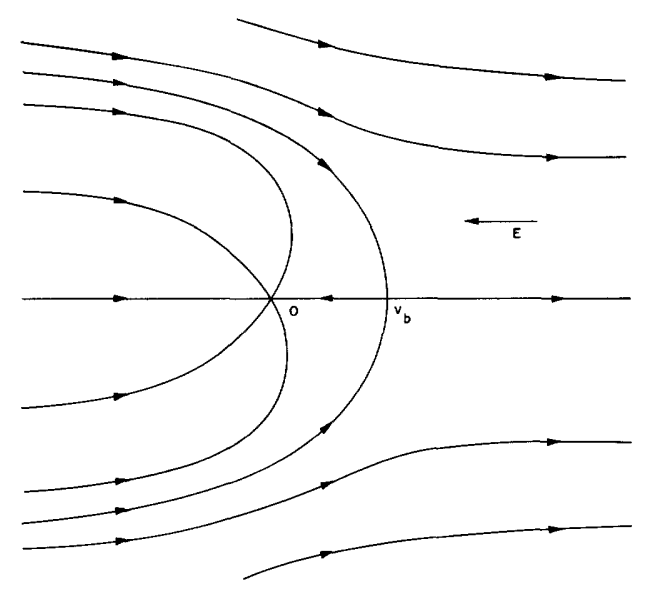

Fig. 15. Flow in velocity space attributable to external field and dynamical friction.

distance $\mathrm{n}^{-1 / 3}$. The flow lines are of two kinds.

For negative $\mathrm{C}$, the flow lines go through the origin. For positive $\mathrm{C}$, they go to infinity at both ends, the singular line separating these two categories being given by $\mathrm{C}=0$. If we neglect the effects of diffusion-in-velocity, which give rise to transitions between lines, it is seen that electrons whose velocity is such that $\mathrm{C}$ is negative tend to stay with the electron distribution, whereas electrons of faster velocity for which $\mathrm{C}$ is positive will be accelerated indefinitely by the applied field and tend to escape. The escape velocity is $v_{b}$ for an electron moving in the direction of the field.

51. The potentials of a scattering field. The two Rosenbluth potentials

$$
\begin{aligned}
& g(v)=\int \pi K L c F d^{3} V \\
& h(v)=\int \frac{\pi K L}{c} F d^{3} V
\end{aligned}
$$

are obtained by integrating the single scattering potentials over the velocity field of the scattering particles, and it will be assumed that their velocity distribution is expanded in Legendre polynomials in $\mu=\cos \Theta$ :

$$
F(\vec{V})=\sum F^{\ell}(V) P_{\ell}(\mu)
$$

It is then necessary, in order to perform the integrations for the potentials $\mathrm{g}(\mathrm{v})$ and 
$\mathrm{h}(\mathrm{v})$, to expand $|\mathrm{c}|$ and $1 /|\mathrm{c}|$ in Legendre polynomials in $\mathrm{x}$, the cosine of the angle between the velocities $\mathrm{v}$ and $\mathrm{V}$ of the colliding particles:

$$
\begin{aligned}
& |c|= \begin{cases}v \sum\left[\frac{(\mathrm{V} / \mathrm{v})^{2}}{2 \ell+3}-\frac{1}{2 \ell-1}\right]\left(\frac{\mathrm{v}}{\mathrm{v}}\right)^{\ell} \mathrm{P}_{\ell}(\mathrm{x}) & \mathrm{v}<\mathrm{v} \\
\mathrm{v} \sum\left[\frac{(\mathrm{v} / \mathrm{V})^{2}}{2 \ell+3}-\frac{1}{2 \ell-1}\right]\left(\frac{\mathrm{v}}{\mathrm{V}}\right)^{\ell} \mathrm{P}_{\ell}(\mathrm{x}) & \mathrm{v}>\mathrm{v}\end{cases} \\
& \frac{1}{|\mathrm{c}|}= \begin{cases}\mathrm{v} \sum\left(\frac{\mathrm{v}}{\mathrm{v}}\right)^{\ell} \mathrm{P}_{\ell}(\mathrm{x}) & \mathrm{v}<\mathrm{v} \\
\mathrm{v} \sum\left(\frac{\mathrm{v}}{\mathrm{V}}\right)^{\ell} \mathrm{P}_{\ell}(\mathrm{x}) & \mathrm{v}>\mathrm{v}\end{cases}
\end{aligned}
$$

The necessary integrals are all of the forms

$$
\begin{array}{ll}
I_{j}^{\ell}=4 \pi v^{-j} \int_{0}^{V} F^{\ell} V^{2+j} d V & j \geq 0 \\
I_{-j}^{\ell}=4 \pi v^{j} \int_{v}^{\infty} F^{\ell} v^{2-j} d V \quad j>0
\end{array}
$$

it being noted that $I_{O}^{O}(v)$ gives the concentration of particles of speed less than $v$, and $I_{1}^{1}$ is related to the current density. These integrals satisfy the following differential relation:

$$
\left(v \frac{\partial}{\partial v} \pm j\right) I_{ \pm j}^{\ell}= \pm 4 \pi v^{3} F^{\ell}
$$

Substituting in Eqs. 51.1 and 51.2, we find the two potentials

$$
\mathrm{g}=\pi \mathrm{KLV} \sum\left[\frac{\mathrm{I}_{\ell+2}^{\ell}+\mathrm{I}_{-1-\ell}^{\ell}}{2 \ell+3}-\frac{\mathrm{I}_{\ell}^{\ell}+\mathrm{I}_{1-\ell}^{\ell}}{2 \ell-1}\right] \frac{\mathrm{P}_{\ell}(\mu)}{2 \ell+1}
$$




$$
\mathrm{h}=\frac{\pi \mathrm{KL}}{\mathrm{v}} \sum\left[\mathrm{I}_{\ell}^{\ell}+\mathrm{I}_{1-\ell}^{\ell}\right] \frac{\mathrm{P}_{\ell}(\mu)}{2 \ell+1}
$$

52. Flow in velocity space. One obtains the diffusion-in-velocity $\gamma_{i j}$ and the dynamical friction $\vec{\gamma}$ by applying the gradient operator to the potentials, but, as these are desired in polar coordinates, we shall first give the suitable modification of Eq. 49.5.

$$
\begin{aligned}
& \gamma_{\mathrm{vv}}=\frac{\partial^{2} \mathrm{~g}}{\partial \mathrm{v}^{2}} \\
& \gamma_{\mathrm{v} \theta}=\frac{\sin \theta}{\mathrm{v}}\left(\frac{1}{\mathrm{v}} \frac{\partial \mathrm{g}}{\partial \mu}-\frac{\partial^{2} \mathrm{~g}}{\partial \mathrm{v} \mu}\right) \\
& \gamma_{\theta \theta}=\frac{1}{\mathrm{v}} \frac{\partial \mathrm{g}}{\partial \mathrm{v}}-\frac{\mu}{\mathrm{v}^{2}} \frac{\partial \mathrm{g}}{\partial \mu}+\frac{1-\mu^{2}}{\mathrm{v}^{2}} \frac{\partial^{2} \mathrm{~g}}{\partial \mu^{2}} \\
& \gamma_{\phi \phi}=\frac{1}{\mathrm{v}} \frac{\partial \mathrm{g}}{\partial \mathrm{v}}-\frac{\mu}{\mathrm{v}} \frac{\partial \mathrm{g}}{\partial \mu} \\
& \gamma_{\mathrm{v} \phi}=\gamma_{\theta \phi}=0
\end{aligned}
$$

It is readily verified that the trace $\gamma$ of this tensor is the Laplacian of g; hence

$$
\gamma=\frac{1}{v^{2}} \frac{\partial}{\partial v} v^{2} \frac{\partial g}{\partial v}+\frac{1}{v^{2}} \frac{\partial}{\partial \mu}\left(1-\mu^{2}\right) \frac{\partial g}{\partial \mu}=-2 h
$$

Similarly,

$$
\left.\begin{array}{l}
\gamma_{\mathrm{v}}=-\frac{\mathrm{M}+\mathrm{m}}{\mathrm{M}} \frac{\partial \mathrm{h}}{\partial \mathrm{v}} \\
\gamma_{\theta}=-\frac{\mathrm{M}+\mathrm{m}}{\mathrm{Mv}} \frac{\partial \mathrm{h}}{\partial \theta} \\
\gamma_{\phi}=0
\end{array}\right\}
$$

Substitution for $g$ and $h$ from Eqs. 51.8 and 51.9 gives the components of $\gamma_{i j}$ and $\gamma_{i}$ in terms of the integrals $I_{+j}^{\ell}$. These components can then be substituted in Eq. 45.6 to obtain the flow $\Gamma_{i}$ in velocity space. This expression can be written, using only secondorder terms, 


$$
\begin{aligned}
\Gamma_{i} & =\left(\gamma_{i}-\frac{1}{2} \sum_{j} \frac{\partial \gamma_{i j}}{\partial v_{j}}\right) f-\frac{1}{2} \sum_{j} \gamma_{i j} \frac{\partial f}{\partial v_{j}} \\
& =\gamma_{i}^{\prime} f-\frac{1}{2} \sum_{j} \gamma_{i j} \frac{\partial f}{\partial v_{j}}
\end{aligned}
$$

It now follows from the relation (Eq. 49.6) between $g$ and $h$ that, were it not for the mass factor $1+\mathrm{m} / \mathrm{M}$ in Eq. 49.3, the two terms in parentheses would exactly cancel, and this is a consequence of the form of the diffusion tensor 48.10. Including the mass factor, we find

$$
\vec{\gamma}^{\prime}=\frac{\mathrm{m}}{\mathrm{M}+\mathrm{m}} \vec{\gamma}
$$

The "effective" dynamical friction $\vec{\gamma}^{\prime}$ is, in the case of electron-electron scattering, reduced to half the "true" value $\vec{\gamma}$, and, in the case of electron-ion scattering, is reduced several thousandfold. This cancellation expresses the fact that electrons can lose only a very small fraction of their energy in a collision with an ion, although they lose one half, on the average, in colliding with a stationary electron. Thus, $\vec{\gamma}^{\prime}$ is the effective dynamical friction for energy transfer.

We can now write

$$
\begin{aligned}
& \Gamma_{\mathrm{v}}=\gamma_{\mathrm{v}}^{\prime} \mathrm{f}-\frac{1}{2}\left(\gamma_{\mathrm{vv}} \frac{\partial \mathrm{f}}{\partial \mathrm{v}}+\gamma_{\mathrm{v} \theta} \frac{\partial \mathrm{f}}{\mathrm{v} \partial \theta}\right) \\
& \Gamma_{\theta}=\gamma_{\theta}^{\prime} \mathrm{f}-\frac{1}{2}\left(\gamma_{\theta \mathrm{v}} \frac{\partial \mathrm{f}}{\partial \mathrm{v}}+\gamma_{\theta \theta} \frac{\partial \mathrm{f}}{\mathrm{v} \partial \theta}\right) \\
& \Gamma_{\phi}=0
\end{aligned}
$$

\section{c. Maxwellian Distribution of Scatterers}

53. Reduction of the flow vector. We shall first assume that the expansion 51.3 for $F$ requires only the first two terms. Substitution of Eq. 51.8 in Eq. 52.1 then yields the following components for the diffusion-in-velocity:

$$
\gamma_{\mathrm{vv}}=\frac{2 \pi \mathrm{KL}}{\mathrm{v}}\left[\frac{\mathrm{I}_{2}^{\mathrm{O}}+\mathrm{I}_{-1}^{\mathrm{O}}}{3}+\frac{\mathrm{I}_{3}^{1}+\mathrm{I}_{-2}^{1}}{5} \cos \theta\right]
$$




$$
\begin{aligned}
& \gamma_{\theta \theta}=\gamma_{\phi \phi}=\frac{\pi \mathrm{KL}}{\mathrm{v}}\left[\mathrm{I}_{\mathrm{o}}^{\mathrm{O}}+\frac{2 \mathrm{I}_{-1}^{\mathrm{O}}-\mathrm{I}_{2}^{\mathrm{O}}}{3}+\frac{5 \mathrm{I}_{1}^{1}-3 \mathrm{I}_{3}^{1}+2 \mathrm{I}_{-2}^{1}}{15} \cos \theta\right] \\
& \gamma_{\mathrm{v} \theta}=\frac{\pi \mathrm{KL}}{\mathrm{v}} \frac{3 \mathrm{I}_{3}^{1}-5 \mathrm{I}_{1}^{1}-2 \mathrm{I}_{-2}^{1}}{15} \sin \theta=\frac{\partial \gamma_{\theta \theta}}{\partial \theta} \\
& \gamma=\frac{2 \pi \mathrm{KL}}{\mathrm{v}}\left[\mathrm{I}_{\mathrm{O}}^{\mathrm{O}}+\mathrm{I}_{-1}^{\mathrm{O}}+\frac{\mathrm{I}_{1}^{1}+\mathrm{I}_{-2}^{1}}{3} \cos \theta\right]
\end{aligned}
$$

Substituting Eq. 51.9 in Eq. 52.3 we obtain the dynamical friction:

$$
\begin{aligned}
& \gamma_{\mathrm{v}}=-\frac{\mathrm{M}+\mathrm{m}}{\mathrm{M}} \frac{\pi \mathrm{KL}}{\mathrm{v}^{2}}\left[\mathrm{I}_{\mathrm{o}}^{\mathrm{O}}+\frac{2 \mathrm{I}_{1}^{1}-\mathrm{I}_{-2}^{1}}{3} \cos \theta\right] \\
& \gamma_{\theta}=-\frac{\mathrm{M}+\mathrm{m}}{\mathrm{M}} \frac{\pi \mathrm{KL}}{\mathrm{v}^{2}} \frac{\mathrm{I}_{1}^{1}+\mathrm{I}_{-2}^{1}}{3} \sin \theta
\end{aligned}
$$

These expressions are further reduced by a choice of axes moving with the center of gravity of the scattering particles. Then, $F^{1}=0, I_{j}^{1}=0, \gamma_{v \theta}=\gamma_{\theta}=0$, and the flow equations reduce to

$$
\begin{aligned}
& \Gamma_{\mathrm{v}}=\gamma_{\mathrm{v}}^{\mathrm{f}} \mathrm{f}-\frac{1}{2} \gamma_{\mathrm{vv}} \frac{\partial \mathrm{f}}{\partial \mathrm{v}} \\
& \Gamma_{\theta}=-\frac{1}{2} \gamma_{\theta \theta} \frac{\partial \mathrm{f}}{\mathrm{v} \partial \theta}
\end{aligned}
$$

Furthermore, if the scattering distribution is Maxwellian,

$$
\begin{aligned}
& \mathrm{F}=\mathrm{n}_{+}\left(\frac{\mathrm{M}}{2 \pi \mathrm{kT}}\right)^{3 / 2} \mathrm{e}^{-\mathrm{MV}^{2} / 2 \mathrm{kT}} \\
& \mathrm{I}_{\mathrm{O}}^{\mathrm{O}}=\mathrm{n} \in \mathrm{rf} \sqrt{\frac{\mathrm{Mv}^{2}}{2 \mathrm{kT}}}-\frac{4 \pi k T}{\mathrm{M}} \mathrm{vF}
\end{aligned}
$$

The vector and tensor components are

$$
\gamma_{\mathrm{V}}^{\prime}=-\pi \mathrm{KL} \frac{\mathrm{m}}{\mathrm{M}} \mathrm{I}_{\mathrm{O}}^{\mathrm{O}}
$$




$$
\left.\begin{array}{l}
\gamma_{\mathrm{vv}}=-\frac{2 \mathrm{kT}}{\mathrm{mv}} \gamma_{\mathrm{v}}^{\prime} \\
\gamma_{\theta \theta}=\frac{\pi \mathrm{KL}}{\mathrm{v}}\left[\left(1-\frac{\mathrm{kT}}{\mathrm{Mv}^{2}}\right) \mathrm{I}_{\mathrm{O}}^{\mathrm{O}}+\frac{4 \pi \mathrm{kT}}{\mathrm{M}} \mathrm{v} \mathrm{F}\right]
\end{array}\right\}
$$

and the flow equations are

$$
\begin{aligned}
& \Gamma_{\mathrm{v}}=\gamma_{\mathrm{v}}^{\prime}\left(\mathrm{f}+\mathrm{kT} \frac{\partial \mathrm{f}}{\partial \mathrm{u}}\right) \\
& \Gamma_{\theta}=-\frac{\gamma_{\theta \theta}}{2 \mathrm{v}} \frac{\partial \mathrm{f}}{\partial \theta}
\end{aligned}
$$

It is evident that the flow in velocity space vanishes if, and only if, the scattered particles have a Maxwellian distribution at the same temperature as the scatterers. This is indeed as it must be and shows that the approximations of Sec. 48 are consistent. If one treats $\mathrm{L}$ as a constant but does not neglect the non- $\mathrm{L}$ terms in 48.9 this relation is not found.

54. Electron interactions. Integrating Eq. 53.8 over a sphere in velocity space gives the gain, caused by interactions,

$$
G=-\frac{m}{M} 4 \pi^{2} K L I_{o}\left(f^{o}+k T \frac{\partial f^{o}}{\partial u}\right)
$$

and this gives the rate at which a nonequilibrium distribution tends towards equilibrium. If $f$ is Maxwellian at a temperature above $T$, the gain is everywhere negative and the distribution contracts. Reciprocally, if $\mathrm{f}$ is too cold, it expands. Ions are relatively inefficient in thermalizing an electron distribution because of the factor $\mathrm{m} / \mathrm{M}$. Of course, if we are considering the interactions of electrons, $I_{0}$ itself is an integral over $f$ and

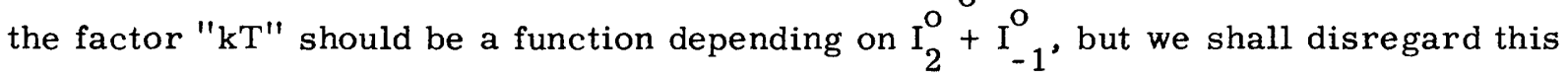
error in the following discussion. However, in solving the gain equation we must remember that $\mathrm{kT}$ is itself an unknown, representing the mean energy of the unknown $\mathrm{f}^{\circ}$.

We would like to study the transition in electron distribution functions from those given in Sec. 35 as electron-electron interactions become important. To do this we merely add the gain (Eq. 54.1) due to interactions to the gain (Eq. 34.2) due to the field and collisions:

$$
-\frac{G}{4 \pi}=\left(\frac{\nu_{c} u_{c} v^{3}}{3}+\pi K L I_{o} k T\right) \frac{\partial f}{\partial u}+\left(\frac{m}{M} \nu_{c} v^{3}+\pi K L I_{o}\right) f
$$

and use this in the gain equation 34.2. Expanding $I_{0}$ in powers of $v$, 


$$
I_{0}=\frac{n}{3} \sqrt{\frac{2}{\pi}}\left(\frac{m}{k T}\right)^{3 / 2} v^{3}\left(1-\frac{3 m v^{2}}{10 \mathrm{kT}} \ldots\right)
$$

and comparing this with the gain due to the electric field, we see that, for slow electrons, the interaction first becomes dominant at a concentration

$$
\mathrm{n}_{-}=\sqrt{\frac{\mathrm{mkT}}{2 \pi}}\left(\frac{2 \pi \epsilon_{\mathrm{o}}}{\mathrm{e}^{2}}\right)^{2} \frac{\nu_{\mathrm{c}} \mathrm{u}_{\mathrm{c}}}{\mathrm{L}}
$$

or at a degree of ionization

$$
\frac{\mathrm{n}_{-}}{\mathrm{n}_{\mathrm{g}}}=\frac{\mathrm{u}_{\mathrm{c}}}{\mathrm{e}} \sqrt{\frac{\mathrm{kT}}{\mathrm{e}}} \frac{\nu_{\mathrm{c}}}{10^{8} \mathrm{p}_{\mathrm{o}}} \frac{1.3}{10^{3} \mathrm{~L}}
$$

Each factor in this product is generally less than one, particularly the last one which is about $10^{-4}$. For degrees of ionization above $10^{-4}$, the distribution functions will look more Maxwellian than those derived in Sec. 35.

Even though the electron concentration may considerably exceed the limit set by Eq. 54.4, there are two circumstances that keep the electron interactions from modifying the distribution functions too severely. Electron interactions modify neither their number nor their total energy. The plasma balance equation

$$
\bar{\nu}_{\mathrm{i}}=\mathrm{D} / \Lambda^{2}
$$

and the energy balance equation

$$
\text { ne } \mu E^{2}=\int_{v_{x}}^{\infty} \nu_{x} u f 4 \pi v^{2} d v+\int_{0}^{\infty} \frac{3 m}{M} \nu_{c} u f 4 \pi v^{2} d v
$$

do not contain the interaction terms explicitly.

As the electron concentration becomes high and the two interaction terms in the gain become large, the temperature becomes indeterminate. We are then approaching thermal equilibrium and the temperature is determined by Saha's equation

$$
\frac{n_{a}}{n_{+}{ }^{n}-}=\frac{g_{a}}{2 g_{i}} \frac{h^{3}}{(2 \pi m k T)^{3 / 2}} e^{e V_{i} / k T}
$$

where $n_{a}$ is the number of atoms remaining neutral, $g_{a}$ and $g_{i}$ the statistical weights of 
the ground states of atom and ion.

55. Electron-ion interactions. If the scattering particles are ions of mass $M \gg m$, all terms in $1 / M$ in Eqs. 53.6 and 53.7, are negligible, leaving only

$$
\begin{aligned}
& \gamma_{\theta \theta}=\frac{\pi \mathrm{KL}}{\mathrm{v}} \mathrm{I}_{\mathrm{o}}=\frac{\pi \mathrm{L} \mathrm{Kn}+}{\mathrm{v}} \\
& \Gamma_{\theta}=\frac{\pi \mathrm{KLn}+}{2 \mathrm{v}^{2}} \frac{\partial \mathrm{f}}{\partial \theta}=-\frac{\pi \mathrm{L} \mathrm{Kn}+}{2 \mathrm{v}^{2}} \sin \theta \mathrm{f}^{1}
\end{aligned}
$$

all other terms being smaller than this one in the ratio $\mathrm{m} / \mathrm{M}$. If we project on the Z-axis,

$$
\Gamma_{\mathrm{z}}=-\Gamma_{\theta} \sin \theta=\frac{\pi \mathrm{KL} \mathrm{n}+}{2 \mathrm{v}^{2}} \mathrm{f}^{1} \sin ^{2} \theta
$$

and the total dynamical friction on the electrons is

$$
\mathrm{m} \iint \Gamma_{\mathrm{z}} \mathrm{d}^{2} \Omega \mathrm{v}^{2} \mathrm{dv}=\frac{4 \pi^{2}}{3} \mathrm{mKL} \mathrm{n}_{+} \int \mathrm{f}^{1} \mathrm{dv}
$$

which must, in the absence of other collisions, be equal to the applied force $n_{-} e E$. The current is given by Eq. 19.7 as

$$
J=e \int \frac{4 \pi}{3} f^{1} v^{3} d v=\sigma_{i} E
$$

from which one obtains the conductivity $\sigma_{i}$

$$
\sigma_{i}=\frac{n_{-} e^{2}}{\pi m K L n_{+}} \frac{\int f^{1} v^{3} d v}{\int f^{1} d v}=\frac{\pi m}{Z L}\left(\frac{2 \epsilon_{o}}{e}\right)^{2} \frac{\int f^{1} v^{3} d v}{\int f^{1} d v}
$$

Spitzer shows that the distribution $\mathrm{f}^{1}$ is given approximately by

$$
f^{1}=A v^{2} e^{-m v^{2} / 2 k T}
$$

whence the conductivity is

$$
\sigma_{i}=\frac{m}{Z L}\left(\frac{4 \pi \epsilon_{o}}{e}\right)^{2}\left(\frac{2 \mathrm{kT}}{\pi \mathrm{m}}\right)^{\frac{3}{2}} \times 1.1632
$$


where the numerical factor was obtained by Spitzer by numerical integration of a distribution function slightly different from Eq. 55.7. The conductivity of a completely ionized plasma is independent of the concentration $n_{-}$because the number of resisting elements $\mathrm{n}_{+}$is proportional to the number of conducting elements $\mathrm{n}_{-}$. However, if the atoms are more than singly ionized, the conductivity is reduced. The conductivity increases as the three-halves power of the temperature because $c$ times the Rutherford scattering cross section varies inversely as the cube of the relative velocity.

56. Conductivity of a partially ionized plasma. If the electrons collide with neutral gas as well as being deflected by the ions, the collision frequencies add and, therefore, the resistivities approximately add

$$
\frac{1}{\sigma}=\frac{1}{\sigma_{i}}+\frac{1}{n_{-} e_{\mu}}
$$

We are interested in the transition from "mobility" to "conductivity", that is, in the ratio

$$
x=\frac{n_{-} e \mu}{\sigma_{i}}=c w_{i}^{3 / 2} \frac{n_{+}}{n_{g}}
$$

where

$$
\mathrm{w}_{\mathrm{i}}=\mathrm{eV}_{\mathrm{i}} / \mathrm{kT}
$$

and

$$
\mathrm{c}=\mathrm{L}\left(\mu \mathrm{n}_{\mathrm{g}}\right) \sqrt{\frac{\mathrm{m}}{\mathrm{e}}}\left(\frac{\pi}{2 \mathrm{~V}_{\mathrm{i}}}\right)^{3 / 2}\left(\frac{\mathrm{Ze}}{4 \pi \epsilon_{\mathrm{o}}}\right)^{2}=\frac{\mathrm{z}^{2} \mathrm{~L}}{4.2} \frac{\left(\mu \mathrm{p}_{\mathrm{o}}\right)}{\mathrm{v}_{\mathrm{i}}^{3 / 2}}
$$

In the expression for $c, \mu$ is in $\mathrm{m}^{2} / \mathrm{sec}$ volt and $\mathrm{p}$ in $\mathrm{mm}, \mathrm{c}$ is a number of the order of 2 , so the value of $n_{g} / n_{+}$for the transition depends mainly on $w_{i}$. As this is generally greater than 5 , the conductivity transition takes place when the plasma is less than 5 per cent ionized, a much higher per cent than for the electron interaction transition. We may, therefore, be justified in using Saha's equation in this section.

The meaning of $\mathrm{n}_{\mathrm{g}}$ is somewhat ambiguous. In principle, it is the concentration of neutral atoms, $\mathrm{n}_{\mathrm{g}}=\mathrm{n}_{\mathrm{a}}$, but if the ions are not stripped, they have remaining electron shells and these contribute to the scattering. If they scatter equally with neutral atoms, $n_{g}=n_{o}$, the original concentration of gas atoms. We shall adopt this last assumption. Saha's equation (Eq. 54. 8) can be written 


$$
\begin{aligned}
& \frac{n_{a}}{n_{+} n_{-}}=\frac{d}{Z n_{0}} w_{i}^{3 / 2} e^{w_{i}} \\
& b=\frac{g_{a}}{2 g_{i}} \frac{{z n_{o} h^{3}}_{\left(2 \pi \mathrm{m} \mathrm{e}_{i}\right)^{3 / 2}}}{g_{i}} \frac{g_{a}}{v_{i}^{3 / 2}} \frac{p}{17 \times 10^{4}}
\end{aligned}
$$

where $\mathrm{p}$ is in $\mathrm{mm}$. Therefore, $\mathscr{D}$ is always very small.

Eliminating $n_{+}$between Eqs. 56.2 and 56.5 gives an equation for the ratio $X$ in terms of the temperature:

$$
\begin{aligned}
& \lrcorner e^{w_{i}}=w_{i}^{3 / 2} \frac{c^{2}}{x^{2}} \pm \frac{c}{x} \\
& \text { (+ for stripped ions } n_{g}=n_{a}, \quad-\text { for normal ions } n_{g}=n_{o} \text { ) }
\end{aligned}
$$

One can usually neglect the last term, and the conductivity ratio is given by

$$
x^{2}=\frac{c^{2}}{s} w_{i}^{3 / 2} e^{-w_{i}}
$$

\section{d. Stochastic Processes}

57. The Fokker-Planck equation. The derivation of the Fokker-Planck equation given in Sec. 45 is not the conventional one. Following Chandrasekhar (46), let $W\left(\vec{v}^{\prime}, \Delta v\right)$ be the probability that an electron at $\vec{v}^{\prime}$ changes its velocity by $\Delta v$ in a time $\Delta$ t. Then

$$
f(\vec{v}, t+\Delta t)=\int f\left(\vec{v}^{\prime}, t\right) W\left(\vec{v}^{\prime}, \Delta \vec{v}\right) d^{3} \Delta v
$$

Expand both sides in Taylor series:

$$
\begin{aligned}
& f(\vec{v}, t+\Delta t)=f+\frac{\partial f}{\partial t} \Delta t+\ldots \\
& f\left(\vec{v}^{\prime}, t\right) W\left(\vec{v}^{\prime}, \Delta \vec{v}\right)=f(\vec{v}, t) W(\vec{v}, \Delta \vec{v})-\frac{\partial(f W)}{\partial v_{i}} \Delta v_{i}+\frac{1}{2} \frac{\partial^{2}(f W)}{\partial v_{i} \partial v_{j}} \Delta v_{i} \Delta v_{j} \ldots
\end{aligned}
$$

Substituting in Eq. 57.1, and introducing the notation,

$$
\int W d^{3} \Delta v=1
$$




$$
\begin{aligned}
& \int \mathrm{W} \Delta \overrightarrow{\mathrm{v}} \mathrm{d}^{3} \Delta \mathrm{v}=\vec{\gamma} \Delta \mathrm{t} \\
& \int \mathrm{W} \Delta \mathrm{v}_{i} \Delta \mathrm{v}_{j} \mathrm{~d}^{3} \Delta \mathrm{v}=\gamma_{i j} \Delta \mathrm{t}
\end{aligned}
$$

we obtain

$$
\frac{\partial f}{\partial t}=-\operatorname{div} \vec{\gamma} f+\frac{1}{2} \frac{\partial^{2}}{\partial v_{i} \partial v_{j}} \gamma_{i j} f+\ldots
$$

which is formally identical to Eq. 45.8.

58. The Langevin equation. The similarity between Eq. 45.8 and Eq. 57.7 is, however, purely formal. The $\gamma^{\prime}$ s have different meanings in the two equations.

The Langevin equation for an electron is

$$
\frac{d \vec{v}}{d t}=\vec{A}(t)=-e \vec{E}(t) / m
$$

where $\vec{E}(t)$ is the microfield acting on an electron because of its rapidly varying ionic environment. $\vec{A}(t)$ has an average value

$$
\overline{\vec{A}(t)}=\vec{\gamma}
$$

and a correlation function

$$
\overline{A_{i}(t+s) A_{j}(t)}=\psi_{i j}(s)
$$

where the bar represents an assembly average.

Let us assume that

$$
\int_{0}^{\infty} \psi_{i j}(s) d s=\gamma_{i j}
$$

From the Langevin equation,

$$
\Delta \vec{v}=\int_{0}^{\Delta t} \vec{A} d t
$$


and taking averages,

$$
\overrightarrow{\Delta \vec{v}}=\vec{\gamma} \Delta \mathrm{t}
$$

which is the same as Eq. 57.5. Similarly (47),

$$
\begin{aligned}
& \Delta v_{i} \Delta v_{j}=\int_{0}^{\Delta t} \int_{0}^{\Delta t} A_{i}(t) A_{j}(u) d t d u \\
& \overline{\Delta v_{i} \Delta v_{j}}=\int_{0}^{\Delta t} \int_{0}^{\Delta t} \psi_{i j}(s) d t d s=\gamma_{i j} \Delta t
\end{aligned}
$$

provided $\Delta t \gg \tau$, where $\tau$ is the fluctuation time of $\vec{A}(t)$ or the mean width of the correlation function $\psi_{i j}(s)$. This is the same as Eq. 57.6.

The higher-order correlations will generally be finite when integrated once, and, therefore, products such as $\overline{\Delta v_{i} \Delta v_{j} \Delta v_{k}}$ will be proportional to $(\Delta t)^{2}$ or higher powers. It follows then that, if $\Delta t$ is very small (but it must not go to zero), all terms after the first two in the Fokker-Planck equation are also very small, and the two-term equation we have used is correct.

The present derivation is not applicable when $\Delta t \rightarrow 0$, for if $\Delta t \ll \tau, \psi($ s) in Eq. 58.8 is substantially constant and

$$
\overline{\Delta v_{i} \Delta v_{j}} \approx \gamma_{i j}(\Delta t)^{2} / \tau
$$

Even the second-order term in the Fokker-Planck equation then vanishes (48).

The times $\Delta t$ and $\tau$ are readily interpreted in the electron interaction problem. $\Delta \mathrm{t}$ is the time to produce $\Delta \mathrm{v}$ and is therefore the interaction time or the transit time across a Debye sphere. The fluctuation time $\tau$ is the time to change nearest neighbors. $\tau \ll \Delta t$ if there are many particles in a Debye sphere. The unfavorable situation for Boltzmann theory is the favorable one for stochastic theory. But the coefficients $\gamma_{i j}$ should be derived from the correlation of the microfield, not from a collision integral.

Boltzmann theory is ideally suited to rigid sphere collisions, in which $\Delta t \rightarrow 0$, but $\vec{A}(t) \rightarrow \infty$ so that $\Delta \vec{v}$ is not proportional to $\Delta t$. No terms in the Fokker-Planck equation then vanish. The stochastic function $\vec{A}(t)$ is then a random series of sharp impulses so the fluctuation time $\tau$ also vanishes.

If, as in the theory of Brownian motion, we are considering the motion of a very 
heavy particle in a light gas, so that $\Delta \vec{v}$ for one collision is very small, we can change our meanings of $\Delta t$ and $\Delta \vec{v}$ and let them extend over several collisions. We are then back to the conditions of Eq. 58.8, and stochastic theory can be applied. 
1. R. Jancel and T. Kahan, Nuovo Cimento $\underline{12}, 573$ (1954).

2. H. Alfvén, Cosmical Electrodynamics (Clarendon Press, Oxford, 1950).

3. L. Spitzer, Jr., Astrophys. J. 116, 299 (1952).

4. J. H. Van Vleck, Theory of Electric and Magnetic Susceptibilities (Clarendon Press, Oxford, 1952), p. 100.

5. L. Tonks, Phys. Rev. 97, 1443 (1955).

6. W. H. Bennett, Phys. Rev. $\underline{45}, 890$ (1934).

7. P. Langevin, Compt. rend. Acad. Sci. (Paris) 146, 530 (1908). Several basic papers in this field have been reprinted in Noise and Stochastic Processes, edited by N. Wax (Dover Publications, Inc., New York, 1954).

8. S. Chandrasekhar, Stochastic problems in physics and astronomy, Revs. Modern Phys. 15, 21 (1943).

9. L. Tonks, Phys. Rev. $\underline{51}, 744 ; \underline{52}, 710$ (1937).

10. S. Chandrasekhar, Revs. Modern Phys. $\underline{15}, 1$ (1943).

11. Ibid., Equation (120).

12. A. Guthrie and R. K. Wakerling, The Characteristics of Electrical Discharges in Magnetic Fields (McGraw-Hill Book Company, Inc., New York, 1949), p. 64; A. Simon, Phys. Rev. 98, 317 (1955).

13. P. Debye and E. Huckel, Physik. Z. 24, 190 (1923).

14. W. P. Allis and D. J. Rose, Phys. Rev. $\underline{93}, 84$ (1954).

15. I. B. Bernstein and T. Holstein, Phys. Rev. 94, 1475 (1954).

16. W. Schottky, Physik, A. 25, 635 (1924).

17. A. Simon, loc. cit.

18. B. Lax, W. P. Allis, and S. C. Brown, J. Appl. Phys. 21, 1297 (1950).

19. L. Tonks and I. Langmuir, Phys. Rev. 34,876 (1929).

20. S. Chapman and T. G. Cowling, The Mathematical Theory of Non-uniform Gases (Cambridge University Press, London, 1939).

21. H. Margenau, Phys. Rev. 73, 309 (1948).

22. J. H. Jeans, The Dynamical Theory of Gases (Cambridge University Press, London, 4th edition, 1925).

23. E. Gerjuoy and S. Stein, Phys. Rev. 97, 1671 (1955).

24. A. D. MacDonald and S. C. Brown, Phys. Rev. 76, 1634 (1949).

25. A. D. MacDonald and S. C. Brown, Phys. Rev. 75, 411 (1949); W. P. Allis and S. C. Brown, Phys. Rev. 87, 419 (1952); F. Reder and S. C. Brown, Phys. Rev. 95, 885 (1954); A. D. MacDonald and D. D. Betts, Can. J. Phys. 30, 565 (1952). 
26. J. A. Smidt, Physica $\underline{3}, 543$ (1937).

27. L. M. Hartman, Phys. Rev. 37, 316 (1948).

28. M. J. Druyvesteyn and F. M. Penning, Revs. Modern Phys. 12, 87 (1940).

29. H. Margenau, Phys. Rev. 69, 508 (1946).

30. G. H. Wannier, Phys. Rev. 83, 281 (1951); Bell System Tech. J. 32, 170 (1953).

31. J. C. Maxwell, The Scientific Papers of James Clerk Maxwell, edited by W. D. Niven (Cambridge University Press, London, 1890), vol. 2, p. 36.

32. E. Vogt and G. H. Wannier, Phys. Rev. 95, 1190 (1954).

33. W. D. MacMillan, Theoretical Mechanics (McGraw-Hill Book Company, Inc., 1927), p. 297.

34. T. Holstein, J. Phys. Chem. 56, 832 (1952).

35. C. Ramsauer and O. Beeck, Ann. Physik 87, 1 (1928).

36. J. H. Mitchell and K. E. W. Ridler, Proc. Roy. Soc. (London) A146, 911 (1934).

37. P. Langevin, Ann. Chim. Phys. $\underline{5}, 245$ (1905).

38. H. R. Hasse, Phil. Mag. Ser. 7, 1, 139 (1926).

39. A. V. Hershey, Phys. Rev. 56, 908 (1939).

40. Part V is based largely on a $\mathrm{Ph}$. D. thesis of H. Dreicer (Department of Physics, M.I.T., Jan. 1955), which, in turn, rested largely on the work of L. Spitzer, Jr. and R. Harm [Phys. Rev. $\underline{89}, 977(1953)]$ and of S. Chandrasekhar [Astrophys. J. 97, 255; 263 (1943); $98,54 \overline{(1943)]}$.

41. S. Chandrasekhar, Principles of Stellar Dynamics (University of Chicago Press, Chicago, 1942), p. 58.

42. L. Spitzer, Jr. and R. Härm, Phys. Rev. 89, 977 (1953).

43. D. Pines and D. Bohm, Phys. Rev. $\underline{85}, 341$ (1952).

44. Work done at Los Alamos Scientific Laboratory.

45. H. Maecker, Th. Peters, and H. Schenk, Z. Physik 140, 119 (1955), give experimental values of $\sigma$; thus requiring values for $L$ below any that are given in Table II.

46. S. Chandrasekhar, Revs. Modern Phys. 15, 31 (1943).

47. G. E. Uhlenbeck and L. S. Ornstein, Phys. Rev. $\underline{36}, 827$ (1930).

48. The author is indebted to Dr. R. H. Lyon for this observation. 


\section{Bibliography}

1. H. Alfvén, Cosmical Electrodynamics (Clarendon Press, Oxford, 1950), has a chapter on the motion of charged particles that is similar to part $I$ of this report.

2. S. Chapman and T. G. Cowling, The Mathematical Theory of Non-uniform Gases (Cambridge University Press, London, 1939) deal exhaustively with small departures from thermal equilibrium.

3. T. Kihara, Mathematical theory of electrical discharges in gases, Revs. Modern Phys. 24, 45 (1952); 25, 844 (1953), covers the area of part III of this report.

4. L. Spitzer, Jr. and R. Härm, Phys. Rev. 89, 977 (1953), compute the conductivity of an ionized plasma by the method of part $\mathrm{V}$ of this report.

5. G. H. Wannier, Phys. Rev. 83, 281 (1951), gives the most recent work on ion mobilities.

6. N. Wax, Noise and Stochastic Processes (Dover Publications, Inc., New York, 1954) contains reprints of basic papers by S. Chandrasekhar and G. E. Uhlenbeck.

The following references to papers on electron distribution functions are given in chronological order:

7. M. J. Druyvesteyn, Physica 10, 69 (1930).

8. P. M. Morse, W. P. Allis, and E. S. Lamar, Phys. Rev. 82, 412 (1935).

9. B. Davydov, Physik. Z. Sowjetunion $\underline{8}, 59$ (1935).

10. J. A. Smidt, Physica $\underline{3}, 543$ (1937).

11. L. Tonks and W. P. Allis, Phys. Rev. 52, 710 (1937).

12. T. Holstein, Phys. Rev. 70,367 (1946).

13. H. Margenau, Phys. Rev. 73, 297; 309 (1948).

14. A. D. MacDonald and S. C. Brown, Phys. Rev. $\underline{75}, 411$ (1949).

15. W. P. Allis and S. C. Brown, Phys. Rev. 87, 419 (1952). 